$$
\begin{aligned}
& : 1 \mathrm{~J} \\
& \text { AVgebra \& } \\
& \text { Number } \\
& \text { Theory } \\
& \lrcorner\lrcorner 」\lrcorner \\
& \lrcorner \\
& \text { 」ป } \\
& \lrcorner
\end{aligned}
$$

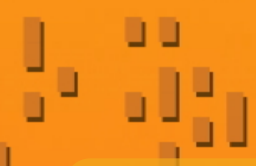
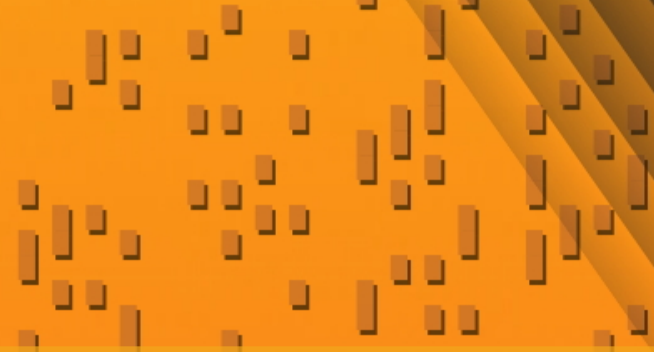

The basic geometry of Witt vectors, I The affine case

James Borger

\lrcorner

\lrcorner

لـ 


\title{
The basic geometry of Witt vectors, I The affine case
}

\author{
James Borger
}

\begin{abstract}
We give a concrete description of the category of étale algebras over the ring of Witt vectors of a given finite length with entries in an arbitrary ring. We do this not only for the classical $p$-typical and big Witt vector functors but also for certain analogues over arbitrary local and global fields. The basic theory of these generalized Witt vectors is developed from the point of view of commuting Frobenius lifts and their universal properties, which is a new approach even for classical Witt vectors. Our larger purpose is to provide the affine foundations for the algebraic geometry of generalized Witt schemes and arithmetic jet spaces, so the basics are developed in some detail, with an eye toward future applications.
\end{abstract}

Introduction

1. Generalized Witt vectors and $\Lambda$-rings 238

2. Grading and truncations 250

3. Principal single-prime case 255

4. General single-prime case 259

5. Multiple-prime case 263

6. Basic affine properties 265

7. Some general descent 268

Language 268

Gluing two objects $\quad 272$

Grothendieck's theorem 275

Gluing and descent of étale algebras 275

8. Ghost descent in the single-prime case 276

9. $W$ and étale morphisms 280

Acknowledgements 284

References $\quad 284$

This work was partly supported by Discovery Project DP0773301, a grant from the Australian Research Council.

MSC2010: 13F35.

Keywords: Witt vector, Witt space, lambda-ring, Frobenius lift, plethory. 


\section{Introduction}

Witt vector functors are certain functors from the category of (commutative) rings to itself. The most common are the $p$-typical Witt vector functors $W$, for each prime number $p$. Given a ring $A$, one traditionally defines $W(A)$ as a set to be $A^{\mathbb{N}}$ and then gives it the unique ring structure which is functorial in $A$ and such that the set maps

$$
\begin{aligned}
W(A) & \stackrel{w}{\longrightarrow} A^{\mathbb{N}} \\
\left(x_{0}, x_{1}, \ldots\right) & \longmapsto\left\langle x_{0}, x_{0}^{p}+p x_{1}, x_{0}^{p^{2}}+p x_{1}^{p}+p^{2} x_{2}, \ldots\right\rangle
\end{aligned}
$$

are ring homomorphisms for all rings $A$, where the target has the ring structure with componentwise operations. For example, we have

$$
\begin{aligned}
\left(x_{0}, x_{1}, \ldots\right)+\left(y_{0}, y_{1}, \ldots\right) & =\left(x_{0}+y_{0}, x_{1}+y_{1}-\sum_{i=1}^{p-1} \frac{1}{p}\left(\begin{array}{l}
p \\
i
\end{array}\right) x_{0}^{i} y_{0}^{p-i}, \ldots\right) \\
\left(x_{0}, x_{1}, \ldots\right) \cdot\left(y_{0}, y_{1}, \ldots\right) & =\left(x_{0} y_{0}, x_{0}^{p} y_{1}+x_{1} y_{0}^{p}+p x_{1} y_{1}, \ldots\right) .
\end{aligned}
$$

Observe that the four polynomials in $x_{0}, y_{0}, x_{1}, y_{1}$ displayed on the right-hand side have integer coefficients, as they must if they are to define operations on $W(A)$ for all rings $A$. Conversely, to prove that the desired functorial ring structure on $W$ exists, it is enough to prove that the polynomials sitting in the higher components have integer coefficients too. This is Witt's theorem.

On the other hand, the polynomials at the component of index $n$ depend only on the variables $x_{0}, y_{0}, \ldots, x_{n}, y_{n}$. This is clear by induction. It follows that the quotient set $A^{[0, n]}=\left\{\left(x_{0}, \ldots, x_{n}\right)\right\}$ of $W(A)=A^{\mathbb{N}}$ is a quotient ring, which we denote by $W_{n}(A)$. (It is traditionally denoted $W_{n+1}(A)$. The shift in indexing is preferable for reasons discussed in 2.5.)

In some cases, the rings $W(A)$ and $W_{n}(A)$ are isomorphic to familiar rings. For example, $W(\mathbb{Z} / p \mathbb{Z})$ is isomorphic to the ring $\mathbb{Z}_{p}$ of $p$-adic integers, and $W_{n}(\mathbb{Z} / p \mathbb{Z})$ is isomorphic to $\mathbb{Z} / p^{n+1} \mathbb{Z}$. If $p$ is invertible in $A$, then $w$ is a bijection and so the Witt vector rings become product rings: $W_{n}(A) \cong A^{[0, n]}$ and $W(A) \cong A^{\mathbb{N}}$. But in most cases, $W(A)$ is not a familiar ring.

While this traditional approach to Witt vectors is adequate for many purposes, it has two shortcomings. The first is that it is not clear how we should think about the affine scheme Spec $W_{n}(A)$ geometrically. Indeed, I am not aware of a truly geometric description of Spec $W_{n}(A)$ in any nontrivial case in the literature. If we want to fully incorporate Witt vectors into arithmetic algebraic geometry (and we do), it is important to have a thorough understanding of their geometry. The main point here and in the companion paper [Borger 2010] is to set up a framework for that. The geometry in this paper is however limited to the basic results in the affine case needed for the general treatment in [Borger 2010]. 
The second shortcoming of the traditional approach is that it does not explain what the defining purpose of Witt vectors is. The answer, at least for this paper, is that they control Frobenius lifts-ring endomorphisms which reduce to the Frobenius map modulo $p$. Here we are following Borger and Wieland [2005, §12.3-4], who in turn followed Joyal [1985a; 1985b]. Motivated by this perspective, we will define Witt vector functors relative to primes in any global or local field. This generality includes not only the $p$-typical functors above but also the so-called big Witt vector functor and less common variants of the $p$-typical ones due to Drinfeld [1976, Proposition 1.1] and to Hazewinkel [1978, (18.6.13)]. It also includes many variants unstudied till now. We will work with these generalized functors throughout the paper. In fact, this will take no more effort once we establish some basic reduction results.

Let us now discuss the contents in more detail.

Section 1 introduces our generalized Witt vectors. Given a Dedekind domain $R$ and a set $E$ of maximal ideals of $R$ with finite residue fields, we will define a functor $W_{R, E}$ from the category $\operatorname{Ring}_{R}$ of $R$-algebras to itself:

$$
W_{R, E}: \operatorname{Ring}_{R} \rightarrow \operatorname{Ring}_{R} .
$$

(In fact, we will work with slightly more general $R$ and $E$.) We call $W_{R, E}$ the $E$ typical Witt vector functor. When $R=\mathbb{Z}$ and $E$ consists of a single maximal ideal $p \mathbb{Z}$, our functor will agree with the $p$-typical Witt vector functor above; when $E$ consists of all maximal ideals of $\mathbb{Z}$, our functor will agree with the big Witt vector functor. The definition of $W_{R, E}$ is in two steps. First we define a functor

$$
W_{R, E}^{\mathrm{fl}}: \operatorname{Ring}_{R}^{\mathrm{fl}} \rightarrow \operatorname{Ring}_{R}^{\mathrm{fl}},
$$

where $\operatorname{Ring}_{R}^{\mathrm{fl}}$ is the full subcategory of $\operatorname{Ring}_{R}$ consisting of $R$-algebras which are $\mathfrak{m}$-torsion free for all ideals $\mathfrak{m} \in E$. We call such algebras $E$-flat. Then we define $W_{R, E}$ to be a certain natural extension of $W_{R, E}^{\mathrm{fl}}$ to all of $\operatorname{Ring}_{R}$.

Let $\mathbb{N}^{(E)}$ denote the commutative monoid $\bigoplus_{E} \mathbb{N}$, where $\mathbb{N}$ is $\{0,1, \ldots\}$ under addition. Given an action of $\mathbb{N}^{(E)}$ on an $R$-algebra $B$, let $\psi_{\mathfrak{m}}$ denote the endomorphism of $B$ given by the $\mathfrak{m}$-th element of the standard basis of $\mathbb{N}^{(E)}$. Let us say that such an action is a $\Lambda_{R, E}$-structure if for each $\mathfrak{m} \in E$, the map $\psi_{\mathfrak{m}}$ reduces to the Frobenius endomorphism $x \mapsto x^{[R: \mathfrak{m}]}$ on $B / \mathfrak{m} B$. Now, for any $R$-algebra $A$, the monoid $\mathbb{N}^{(E)}$ acts on $A^{\mathbb{N}^{(E)}}$ through its translation action on itself in the exponent. When $A$ is $E$-flat, we define $W_{R, E}^{\mathrm{fl}}(A)$ to be the largest of the sub- $R$ algebras $B \subseteq A^{\mathbb{N}^{(E)}}$ having the properties that $B$ is stable under the action of $\mathbb{N}^{(E)}$ and that the induced action on $B$ is a $\Lambda_{R, E}$-structure. It is elementary to check that a maximal such subalgebra $W_{R, E}^{\mathrm{fl}}(A)$ exists. 
This definition can be expressed as a universal property. Let $\operatorname{Ring}_{\Lambda_{R, E}}^{\mathrm{fl}}$ denote the following category: the objects are $E$-flat $R$-algebras equipped with a $\Lambda_{R, E^{-}}$ structure, and the morphisms are $\mathbb{N}^{(E)}$-equivariant $R$-algebra maps. Then $W_{R, E}^{\mathrm{fl}}$, viewed as a functor $\operatorname{Ring}_{R}^{\mathrm{fl}} \rightarrow \operatorname{Ring}_{\Lambda_{R, E}}^{\mathrm{fl}}$, is the right adjoint of the evident forgetful functor.

One then defines $W_{R, E}$ to be the left Kan extension of $W_{R, E}^{\mathrm{fl}}$, now viewed as a functor $\operatorname{Ring}_{R}^{\mathrm{fl}} \rightarrow \operatorname{Ring}_{R}$. This amounts to the following. It is not hard to show that the functor $W_{R, E}^{\mathrm{fl}}$ is representable, that is, there exists an $E$-flat $R$-algebra $\Lambda_{R, E}$ and an isomorphism $W_{R, E}^{\mathrm{fl}}(-)=\operatorname{Hom}\left(\Lambda_{R, E},-\right)$, as set-valued functors. Because $W_{R, E}^{\mathrm{fl}}$ takes values in $R$-algebras, $\Lambda_{R, E}$ carries the structure of a co- $R$-algebra object in Ring $R_{R}^{\mathrm{fl}}$. Because such a structure is described using maps between certain coproducts of $\Lambda_{R, E}$ with itself, and because $\operatorname{Ring}_{R}^{\mathrm{fl}}$ is a full subcategory of Ring ${ }_{R}$ closed under coproducts, $\Lambda_{R, E}$ continues to be a co- $R$-algebra object when viewed as an object of $\operatorname{Ring}_{R}$. Therefore it represents an $R$-algebra-valued functor, and this functor is what $W_{R, E}$ is defined to be.

Since the definition of $W_{R, E}$ in terms of $W_{R, E}^{\mathrm{fl}}$ is of a purely category-theoretic nature, one should view the $E$-flat case as the central one. This is in contrast to the common point of view that the purpose of Witt vector functors is to lift rings from positive characteristic to characteristic zero.

As in the $E$-flat setting, $W_{R, E}$ is the right adjoint of the forgetful functor

$$
\operatorname{Ring}_{\Lambda_{R, E}} \rightarrow \operatorname{Ring}_{R}
$$

but to make sense of this, it is necessary to know the what a $\Lambda_{R, E}$-structure on a general $R$-algebra is. Unfortunately, it is not easy to state the definition, and so we will leave it to the body of the paper. In the $E$-flat setting, it is equivalent to a commuting family of Frobenius lifts indexed by $E$, as above; but in general, it is a slightly stronger structure that is better behaved. When $R$ is $\mathbb{Z}$ and $E$ consists of all maximal ideals of $\mathbb{Z}$, a $\Lambda_{R, E}$-structure is equivalent to a $\lambda$-ring structure in the sense of Grothendieck's Riemann-Roch theory, but this does not admit a simple definition either.

In addition to the right adjoint $W_{R, E}$, the forgetful functor $\operatorname{Ring}_{\Lambda_{R, E}} \rightarrow \operatorname{Ring}_{R}$ has a left adjoint, which we denote by $A \mapsto \Lambda_{R, E} \odot A$. It has a smaller presence in this paper, but it is very important-even in the $p$-typical case, as the work of Buium [1996; 2005] makes clear.

Section 2 defines functors $W_{R, E, n}$, which are truncations of $W_{R, E}$ in the same way that the functors $W_{n}$ above are truncations of $W$. For any $A \in \operatorname{Ring}_{R}^{\mathrm{fl}}$ and $n \in \mathbb{N}^{(E)}$, let $W_{R, E, n}^{\mathrm{fl}}(A)$ denote the image of the subring $W_{R, E}^{\mathrm{fl}}(A) \subseteq A^{\mathbb{N}}$ under the canonical projection

$$
A^{\mathbb{N}^{(E)}} \rightarrow A^{[0, n]},
$$


where

$$
[0, n]=\left\{i \in \mathbb{N}^{(E)} \mid i_{\mathfrak{m}} \leqslant n_{\mathfrak{m}} \text { for all } \mathfrak{m} \in E\right\} .
$$

Then $W_{R, E, n}^{\mathrm{fl}}$ is a functor $\operatorname{Ring}_{R}^{\mathrm{fl}} \rightarrow \operatorname{Ring}_{R}^{\mathrm{fl}}$. It is representable by an $E$-flat $R$-algebra $\Lambda_{R, E, n}$, and we extend it to a functor

$$
W_{R, E, n}: \operatorname{Ring}_{R} \rightarrow \operatorname{Ring}_{R}
$$

by taking its left Kan extension, as above. These truncated functors are related to the original one by the formula

$$
W_{R, E}(A)=\lim _{n} W_{R, E, n}(A) .
$$

Even in the $p$-typical case, this approach to defining the Witt vectors has the advantage over the traditional one that universal properties are emphasized and the particulars of explicit constructions are played down. But this comes at a cost. For instance, it is not obvious that $W_{R, E, n}$ preserves surjectivity of maps. To prove this and other basic facts, it appears necessary to bring back the Witt components $\left(x_{0}, x_{1}, \ldots\right)$ above, at least in some form. To define them, the ideals of $E$ must be principal; the purpose of section 3 is to define them in the minimal case we will need, which is when $E$ consists of a single principal ideal $\mathfrak{m}$. A version of the proof of Witt's theorem then shows there is a unique functorial bijection $A^{\mathbb{N}} \rightarrow W_{R, E}(A)$ such that when $A$ is $E$-flat, the composition $A^{\mathbb{N}} \rightarrow W_{R, E}(A) \subseteq A^{\mathbb{N}}$ satisfies

$$
\left(x_{0}, x_{1}, x_{2}, \ldots\right) \mapsto\left\langle x_{0}, x_{0}^{q}+\pi x_{1}, x_{0}^{q^{2}}+\pi x_{1}^{q}+\pi^{2} x_{2}, \ldots\right\rangle
$$

where $q=[R: \mathfrak{m}]$ and $\pi$ is a fixed generator of $\mathfrak{m}$. We can similarly identify $W_{R, E, n}(A)$ with the quotient $A^{[0, n]}$ consisting of vectors $\left(x_{0}, \ldots, x_{n}\right)$. Let me emphasize that the components $\left(x_{0}, x_{1}, \ldots\right)$ depend on the choice of generator $\pi \in \mathfrak{m}$ in a complex, non-multilinear way. But we can use them to define Verschiebung operators

$$
\begin{aligned}
V_{\mathfrak{m}}^{j}: \mathfrak{m}^{j} \otimes_{R} W_{R, E, n}(A) & \rightarrow W_{R, E, n+j}(A) \\
\pi^{j} \otimes\left(x_{0}, \ldots, x_{n}\right) & \mapsto\left(0, \ldots, 0, x_{0}, \ldots, x_{n}\right),
\end{aligned}
$$

which are independent of the choice of the generator $\pi$. Making that so is the purpose the tensor factor $\mathfrak{m}^{j}$.

When $E$ consists of a single ideal $\mathfrak{m}$ (possibly nonprincipal), section 4 describes $W_{R, E, n}$ in terms of the case where $\mathfrak{m}$ is principal, which is covered by section 3 . This is done by working Zariski locally on $R$. Using the same technique, we will show that the Verschiebung maps as above can be defined when $\mathfrak{m}$ is not assumed to be principal. In fact, there is a unique functorial family of such maps agreeing with the maps defined above. The image of $V_{\mathfrak{m}}^{j}$ is the kernel of the canonical projection $W_{R, E, n+j}(A) \rightarrow W_{R, E, j}(A)$. 
Similarly, section 5 gives a description of $W_{R, E, n}$ when $E$ is general in terms of the case where $E$ consists of a single ideal, which is covered by section 4: if $\mathfrak{m}_{1}, \ldots, \mathfrak{m}_{r}$ are the ideals in the support of $n$, there is a natural isomorphism

$$
W_{R, E, n} \stackrel{\sim}{\longrightarrow} W_{R, \mathfrak{m}_{r}, n_{\mathfrak{m}_{r}}} \circ \cdots \circ W_{R, \mathfrak{m}_{1}, n_{\mathfrak{m}_{1}}} .
$$

Such a description also holds for $W_{R, E}$, though some care must be taken when $E$ is infinite. It is also possible to describe the functor $\Lambda_{R, E} \odot-$, as well as its truncated variants $\Lambda_{R, E, n} \odot-$, in terms of the case where $E$ consists of a single ideal.

Section 6 gives several ring-theoretic facts about $W_{R, E, n}$ which we will need later. For example, this is where we prove that $W_{R, E, n}$ preserves surjectivity. Most of the arguments there appear to require the use of Witt components and the reduction techniques of sections 4 and 5 .

Sections 7-9 prove the main results, which relate Witt vector functors and étale maps. Suppose $E$ consists of a single ideal $\mathfrak{m}$. For any ring $A$ and any integer $n \geqslant 1$, we have a diagram

$$
W_{R, E, n}(A) \stackrel{\alpha_{n}}{\longrightarrow} W_{R, E, n-1}(A) \times A \underset{t \mathrm{opr}_{2}}{\stackrel{s o r_{1}}{\longrightarrow}} A / \mathfrak{m}^{n} A .
$$

When $\mathfrak{m}$ is principal, the maps $\alpha_{n}, s$, and $t$ can be defined in terms of the Witt components relative to a fixed generator $\pi \in \mathfrak{m}$ as follows:

$$
\begin{aligned}
\alpha_{n}:\left(a_{0}, \ldots, a_{n}\right) & \mapsto\left(\left(a_{0}, \ldots, a_{n-1}\right), a_{0}^{q^{n}}+\pi a_{1}^{q^{n-1}} \cdots+\pi^{n} a_{n}\right) \\
s:\left(a_{0}, \ldots, a_{n-1}\right) & \mapsto\left(a_{0}^{q^{n}}+\cdots+\pi^{n-1} a_{n-1}^{q}\right) \bmod \mathfrak{m}^{n} A \\
t: a & \mapsto a \bmod \mathfrak{m}^{n} A .
\end{aligned}
$$

If $A$ is $\mathfrak{m}$-torsion free, (0-0-2) is an equalizer diagram. Figure 1 shows the induced diagram of schemes in the $p$-typical case when $n=1$.

Now let $C$ denote the following category: an object is a pair $(B, \varphi)$, where $B$ is an étale $\left(W_{R, E, n-1}(A) \times A\right)$-algebra and $\varphi$ is an isomorphism of $A / \mathfrak{m}^{n} A$-algebras

$$
A / \mathfrak{m}^{n} A \otimes_{\text {topr }_{2}} B \stackrel{\varphi}{\longrightarrow} A / \mathfrak{m}^{n} A \otimes_{\text {sopr }_{1}} B
$$

and where a morphism $\left(B_{1}, \varphi_{1}\right) \rightarrow\left(B_{2}, \varphi_{2}\right)$ is a $\left(W_{R, E, n-1}(A) \times A\right)$-algebra map $f: B_{1} \rightarrow B_{2}$ such that

$$
\varphi_{2} \circ\left(A / \mathfrak{m}^{n} A \otimes_{\text {topr }_{2}} f\right)=\left(A / \mathfrak{m}^{n} A \otimes_{s \circ \mathrm{pr}_{1}} f\right) \circ \varphi_{1} .
$$

In other words, $C$ is the category of algebras equipped with gluing data relative to the diagram (0-0-2), or equivalently $\mathrm{C}$ is the (weak) fiber product of the category of étale $W_{n-1}(A)$-algebras and the category étale $A$-algebras over the category of étale $A / \mathfrak{m}^{n} A$-algebras via the evident functors. 


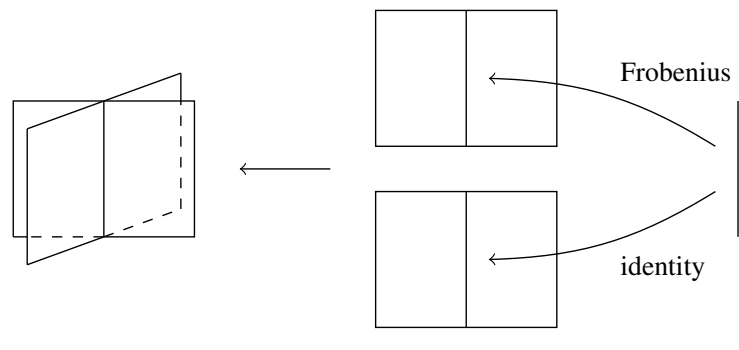

$\operatorname{Spec} W_{1}(A) \longleftarrow \operatorname{Spec}(A \times A) \longleftarrow \operatorname{Spec} A / p A$

Figure 1. As a topological space, $\operatorname{Spec} W_{1}(A)$ (traditionally written $\left.W_{2}(A)\right)$ is two copies of Spec $A$ glued along Spec $A / p A$. This is also true as schemes if we assume that $A$ is $p$-torsion free and we glue transversely and with a Frobenius twist, as indicated. There is a similar description of Spec $W_{n}(A)$ as Spec $W_{n-1}(A)$ glued with $\operatorname{Spec} A$ along Spec $A / p^{n} A$. See the diagram (0-0-2).

Theorem A. The base-change functor from the category of étale $W_{R, E, n}(A)$-algebras to $C$ is an equivalence. If $A$ is $\mathfrak{m}$-torsion free, then a quasi-inverse is given by sending $(B, \varphi)$ to the equalizer of the two maps

$$
B \underset{\varphi \circ\left(1 \otimes \mathrm{id}_{B}\right)}{\stackrel{1 \otimes \mathrm{id}_{B}}{\longrightarrow}} A / \mathfrak{m}^{n} A \otimes_{s \circ \mathrm{pr}_{1}} B .
$$

The first statement can be expressed succinctly in geometric terms; it says that the map $\alpha_{n}$ satisfies effective descent for étale algebras and that descent data is equivalent to gluing data with respect to the diagram (0-0-2). Using theorem $\mathrm{A}$ and induction on $n$, it is in principle possible to reduce questions about étale $W_{n}(A)$ algebras to questions about étale $A$-algebras. This is still true when $E$ consists of more than one ideal, but now by (0-0-1) and induction on $r$.

Section 9 generalizes van der Kallen's theorem [1986, (2.4)] to any $R$ and $E$ :

Theorem B. Let $f: A \rightarrow B$ be an étale morphism of $R$-algebras. Then the map $W_{R, E, n}(f): W_{R, E, n}(A) \rightarrow W_{R, E, n}(B)$ of $R$-algebras is étale.

This result is fundamental in extending Witt constructions beyond affine schemes and will be used often in [Borger 2010]. Van der Kallen's argument, which has an infinitesimal flavor, could be extended to our setting with only minor modifications. ${ }^{1}$ Instead we deduce theorem B from theorem A, so our argument has a globally geometric flavor.

${ }^{1}$ Until recently, [van der Kallen 1986] had escaped the notice of many workers in de Rham-Witt theory, to whom theorem B was unknown even for the $p$-typical Witt vectors. 


\section{Generalized Witt vectors and $\boldsymbol{\Lambda}$-rings}

The purpose of this section is to define our generalized Witt vectors and $\Lambda$-rings. It is largely an expansion in more concrete terms of the portion of [Borger and Wieland 2005] dealing with Witt vectors and $\Lambda$-rings. The approach here will allow us to avoid much of the abstract language of operations on rings, as first introduced in [Tall and Wraith 1970].

For the traditional way of defining $\Lambda$-rings and Witt vectors, see [Bourbaki 1983, XI, §1] and especially the exercises for that section. One can also see Witt's original paper [1937] on $p$-typical Witt vectors and his notes on big Witt vectors [Witt 1998, pp. 157-163].

1.1. Supramaximal ideals. Let us say that an ideal $\mathfrak{m}$ of a ring $R$ is supramaximal if either

(a) $R / \mathfrak{m}$ is a finite field, $R_{\mathfrak{m}}$ is a discrete valuation ring, and $\mathfrak{m}$ is finitely presented as an $R$-module, or

(b) $\mathfrak{m}$ is the unit ideal.

By far the most important example is a maximal ideal with finite residue field in a Dedekind domain. (In fact, all phenomena in this paper occur already over $R=\mathbb{Z}$, and this case covers the classical Witt vectors and $\lambda$-rings.) The reason we allow the unit ideal is only so that a supramaximal ideal remains supramaximal after any localization.

Note that a supramaximal ideal $\mathfrak{m}$ is invertible as an $R$-module. Indeed, locally at $\mathfrak{m}$ it is the maximal ideal of a discrete valuation ring, and away from $\mathfrak{m}$ it is the unit ideal.

1.2. General notation. Fix a ring $R$ and a family $\left(\mathfrak{m}_{\alpha}\right)_{\alpha \in E}$ of pairwise coprime supramaximal ideals of $R$ indexed by a set $E$. Note that because the unit ideal is coprime to itself, it can be repeated any number of times; otherwise the ideals $\mathfrak{m}_{\alpha}$ are distinct. For each $\alpha \in E$, let $q_{\alpha}$ denote the cardinality of $R / \mathfrak{m}_{\alpha}$. We will often abusively speak of $\mathfrak{m}_{\alpha}$ rather than $\alpha$ as being an element of $E$, especially when $\mathfrak{m}_{\alpha}$ is maximal, in which case it comes from a unique $\alpha \in E$.

Let $R[1 / E]$ denote the $R$-algebra whose spectrum is the complement of $E$ in Spec $R$. It is the universal $R$-algebra in which every $\mathfrak{m}_{\alpha}$ becomes the unit ideal. It also has the more concrete description

$$
R[1 / E]=\bigotimes_{\alpha \in E} R\left[1 / \mathfrak{m}_{\alpha}\right],
$$

where the tensor product is over $R$ and $R\left[1 / \mathfrak{m}_{\alpha}\right]$ is defined to be the coequalizer of the maps

$$
\operatorname{Sym}(R) \longrightarrow \operatorname{Sym}\left(\mathfrak{m}_{\alpha}^{-1}\right)
$$


of symmetric algebras, where $\mathfrak{m}_{\alpha}^{-1}$ is the dual of $\mathfrak{m}_{\alpha}$, one of the maps is Sym applied to the canonical map $R \rightarrow \mathfrak{m}_{\alpha}^{-1}$, and the other is the map induced by the $R$-module map $R \rightarrow \operatorname{Sym}\left(\mathfrak{m}_{\alpha}^{-1}\right)$ that sends $1 \in R$ to the element $1 \in \operatorname{Sym}\left(\mathfrak{m}_{\alpha}^{-1}\right)$ in degree zero.

Finally, we write $\mathbb{N}$ for the monoid $\{0,1,2, \ldots\}$ under addition and write $\operatorname{Ring}_{R}$ for the category of $R$-algebras.

1.3. E-flat $R$-modules. Let us say that an $R$-module $M$ is $E$-flat if for all maximal ideals $\mathfrak{m}$ in $E$, the following equivalent conditions are satisfied:

(a) $R_{\mathfrak{m}} \otimes_{R} M$ is a flat $R_{\mathfrak{m}}$-module,

(b) the map $\mathfrak{m} \otimes_{R} M \rightarrow M$ is injective.

The equivalence of these two can be seen as follows. Condition (b) is equivalent to the statement $\operatorname{Tor}_{1}^{R}(R / \mathfrak{m}, M)=0$, which is equivalent to

$$
\operatorname{Tor}_{1}^{R_{\mathfrak{m}}}\left(R / \mathfrak{m}, R_{\mathfrak{m}} \otimes_{R} M\right)=0 .
$$

Since $R_{\mathfrak{m}}$ is a discrete valuation ring, this is equivalent to the $R_{\mathfrak{m}}$-module $R_{m} \otimes_{R} M$ being torsion free and hence flat.

We say an $R$-algebra is $E$-flat if its underlying $R$-module is. Let $\operatorname{Ring}_{R}^{\mathrm{fl}}$ denote the full subcategory of $\operatorname{Ring}_{R}$ consisting of the $E$-flat $R$-algebras.

1.4. Proposition. Any product of E-flat $R$-modules is $E$-flat, and any sub-Rmodule of an E-flat R-module is E-flat.

Proof. We will use condition (b) above. Let $\left(M_{i}\right)_{i \in I}$ be a family of $E$-flat $R$ modules. We want to show that for each maximal ideal $\mathfrak{m}$ in $E$, the composition

$$
\mathfrak{m} \otimes \prod_{i} M_{i} \longrightarrow \prod_{i} \mathfrak{m} \otimes M_{i} \longrightarrow \prod_{i} M_{i}
$$

is injective. Because each $M_{i}$ is $E$-flat, the right-hand map is injective, and so it is enough to show the left-hand map is injective.

Since $\mathfrak{m}$ is assumed to be finitely presented as an $R$-module, we can express it as a cokernel of a map $N^{\prime} \rightarrow N$ of finite free $R$-modules. Then we have the following diagram with exact rows:

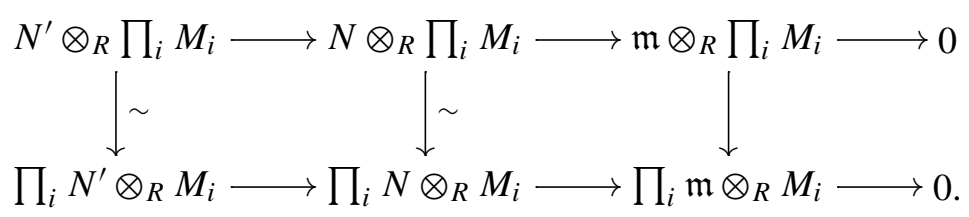

The left two vertical maps are isomorphisms because $N^{\prime}$ and $N$ are finite free. Therefore the rightmost vertical map is an injection (and even an isomorphism). 
Now suppose $M^{\prime}$ is a sub- $R$-module of an $E$-flat $R$-module $M$. Since $\mathfrak{m}$ is an invertible $R$-module, $\mathfrak{m} \otimes_{R} M^{\prime}$ maps injectively to $\mathfrak{m} \otimes_{R} M$. Since $M$ is $E$-flat, $\mathfrak{m} \otimes_{R} M^{\prime}$ further maps injectively to $M$, and hence to $M^{\prime}$.

1.5. $\Psi$-rings. Let $A$ be an $R$-algebra. Let us define a $\Psi_{R, E}$-action, or a $\Psi_{R, E}$-ring structure, on $A$ to be a commuting family of $R$-algebra endomorphisms $\psi_{\alpha}$ indexed by $\alpha \in E$. This is the same as an action of the monoid $\mathbb{N}^{(E)}=\bigoplus_{E} \mathbb{N}$ on $A$. For any element $n \in \mathbb{N}^{(E)}$, we will also write $\psi_{n}$ for the endomorphism of $A$ induced by $n$. A morphism of $\Psi_{R, E}$-rings is defined to be an $\mathbb{N}^{(E)}$-equivariant morphism of rings.

The free $\Psi_{R, E}$-ring on one generator $e$ is $\Psi_{R, E}=R[e]^{\otimes_{R} \mathbb{N}^{(E)}}$, where $\mathbb{N}^{(E)}$ acts on $\Psi_{R, E}$ through its action on itself in the exponent. In particular, $\Psi_{R, E}$ is freely generated as an $R$-algebra by the elements $\psi_{n}(e)$, where $n \in \mathbb{N}^{(E)}$. Then it is natural to write $\psi_{n}=\psi_{n}(e) \in \Psi_{R, E}$ and $\psi_{\alpha}=\psi_{b_{\alpha}} \in \Psi_{R, E}$, where $b_{\alpha} \in \mathbb{N}^{(E)}$ denotes the $\alpha$-th standard basis vector, and $e=\psi_{0} \in \Psi_{R, E}$ for the identity operator.

For any $\Psi_{R, E}$-ring $A$, there is a unique set map

$$
\Psi_{R, E} \times A \stackrel{\circ}{\longrightarrow} A
$$

with the property that for all $\alpha \in E, r \in R, f_{1}, f_{2} \in \Psi_{R, E}, a \in A$ we have

$$
\psi_{\alpha} \circ a=\psi_{\alpha}(a)
$$

and

$$
r \circ a=r, \quad\left(f_{1}+f_{2}\right) \circ a=\left(f_{1} \circ a\right)+\left(f_{2} \circ a\right), \quad\left(f_{1} f_{2}\right) \circ a=\left(f_{1} \circ a\right)\left(f_{2} \circ a\right) .
$$

Taking $A=\Psi_{R, E}$, we get a binary operation $\circ$ on $\Psi_{R, E}$ called composition or plethysm. One can check that this makes $\Psi_{R, E}$ a monoid (noncommutative unless $R=0$ ) with identity $e$ and that (1-5-1) is a monoid action.

In the language of plethystic algebra [Borger and Wieland 2005], we can interpret $\Psi_{R, E}$ as the free $R$-plethory $R\left\langle\psi_{\alpha} \mid \alpha \in E\right\rangle$ on the $R$-algebra endomorphisms $\psi_{\alpha}$. Then a $\Psi_{R, E}$-action in the sense above is the same as a $\Psi_{R, E}$-action in the sense of abstract plethystic algebra. In particular, $\Psi_{R, E}$ can be viewed as the ring of natural unary operations on $\Psi_{R, E}$-rings, and the composition operation $\circ$ above agrees with the usual composition of unary operations. (Compare with 1.18 below.)

1.6. $E$-flat $\Lambda$-rings. Let $A$ be an $R$-algebra which is $E$-flat. Define a $\Lambda_{R, E \text {-action, }}$ or a $\Lambda_{R, E}$-ring structure, on $A$ to be a $\Psi_{R, E}$-action with the following Frobenius lift property: for all $\alpha \in E$, the endomorphism id $\otimes \psi_{\alpha}$ of $R / \mathfrak{m}_{\alpha} \otimes_{R} A$ agrees with the Frobenius map $x \mapsto x^{q_{\alpha}}$. A morphism of $E$-flat $\Lambda_{R, E}$-rings is simply defined to be a morphism of the underlying $\Psi_{R, E}$-rings. Let us denote this category by $\operatorname{Ring}_{\Lambda, E}^{\mathrm{fl}}$. 
1.7. The ghost ring. Since an action of $\Psi_{R, E}$ on an $R$-algebra $A$ is the same as an action (in the category of $R$-algebras) of the monoid $\mathbb{N}^{(E)}$, the forgetful functor from the category of $\Psi_{R, E}$-rings to that of $R$-algebras has a right adjoint given by

$$
A \mapsto \prod_{\mathbb{N}^{(E)}} A=A^{\mathbb{N}^{(E)}},
$$

where $\mathbb{N}^{(E)}$ acts on $A^{\mathbb{N}^{(E)}}$ through its action on itself in the exponent. (This is a general fact about monoid actions in any category with products.) For $a \in A^{\mathbb{N}^{(E)}}$ and $n, n^{\prime} \in \mathbb{N}^{(E)}$, the $n$-th component of $\psi_{n^{\prime}}(a)$ is the $\left(n+n^{\prime}\right)$-th component of $a$.

One might call $A^{\mathbb{N}^{(E)}}$ the cofree $\Psi_{R, E}$-ring on the $R$-algebra $A$. It has traditionally been called the ring of ghost components or ghost vectors. By 1.4, it is $E$-flat if $A$ is.

When $|E|=1$, there is the possibility of confusing the ghost ring $A^{\mathbb{N}}$, which has the product ring structure, with the usual ring $A^{\mathbb{N}}$ of Witt components (see 3.5), which has an exotic ring structure. To prevent this, we will use angle brackets $\left\langle a_{0}, a_{1}, \ldots\right\rangle$ for elements of the ghost ring.

1.8. Witt vectors of $E$-flat rings. Let us now construct the functor $W_{R, E}^{\mathrm{fl}}$. We will show in 1.9 that it is the right adjoint of the forgetful functor from the category of $E$-flat $\Lambda_{R, E}$-rings to that of $E$-flat $R$-algebras. (Further, the flatness will be removed in 1.12.)

Let $A$ be a $E$-flat $R$-algebra. Let $U_{0}(A)$ denote the cofree $\Psi_{R, E}$-ring $A^{\mathbb{N}^{(E)}}$. For any $i \geqslant 0$, let

$$
U_{i+1}(A)=\left\{b \in U_{i}(A) \mid \psi_{\alpha}(b)-b^{q_{\alpha}} \in \mathfrak{m}_{\alpha} U_{i}(A) \text { for all } \alpha \in E\right\} .
$$

This is a sub-R-algebra of $A^{\mathbb{N}^{(E)}}$. Indeed, it is the intersection over $\alpha \in E$ of the equalizers of pairs of $R$-algebra maps

$$
U_{i}(A) \longrightarrow R / \mathfrak{m}_{\alpha} \otimes_{R} U_{i}(A)
$$

given by $x \mapsto 1 \otimes \psi_{\alpha}(x)$ and by $x \mapsto(1 \otimes x)^{q_{\alpha}}$.

Now define

$$
W_{R, E}^{\mathrm{fl}}(A)=\bigcap_{i \geqslant 0} U_{i}(A) .
$$

This is the ring of E-typical Witt vectors with entries in $A$. It is a sub-R-algebra of $A^{\mathbb{N}^{(E)}}$. Observe that $W_{R, E}^{\mathrm{fl}}(A)=A^{\mathbb{N}^{(E)}}$ if $A$ is an $R[1 / E]$-algebra.

1.9. Proposition. (a) $W_{R, E}^{\mathrm{fl}}(A)$ is a sub- $\Psi_{R, E}$-ring of $A^{\mathbb{N}^{(E)}}$.

(b) This $\Psi_{R, E}$-ring structure on $W_{R, E}^{\mathrm{fl}}(A)$ is a $\Lambda_{R, E}$-ring structure.

(c) The induced functor $A \mapsto W_{R, E}^{\mathrm{fl}}(A)$ from $E$-flat $R$-algebras to $E$-flat $\Lambda_{R, E}$ rings is the right adjoint of the forgetful functor. 
Proof. (a) Let us first show by induction that each $U_{i}(A)$ is a sub- $\Psi_{R, E}$-ring of $A^{\mathbb{N}^{(E)}}$. For $i=0$, we have $U_{0}(A)=A^{\mathbb{N}^{(E)}}$, and so it is clear. For $i \geqslant 1$, we use the description of $U_{i+1}(A)$ as the intersection of the equalizers of the pairs of ring maps

$$
U_{i}(A) \longrightarrow R / \mathfrak{m}_{\alpha} \otimes_{R} U_{i}(A)
$$

given in 1.8. Observe that both these ring maps become $\Psi_{R, E}$-ring maps if we give $R / \mathfrak{m}_{\alpha} \otimes_{R} U_{i}(A)$ a $\Psi_{R, E}$-action by defining $\psi_{\beta}: a \otimes x \mapsto a \otimes \psi_{\beta}(x)$, for all $\beta \in E$. Since limits of $\Psi_{R, E}$-rings exist and their underlying rings agree with the limits taken in the category of rings, $U_{i+1}(A)$ is a sub- $\Psi_{R, E}$-ring of $A^{\mathbb{N}^{(E)}}$. Therefore $W_{R, E}^{\mathrm{fl}}(A)$, the intersection of the $U_{i}(A)$, is also a sub- $\Psi_{R, E}$-ring of $A^{\mathbb{N}^{(E)}}$.

(b) It is enough to verify

$$
\psi_{\alpha}(x)-x^{q_{\alpha}} \in \mathfrak{m}_{\alpha} W_{R, E}^{\mathrm{fl}}(A)=\mathfrak{m}_{\alpha} \bigcap_{i \geqslant 0} U_{i}(A),
$$

for all $\alpha \in E$ and $x \in W_{R, E}^{\mathrm{fl}}(A)$. For any $i \geqslant 0$, we know

$$
\psi_{\alpha}(x)-x^{q_{\alpha}} \in \mathfrak{m}_{\alpha} U_{i}(A),
$$

because $x \in W_{R, E}^{\mathrm{fl}}(A) \subseteq U_{i+1}(A)$. Therefore we know

$$
\psi_{\alpha}(x)-x^{q_{\alpha}} \in \bigcap_{i \geqslant 0} \mathfrak{m}_{\alpha} U_{i}(A) .
$$

So, it is enough to show

$$
\mathfrak{m}_{\alpha} \bigcap_{i \geqslant 0} U_{i}(A)=\bigcap_{i \geqslant 0} \mathfrak{m}_{\alpha} U_{i}(A) .
$$

Since $\mathfrak{m}_{\alpha}$ is finitely generated, it is a quotient of a finite free $R$-module $N$. Consider the induced diagram

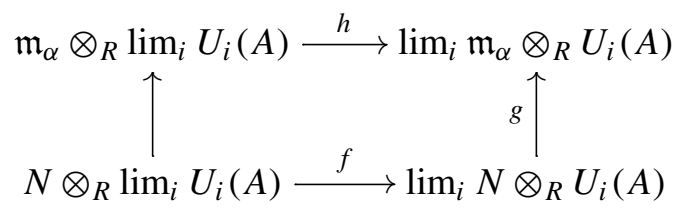

Since $N$ is finite free, $f$ is an isomorphism; since $\mathfrak{m}_{\alpha}$ is projective, the map $N \rightarrow \mathfrak{m}_{\alpha}$ has a section and hence so does $g$. Therefore $g \circ f$ is surjective and hence so is $h$, which implies (1-9-1).

(c) Let $A$ be an $E$-flat $R$-algebra, let $B$ be an $E$-flat $\Lambda_{R, E}$-ring, and let $\bar{\gamma}: B \rightarrow A$ be an $R$-algebra map. By the cofree property of $A^{\mathbb{N}^{(E)}}$, there is a unique $\Psi_{R, E}$-ring map $\gamma: B \rightarrow A^{\mathbb{N}^{(E)}}$ lifting $\bar{\gamma}$. We now only need to show that the image of $\gamma$ is 
contained in $W_{R, E}^{\mathrm{fl}}(A)$. By induction, it is enough to show that if $\operatorname{im}(\gamma) \subseteq U_{i}(A)$, then $\operatorname{im}(\gamma) \subseteq U_{i+1}(A)$.

Let $b$ be an element of $B$. Then for each $\alpha \in E$, we have

$$
\psi_{\alpha}(\gamma(b))-\gamma(b)^{q_{\alpha}}=\gamma\left(\psi_{\alpha}(b)-b^{q_{\alpha}}\right) \in \gamma\left(\mathfrak{m}_{\alpha} B\right) \subseteq \mathfrak{m}_{\alpha} \operatorname{im}(\gamma) \subseteq \mathfrak{m}_{\alpha} U_{i}(A) .
$$

Therefore, by definition of $U_{i+1}(A)$, the element $\gamma(b)$ lies in $U_{i+1}(A)$.

1.10. Exercises. Let $R=\mathbb{Z}$. If $E$ consists of the single ideal $p \mathbb{Z}$, then $W^{\mathrm{fl}}(\mathbb{Z})$ agrees with the subring of the ghost ring $\mathbb{Z}^{\mathbb{N}}$ consisting of vectors $a=\left\langle a_{0}, a_{1}, \ldots\right\rangle$ that satisfy

$$
a_{n} \equiv a_{n+1} \bmod p^{n+1}
$$

for all $n \geqslant 0$. In particular, the elements are $p$-adic Cauchy sequences and the rule $a \mapsto \lim _{n \rightarrow \infty} a_{n}$ defines a surjective ring map $W^{\mathrm{fl}}(\mathbb{Z}) \rightarrow \mathbb{Z}_{p}$.

We can go a step further with $W^{\mathrm{fl}}\left(\mathbb{Z}_{p}\right)$. Let $I$ denote the ideal $p \mathbb{Z}_{p} \times p^{2} \mathbb{Z}_{p} \times \cdots$ in $\mathbb{Z}_{p}^{\mathbb{N}}$. Then $W^{\mathrm{fl}}\left(\mathbb{Z}_{p}\right)$ is isomorphic to the ring $\mathbb{Z}_{p} \oplus I$ with multiplication defined by the formula $(x, y)\left(x^{\prime}, y^{\prime}\right)=\left(x x^{\prime}, x y^{\prime}+y x^{\prime}+y y^{\prime}\right)$.

Now suppose that $E$ consists of all the maximal ideals of $\mathbb{Z}$, and identify $\mathbb{N}^{(E)}$ with the set of positive integers, by unique factorization. Then $W^{\mathrm{fl}}(\mathbb{Z})$ consists of the ghost vectors $\left\langle a_{1}, a_{2}, \ldots\right\rangle$ that satisfy

$$
a_{j} \equiv a_{p j} \bmod p^{1+\operatorname{ord}_{p}(j)}
$$

for all $j \geqslant 1$ and all primes $p$.

1.11. Representing $W^{\mathrm{fl}}$. Let us construct a flat $R$-algebra $\Lambda_{R, E}$ representing the functor $W_{R, E}^{\mathrm{fl}}$. First we will construct objects $\Lambda_{R, E}^{i}$ representing the functors $U_{i}$. For $i=0$, it is clear: $U_{0}$ is represented by $\Lambda_{R, E}^{0}=\Psi_{R, E}$. Now assume $\Lambda_{R, E}^{i}$ has been constructed and that it is a sub- $R$-algebra of $R[1 / E] \otimes_{R} \Psi_{R, E}$ satisfying

$$
R[1 / E] \otimes_{R} \Lambda_{R, E}^{i}=R[1 / E] \otimes_{R} \Psi_{R, E} .
$$

Then let $\Lambda_{R, E}^{i+1}$ denote the sub- $\Lambda_{R, E}^{i}$-algebra of $R[1 / E] \otimes_{R} \Psi_{R, E}$ generated by all elements $\pi^{*} \otimes\left(\psi_{\alpha}(f)-f^{q_{\alpha}}\right)$, where $\pi^{*} \in \mathfrak{m}_{\alpha}^{-1} \subseteq R[1 / E], f \in \Lambda_{R, E}^{i}$, and $\alpha \in E$. Then $\Lambda_{R, E}^{i}$ is flat over $R$. Indeed, it is $E$-flat because it is a sub-R-algebra of $R[1 / E] \otimes_{R} \Psi_{R, E}$, and it is flat away from $E$ because $R[1 / E] \otimes_{R} \Lambda_{R, E}$ agrees with the free $R[1 / E]$-algebra $R[1 / E] \otimes_{R} \Psi_{R, E}$. It also clearly represents $U_{i}$.

Finally, we set

$$
\Lambda_{R, E}=\bigcup_{i \geqslant 0} \Lambda_{R, E}^{i} \subseteq R[1 / E] \otimes_{R} \Psi_{R, E}
$$

It is flat over $R$ because it is a colimit of flat $R$-algebras, and it represents $W_{R, E}^{\mathrm{fl}}$ because each $\Lambda_{R, E}^{i}$ represents $U_{i}$. As an example, if $E=E^{\prime} \sqcup E^{\prime \prime}$, where $E^{\prime \prime}$ 
consists of only copies of the unit ideal, then $\Lambda_{R, E}$ agrees with the monoid algebra $\Lambda_{R, E^{\prime}}\left[\mathbb{N}^{\left(E^{\prime \prime}\right)}\right]$. We will often use the shortened forms $\Lambda_{E}$ or, when $E=\{\mathfrak{m}\}, \Lambda_{\mathfrak{m}}$.

Since $\Lambda_{R, E}$ represents $W^{\mathrm{fl}}$, which takes values in $R$-algebras, $\Lambda_{R, E}$ carries the structure of a co- $R$-algebra object in Ring $R_{R}^{\mathrm{fl}}$. Because $\operatorname{Ring}_{R}^{\mathrm{fl}}$ is closed under coproducts (the tensor product of flat modules being flat), a co-ring structure consists in morphisms

$$
\Delta^{+}, \Delta^{\times}: \Lambda_{R, E} \longrightarrow \Lambda_{R, E} \otimes_{R} \Lambda_{R, E}, \quad \varepsilon^{+}, \varepsilon^{\times}: \Lambda_{R, E} \longrightarrow R
$$

corresponding to addition, multiplication, the additive identity, and the multiplicative identity on the functor $W_{R, E}^{\mathrm{fl}}$. The $R$-linear structure on $W_{R, E}^{\mathrm{fl}}$ corresponds to a morphism

$$
\beta: \Lambda_{R, E} \rightarrow R^{R}=\prod_{R} R .
$$

All these structure maps satisfy the opposite of the $R$-algebra axioms. (In the language of schemes, one would say this makes Spec $\Lambda_{R, E}$ an $R$-algebra scheme over $R$; or in the language of [Borger and Wieland 2005], it makes $\Lambda_{R, E}$ an $R-R$ biring.)

1.12. Definition of $W$ in general. We can view $\Lambda_{R, E}$ as an object of $\operatorname{Ring}_{R}$, instead of Ring $R_{R}^{\mathrm{fl}}$. Then define $W_{R, E}$ as a set-valued functor on $\operatorname{Ring}_{R}$ by

$$
W_{R, E}(A)=\operatorname{Hom}_{\operatorname{Ring}_{R}}\left(\Lambda_{R, E}, A\right) .
$$

The structure maps (1-11-2)-(1-11-3) give $W_{R, E}$ the structure of a functor with values in $R$-algebras:

$$
W_{R, E}: \text { Ring }_{R} \longrightarrow \operatorname{Ring}_{R} .
$$

(Note that here we really use the fact that the coproduct in $\operatorname{Ring}_{R}^{\mathrm{fl}}$ agrees with that in $\operatorname{Ring}_{R}$. In 1.11, it was used only to justify the symbol $\otimes$ for the coproduct.)

For any $A \in \operatorname{Ring}_{R}$, let us call the $W_{R, E}(A)$ the $R$-algebra of E-typical Witt vectors with entries in $A$. Its restriction to Ring $R_{R}^{\mathrm{fl}}$ agrees with $W_{R, E}^{\mathrm{fl}}$ because Ring ${ }_{R}^{\mathrm{fl}}$ is a full subcategory of Ring ${ }_{R}$.

We will often write $W_{E}$ or $W$ for $W_{R, E}$ when there is no risk of confusion. When $E$ consists of a single ideal $\mathfrak{m}$, we will also write $W_{R, \mathfrak{m}}$ or $W_{\mathfrak{m}}$.

1.13. Remark: Kan extensions. In categorical terms, $W_{R, E}$ is the left Kan extension of $i \circ W_{R, E}^{\mathrm{fl}}$ along the inclusion functor $i$ :

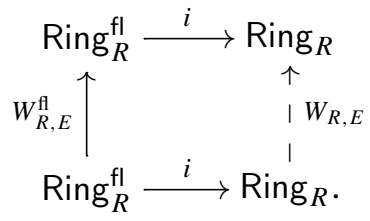


(See [Borceux 1994a, 3.7], for example, for the general theory of Kan extensions.) I mention this only to emphasize that the passage from the $E$-flat case to the general case is by a purely category-theoretic process, and hence the heart of the theory lies in the $E$-flat case. This is in contrast to the common point of view that the purpose of Witt vector functors is to lift rings from positive characteristic to characteristic zero.

1.14. Ghost map $w$. The ghost map

$$
w: W_{R, E}(A) \longrightarrow \prod_{\mathbb{N}(E)} A
$$

is the natural map induced by the universal property of Kan extensions applied to the inclusion maps $W_{R, E}^{\mathrm{fl}}(A) \rightarrow \prod_{\mathbb{N}^{(E)}} A$, which are functorial in $A$. Equivalently, it is the morphism of functors induced by the map

$$
\Psi_{R, E}=\Lambda_{R, E}^{0} \longrightarrow \Lambda_{R, E}
$$

of representing objects. When $A$ is $E$-flat, it is harmless to identify $w$ with the inclusion map.

1.15. Example: $p$-typical and big Witt vectors. Suppose $R$ is $\mathbb{Z}$. If $E$ consists of the single ideal $p \mathbb{Z}$, then $W$ agrees with the classical $p$-typical Witt vector functor [Witt 1937]. Indeed, for $p$-torsion free rings $A$, this follows from Cartier's lemma, which says that the traditionally defined $p$-typical Witt vector functor restricted to the category of $p$-torsion-free rings has the same universal property as $W^{\mathrm{fl}}$. (See [Bourbaki 1983, IX.44, exercice 14] or [Lazard 1975, VII§4].) Therefore, they are isomorphic functors. For $A$ general, one just observes that the traditional functor is represented by the ring $\mathbb{Z}\left[x_{0}, x_{1}, \ldots\right]$, which is $p$-torsion free, and so it is the left Kan extension of its restriction to the category of $p$-torsion-free rings. Therefore it agrees with $W$ as defined here.

Another proof of this is given in 3.5. It makes a direct connection with the traditional Witt components, rather than going through the universal property.

Suppose instead that $E$ is the family of all maximal ideals of $\mathbb{Z}$. Then $W$ agrees with the classical big Witt vector functor. As above, this can be shown by reducing to the torsion-free case and then citing the analogue of Cartier's lemma. (Which version of Cartier's lemma depends on how we define the classical big Witt vector functor. If we use generalized Witt polynomials, we need [Bourbaki 1983, IX.55, exercise 41b]. If it is defined as the cofree $\lambda$-ring functor, as in [Grothendieck 1958], then we need Wilkerson's theorem [1982, Proposition 1.2].)

Finally, we will see in 3.5 that when $R$ is a complete discrete valuation ring and $E$ consists of the maximal ideal of $R$, then $W$ agrees with Hazewinkel's ramified Witt vector functor [Hazewinkel 1978, (18.6.13)]. 
1.16. Comonad structure on $W$. The functor $W^{\mathrm{fl}}: \operatorname{Ring}_{R}^{\mathrm{fl}} \rightarrow \operatorname{Ring}_{R}^{\mathrm{fl}}$ is naturally a comonad, being the composition of a functor (the forgetful one) with its right adjoint, and this comonad structure prolongs naturally to $W_{R, E}$. The reason for this can be expressed in two ways-in terms of Kan extensions or in terms of representing objects.

The first way is to invoke the general fact that $W_{R, E}$, as the Kan extension of the comonad $W_{R, E}^{\mathrm{fl}}$, has a natural comonad structure. This uses the commutativity of (1-13-1) and the fullness and faithfulness of $i$. The other way is to translate the structure on $W^{\mathrm{fl}}$ of being a comonad into a structure on its representing object $\Lambda_{R, E}$. One then observes that this is exactly the structure for the underlying $R$ algebra $i\left(\Lambda_{R, E}\right)$ to represent a comonad on $\operatorname{Ring}_{R}$. (This is called an $R$-plethory structure in [Borger and Wieland 2005].)

1.17. $\Lambda$-rings. The category $\operatorname{Ring}_{\Lambda_{R, E}}$ of $\Lambda_{R, E}$-rings is by definition the category of coalgebras for the comonad $W_{R, E}$, that is, the category of $R$-algebras equipped with a coaction of the comonad $W_{R, E}$. Since $W_{R, E}$ extends $W_{R, E}^{\mathrm{fl}}$, a $\Lambda_{R, E}$-ring structure on an $E$-flat $R$-algebra $A$ is the same as a commuting family of Frobenius lifts $\psi_{\alpha}$.

When $R=\mathbb{Z}$ and $E$ is the family of all maximal ideals of $\mathbb{Z}$, then a $\Lambda$-ring is the same as a $\lambda$-ring in the sense of [Grothendieck 1958] (and originally called a "special $\lambda$-ring"). In the $E$-flat case, this is Wilkerson's theorem [1982, Proposition 1.2]. The proof is an exercise in symmetric functions, but the deeper meaning eludes me. The general case follows from the $E$-flat case by category theory, as in 1.15 .

1.18. Free $\Lambda$-rings and $\Lambda \odot-$. Since $W_{R, E}$ is a representable comonad on $\operatorname{Ring}_{R}$, the forgetful functor from the category of $\Lambda_{R, E}$-rings to the category of $R$-algebras has a left adjoint denoted $\Lambda_{R, E} \odot-$. This follows either from the adjoint functor theorem in category theory [Borceux 1994a, 3.3.3], or by simply writing down the adjoint in terms of generators and relations, as in [Borger and Wieland 2005, 1.3]. The second approach involves the $R$-plethory structure on $\Lambda_{R, E}$, and is similar to the description of tensor products, free differential rings, and so on in terms of generators and relations.

The functor $\Lambda_{R, E} \odot-$, viewed as an endofunctor on the category of $R$-algebras, is naturally a monad, simply because it is the left adjoint of the comonad $W_{R, E}$. Further, the category of algebras for this monad is naturally equivalent to $\operatorname{Ring}_{\Lambda_{R, E}}$. This can be proved using Beck's theorem [Borceux 1994b, 4.4.4], and is the same as the fact that the category of $K$-modules, for any ring $K$, can be defined as the category of algebras for the monad $K \otimes-$ or coalgebras for the comonad $\operatorname{Hom}(K,-)$. 
We can interpret elements of $\Lambda_{R, E}$ as natural operations on $\Lambda_{R, E}$-rings. Indeed, a $\Lambda_{R, E}$-ring structure on a ring $A$ is by definition a (type of) map $A \rightarrow W_{R, E}(A)$. It therefore induces a set map

$$
\Lambda_{R, E} \times A \longrightarrow \Lambda_{R, E} \times W_{R, E}(A)=\Lambda_{R, E} \times \operatorname{Hom}_{R}\left(\Lambda_{R, E}, A\right) \longrightarrow A,
$$

which is functorial in $A$. In particular, if we take $A=\Lambda_{R, E}$, we get a set map

$$
\Lambda_{R, E} \times \Lambda_{R, E} \stackrel{\circ}{\longrightarrow} \Lambda_{R, E} .
$$

It agrees with the restriction of the composition map $\circ$ on $\Psi_{R[1 / E], E}=R[1 / E] \otimes_{R}$ $\Psi_{R, E}$ given in 1.5. In particular, it is associative with identity $e$.

In fact, all natural operations on $\Lambda_{R, E}$-rings come from $\Lambda_{R, E}$ in this way. See [Borger and Wieland 2005] for an abstract account from this point of view.

1.19. Remark: identity-based approaches. It is possible to set up the theory of $\Lambda_{R, E}$-rings more concretely using universal identities rather than category theory. (See [Buium 1996; Buium and Simanca 2009; Joyal 1985a; 1985b], for example.) In this subsection, I will say something about that point of view and its relation to the category-theoretic one, but it will not be used elsewhere in this paper.

First suppose that for each $\alpha \in E$, the ideal $\mathfrak{m}_{\alpha}$ is generated by a single element $\pi_{\alpha}$. For any $\Lambda_{R, E}$-ring $A$ and any element $a \in A$, there exists an element $\delta_{\alpha}(a) \in A$ such that

$$
\psi_{\alpha}(a)=a^{q_{\alpha}}+\pi_{\alpha} \delta_{\alpha}(a) .
$$

If we now assume that $A$ is $E$-flat, the element $\delta_{\alpha}(a)$ is uniquely determined by this equation, and therefore $\delta_{\alpha}$ defines an operator on $A$ :

$$
\delta_{\alpha}(a)=\frac{\psi_{\alpha}(a)-a^{q_{\alpha}}}{\pi_{\alpha}} .
$$

Observe that if the integer $q_{\alpha}$ maps to 0 in $R$, for example when $R$ is a ring of integers in a function field, then $\delta_{\alpha}$ is additive; but otherwise it essentially never is. (Also note that $\delta_{\alpha}$ is the same as the operator $\theta_{\pi_{\alpha}, 1}$ defined in 3.1 below.)

Conversely, if $A$ is an $E$-flat $R$-algebra, equipped with operators $\delta_{\alpha}$, there is at most one $\Lambda_{R, E}$-ring structure on $A$ whose $\delta_{\alpha}$-operators are the given ones. To say when such a $\Lambda_{R, E}$-ring structure exists, we only need to express in terms of the operators $\delta_{\alpha}$ the condition that the operators $\psi_{\alpha}$ be commuting $R$-algebra homomorphisms. After dividing by any accumulated factors of $\pi_{\alpha}$, this gives the identities of Buium-Simanca [2009, Definition 2.1]:

$$
\begin{aligned}
\delta_{\alpha}(r) & =\frac{r-r^{q_{\alpha}}}{\pi_{\alpha}}, \quad \text { for } r \in R, \\
\delta_{\alpha}(a+b) & =\delta_{\alpha}(a)+\delta_{\alpha}(b)+C_{\alpha}(a, b), \\
\delta_{\alpha}(a b) & =\delta_{\alpha}(a) b^{q_{\alpha}}+a^{q_{\alpha}} \delta_{\alpha}(b)+\pi_{\alpha} \delta_{\alpha}(a) \delta_{\alpha}(b),
\end{aligned}
$$




$$
\delta_{\alpha} \circ \delta_{\alpha^{\prime}}(a)=\delta_{\alpha^{\prime}} \circ \delta_{\alpha}(a)+C_{\alpha, \alpha^{\prime}}\left(a, \delta_{\alpha}(a), \delta_{\alpha^{\prime}}(a)\right),
$$

where

$$
C_{\alpha}(x, y)=\frac{x^{q_{\alpha}}+y^{q_{\alpha}}-(x+y)^{q_{\alpha}}}{\pi_{\alpha}}=-\sum_{i=1}^{q_{\alpha}-1} \frac{1}{\pi_{\alpha}}\left(\begin{array}{c}
q_{\alpha} \\
i
\end{array}\right) x^{q_{\alpha}-i} y^{i}
$$

and

$C_{\alpha, \alpha^{\prime}}(x, y, z)$

$$
=\frac{C_{\alpha^{\prime}}\left(x^{q_{\alpha}}, \pi_{\alpha} y\right)}{\pi_{\alpha}}-\frac{C_{\alpha}\left(x^{q_{\alpha^{\prime}}}, \pi_{\alpha^{\prime}} z\right)}{\pi_{\alpha^{\prime}}}-\frac{\delta_{\alpha}\left(\pi_{\alpha^{\prime}}\right)}{\pi_{\alpha^{\prime}}} z^{q_{\alpha}}+\frac{\delta_{\alpha^{\prime}}\left(\pi_{\alpha}\right)}{\pi_{\alpha}} y^{q_{\alpha^{\prime}}} .
$$

One can easily check that the coefficients of these polynomials are elements of $R$.

For any $R$-algebra $A$, let us define a $\delta_{R, E}$-structure on $A$ to be a family of operators $\delta_{\alpha}$ satisfying the axioms above. Thus, if $A$ is an $E$-flat $R$-algebra, then a $\Lambda_{R, E}$-structure-by definition a commuting family of Frobenius lifts indexed by $E$-is equivalent to a $\delta_{R, E}$-structure. The point of all this, then, is that if we no longer require $A$ to be $E$-flat, a $\delta_{R, E}$-structure is generally stronger than having a commuting family of Frobenius lifts, but it is still equivalent to having a $\Lambda_{R, E}$-structure. This offers another point of view on the difference between a $\Lambda_{R, E}$-structure and a commuting family of Frobenius lifts: A $\delta_{R, E}$-structure is well behaved from the point of view of universal algebra (and hence so is a $\Lambda_{R, E^{-}}$ structure) because it is given by operators $\delta_{\alpha}$ whose effect on the ring structure is described by universal identities, as above; but the structure of a commuting family of Frobenius lifts does not have this property because of the existential quantifier hidden in the word lift.

The equivalence between $\delta_{R, E}$-structures and $\Lambda_{R, E}$-structures can be seen as follows. For $E$-flat $R$-algebras $A$, it was explained above. For general $A$, the equivalence can be shown by checking that the cofree $\delta_{R, E}$-ring functor is represented by an $E$-flat $R$-algebra (in fact, a free one). It therefore agrees with the left Kan extension of its restriction to the category of $E$-flat algebras, and hence agrees with $W_{R, E}$.

We could extend the identity-based approach to the case where the ideals $\mathfrak{m}_{\alpha}$ are not principal, but then we would need operators

$$
\delta_{\alpha, \pi_{\alpha}^{*}}(x)=\pi_{\alpha}^{*}\left(\psi_{\alpha}(x)-x^{q_{\alpha}}\right)
$$

for every element $\pi_{\alpha}^{*} \in \mathfrak{m}_{\alpha}^{-1}$, or at least for those in a chosen generating set of $\mathfrak{m}_{\alpha}^{-1}$, and we would need additional axioms relating them. A particularly convenient generating set of $\mathfrak{m}_{\alpha}^{-1}$ is one of the form $\left\{1, \pi_{\alpha}^{*}\right\}$, which always exists. Further, for each $\alpha \in E$, it is enough to use the operators $\psi_{\alpha}$ and $\delta_{\alpha, \pi_{\alpha}^{*}}$ instead of $\delta_{\alpha, 1}$ and $\delta_{\alpha, \pi_{\alpha}^{*}}$, 
because $\delta_{\alpha, 1}$ can be expressed in terms of $\psi_{\alpha}$, by (1-19-7). Therefore if we fix elements $\pi_{\alpha}^{*} \in \mathfrak{m}_{\alpha}^{-1}$ which are $R$-module generators modulo 1 , the relations needed for the generating set $\bigcup_{\alpha \in E}\left\{\psi_{\alpha}, \delta_{\alpha, \pi_{\alpha}^{*}}\right\}$ of operators are those in (1-19-1)-(1-19-6) but one needs to make the following changes: for each $\alpha \in E$, replace each occurrence of $\pi_{\alpha}^{-1}$ with $\pi_{\alpha}^{*}$, and add axioms that $\psi_{\alpha}$ is an $R$-algebra homomorphism, that $\psi_{\alpha}$ commutes with all $\psi_{\alpha^{\prime}}$ and all $\delta_{\alpha^{\prime}, \pi_{\alpha^{\prime}}^{*}}$, and that (1-19-7) holds.

When $R$ is an $\mathbb{F}_{p}$-algebra for some prime number $p$, the polynomials $C_{\alpha}(x, y)$ are zero and the axioms above simplify considerably. In particular, the operators $\delta_{\alpha}$ are additive, and so it is possible to describe a $\Lambda_{R, E}$-structure using a cocommutative twisted bialgebra, the additive bialgebra of the plethory $\Lambda_{R, E}$. See [Borger and Wieland 2005, sections 2 and 10].

1.20. Localization of the ring $R$ of scalars. Let $R^{\prime}$ be an $E$-flat $R$-algebra such that the structure map $R \rightarrow R^{\prime}$ is an epimorphism of rings. (For example, the map Spec $R^{\prime} \rightarrow$ Spec $R$ could be an open immersion.) Then the family $\left(\mathfrak{m}_{\alpha}\right)_{\alpha \in E}$ induces a family $\left(\mathfrak{m}_{\alpha}^{\prime}\right)_{\alpha \in E}$ of ideals of $R^{\prime}$, where $\mathfrak{m}_{\alpha}^{\prime}=\mathfrak{m}_{\alpha} R^{\prime}$. By the assumptions on $R^{\prime}$, each $\mathfrak{m}_{\alpha}^{\prime}$ is supramaximal. Let us write $E^{\prime}=E$ and use the notation $E^{\prime}$ for the index set of the $\mathfrak{m}_{\alpha}^{\prime}$.

Let us construct an isomorphism:

$$
R^{\prime} \otimes_{R} \Lambda_{R, E} \stackrel{\sim}{\longrightarrow} \Lambda_{R^{\prime}, E^{\prime}}
$$

The category $\operatorname{Ring}_{\Lambda_{R^{\prime}, E^{\prime}}}^{\mathrm{fl}}$ (see 1.6) is a subcategory of the category of $\operatorname{Ring}_{\Lambda_{R, E}}^{\mathrm{fl}}$. Indeed, any object $A^{\prime} \in \operatorname{Ring}_{\Lambda_{R^{\prime}, E^{\prime}}}^{\mathrm{fl}}$ is an $R$-algebra with endomorphisms $\psi_{\mathfrak{m}_{\alpha}}$, for each $\alpha \in E$. These endomorphisms are again commuting Frobenius lifts, simply because $A^{\prime} / \mathfrak{m}_{\alpha}^{\prime} A^{\prime}=A^{\prime} / \mathfrak{m}_{\alpha} A^{\prime}$. Since $A^{\prime}$ is $E^{\prime}$-flat (and by the assumptions on $R^{\prime}$ ), $A^{\prime}$ is $E$-flat. Therefore, it can be viewed as a $\Lambda_{R, E}$-ring.

Further, Ring $\Lambda_{R^{\prime}, E^{\prime}}^{\mathrm{fl}}$ agrees with the subcategory of Ring $\Lambda_{R, E}^{\mathrm{fl}}$ consisting of objects $A$ whose structure map $R \rightarrow A$ factors through $R^{\prime}$, necessarily uniquely. Now consider the underlying-set functor on this category. From the definition of Ring $\Lambda_{R^{\prime}, E^{\prime}}^{\mathrm{fl}}$, this functor is represented by the right-hand side of (1-20-1), and from the second description, it is represented by the left-hand side. Let (1-20-1) be the induced isomorphism on representing objects. It sends an element $r^{\prime} \otimes f$ to $r^{\prime} f$.

The isomorphism of represented functors which is induced by (1-20-1) gives natural maps

$$
W_{R^{\prime}, E^{\prime}}\left(A^{\prime}\right) \stackrel{\sim}{\longrightarrow} W_{R, E}\left(A^{\prime}\right)
$$

for $R^{\prime}$-algebras $A^{\prime}$.

Finally, let us show that for any $R^{\prime}$-algebra $B^{\prime}$, the canonical map

$$
\Lambda_{R, E} \odot B^{\prime} \stackrel{\sim}{\longrightarrow} \Lambda_{R^{\prime}, E^{\prime}} \odot B^{\prime}
$$


is an isomorphism. It is enough to show that for any $R^{\prime}$-algebra $A^{\prime}$, the induced map

$$
\operatorname{Hom}_{R^{\prime}}\left(\Lambda_{R^{\prime}, E^{\prime}} \odot B^{\prime}, A^{\prime}\right) \longrightarrow \operatorname{Hom}_{R^{\prime}}\left(\Lambda_{R, E} \odot B^{\prime}, A^{\prime}\right)
$$

is a bijection. Since Ring $R_{R^{\prime}}$ is a full subcategory of Ring ${ }_{R}$, the right-hand side agrees with $\operatorname{Hom}_{R}\left(\Lambda_{R, E} \odot B^{\prime}, A^{\prime}\right)$, and so the map above is an isomorphism by $(1-20-2)$.

1.21. Teichmüller lifts. Let $A$ be an $R$-algebra, let $A^{\circ}$ denote the commutative monoid of all elements of $A$ under multiplication, and let $R\left[A^{\circ}\right]$ denote the monoid algebra on $A^{\circ}$. Then for each $\alpha \in E$, the monoid endomorphism $a \mapsto a^{q_{\alpha}}$ of $A^{\circ}$ induces an $R$-algebra endomorphism $\psi_{\alpha}$ of $R\left[A^{\circ}\right]$ which reduces to the $q_{\alpha^{-}}$ th power map modulo $\mathfrak{m}_{\alpha}$. Since $R\left[A^{\circ}\right]$ is free as an $R$-module, it is flat. And since the various $\psi_{\alpha}$ commute with each other, they provide $R\left[A^{\circ}\right]$ with a $\Lambda_{R, E^{-}}$ structure. Combined with the $R$-algebra map $R\left[A^{\circ}\right] \rightarrow A$ given by the counit of the evident adjunction, this gives, by the right-adjoint property of $W_{R, E}$, a $\Lambda_{R, E}$-ring map $t: R\left[A^{\circ}\right] \rightarrow W_{R, E}(A)$. We write the composite monoid map

$$
A^{\circ} \stackrel{\text { unit }}{\longrightarrow} R\left[A^{\circ}\right]^{\circ} \stackrel{t^{\circ}}{\longrightarrow} W_{R, E}(A)^{\circ}
$$

as simply $a \mapsto[a]$. It is a section of the $R$-algebra map $w_{0}: W_{R, E}(A) \rightarrow A$ and is easily seen to be functorial in $A$. The element $[a]$ is called the Teichmüller lift of $a$.

\section{Grading and truncations}

2.1. Ordering on $\mathbb{Z}^{(E)}$. For two elements $n^{\prime}, n \in \mathbb{Z}^{(E)}=\bigoplus_{E} \mathbb{Z}$, write $n^{\prime} \leqslant n$ if we have $n_{\alpha}^{\prime} \leqslant n_{\alpha}$ for all $\alpha \in E$. Also put

$$
[0, n]=\left\{n^{\prime} \in \mathbb{N}^{(E)} \mid n^{\prime} \leqslant n\right\} .
$$

2.2. Truncations. We have the following decomposition of $\Psi_{R, E}$ :

$$
\Psi_{R, E}=\bigotimes_{\alpha \in E} \bigotimes_{i \in \mathbb{N}} R\left[\psi_{\alpha}^{\circ i}\right]=\bigotimes_{n \in \mathbb{N}^{(E)}} R\left[\psi_{n}\right]=R\left[\psi_{n} \mid n \in \mathbb{N}^{(E)}\right]
$$

(Thus, $\Psi_{R, E}$ is an $\mathbb{N}^{(E)}$-indexed coproduct in the category of $R$-algebras, much like graded rings are monoid-indexed coproducts in the category of modules. One might say that $\Psi_{R, E}$ is an $\mathbb{N}^{(E)}$-graded plethory. This point of view will not be used below.) For each $n \in \mathbb{Z}^{(E)}$, put

$$
\Psi_{R, E, n}=\bigotimes_{\alpha \in E} \bigotimes_{0 \leqslant i \leqslant n_{\alpha}} R\left[\psi_{\alpha}^{\circ i}\right]=\bigotimes_{n^{\prime} \in[0, n]} R\left[\psi_{n^{\prime}}\right]=R\left[\psi_{n^{\prime}} \mid n^{\prime} \in[0, n]\right] .
$$

Then $\Psi_{R, E, n}$ represents the Ring $R_{R}$-valued functor that sends $A$ to the product ring $A^{[0, n]}$, which is naturally a quotient of $A^{\mathbb{N}^{(E)}}$. 
Define a similar filtration on $\Lambda_{R, E}$ by

$$
\Lambda_{R, E, n}=\Lambda_{R, E} \cap\left(R[1 / E] \otimes_{R} \Psi_{R, E, n}\right) .
$$

We will often use the shortened forms $\Lambda_{E, n}, \Psi_{E, n}, \Lambda_{\mathfrak{m}, n}, \Psi_{\mathfrak{m}, n}$, and so on.

2.3. Proposition. (a) For each $n \in \mathbb{N}^{(E)}$, the $R$-scheme Spec $\Lambda_{R, E, n}$ admits a unique structure of an $R$-algebra object in the category of $R$-schemes such that the map Spec $\Lambda_{R, E} \rightarrow \operatorname{Spec} \Lambda_{R, E, n}$ induced by the inclusion $\Lambda_{R, E, n} \subseteq$ $\Lambda_{R, E}$ is a homomorphism of $R$-algebra schemes over $R$.

(b) For each $m, n \in \mathbb{N}^{(E)}$, we have

$$
\Lambda_{R, E, m} \circ \Lambda_{R, E, n} \subseteq \Lambda_{R, E, m+n},
$$

where o denotes the composition map of (1-18-1).

Proof. (a) Write $\Lambda=\Lambda_{R, E}, \Lambda_{n}=\Lambda_{R, E, n}$, and so on. First observe that, for any integer $i \geqslant 0$, all the maps in the diagram

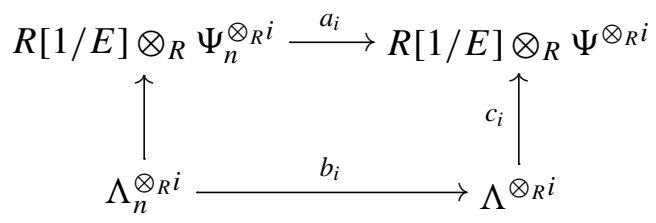

are injective. Indeed, $a_{i}$ clearly is; the vertical maps are because they become isomorphisms after base change to $R[1 / E]$ and because $\Lambda_{n}$ and $\Lambda$ are $E$-flat; and it follows formally that $b_{i}$ is injective. Then the uniqueness of the desired $R$-algebra scheme structure on Spec $\Lambda_{n}$, follows from the injectivity of $b_{2}$.

Now consider existence. Let

$$
\Delta: R[1 / E] \otimes_{R} \Psi \longrightarrow R[1 / E] \otimes_{R} \Psi \otimes_{R} \Psi
$$

denote the ring map that induces the addition (resp. multiplication) map on the ring scheme Spec $R[1 / E] \otimes_{R} \Psi_{R}$. To show that the desired addition and multiplication maps on $\operatorname{Spec} \Lambda_{n}$ exist, it is enough to show

$$
\Delta\left(\Lambda_{n}\right) \subseteq \Lambda_{n} \otimes_{R} \Lambda_{n} .
$$

In fact, once we do this, we will be done: because each $c_{i} \circ b_{i}$ is injective, the ring axioms (associativity, distributivity, and so on) will follow from those on Spec $R[1 / E] \otimes_{R} \Psi$.

The map $\Delta$ sends $\psi_{\alpha}$ to $\psi_{\alpha} \otimes 1+1 \otimes \psi_{\alpha}$ (resp. $\psi_{\alpha} \otimes \psi_{\alpha}$ ). Therefore we have

$$
\Delta\left(R[1 / E] \otimes_{R} \Psi_{n}\right) \subseteq R[1 / E] \otimes_{R} \Psi_{n} \otimes_{R} \Psi_{n},
$$


and hence

$$
\Delta\left(\Lambda_{n}\right) \subseteq \Lambda^{\otimes_{R} 2} \cap\left(R[1 / E] \otimes_{R} \Psi_{n}^{\otimes_{R} 2}\right)=\Lambda_{n}^{\otimes_{R} 2} .
$$

This establishes (2-3-2) and hence completes the proof of (a).

(b) Combine the definition (2-2-1) with the inclusion

$$
\left(R[1 / E] \otimes_{R} \Psi_{m}\right) \circ\left(R[1 / E] \otimes_{R} \Psi_{n}\right) \subseteq\left(R[1 / E] \otimes_{R} \Psi_{m+n}\right)
$$

and the inclusion $\Lambda_{m} \circ \Lambda_{n} \subseteq \Lambda$.

2.4. Witt vectors of finite length. Let $W_{R, E, n}$ denote the functor $\operatorname{Ring}_{R} \rightarrow \operatorname{Ring}_{R}$ represented by $\Lambda_{R, E, n}$ :

$$
W_{R, E, n}(A)=\operatorname{Hom}_{R}\left(\Lambda_{R, E, n}, A\right) .
$$

We call $W_{R, E, n}$ the E-typical Witt vector functor of length $n$. As in 1.12, we will often write $W_{E, n}$ or $W_{n}$; when $E=\{\mathfrak{m}\}$, we will also write $W_{R, \mathfrak{m}, n}$ or $W_{\mathfrak{m}, n}$. We then have

$$
W_{R, E}(A)=\lim _{n} W_{R, E, n}(A) .
$$

(Note that it is often better to view $W_{R, E}(A)$ as a pro-ring than to actually take the limit. If we preferred topological rings to pro-rings, we could take the limit and endow it with the natural pro-discrete topology.) It follows from 4.4 and (5-4-2) below that the maps in this projective system are surjective.

The (truncated) ghost map

$$
w_{\leqslant n}: W_{R, E, n}(A) \longrightarrow A^{[0, n]},
$$

is the one induced by the inclusion $\Psi_{R, E, n} \subseteq \Lambda_{R, E, n}$ of representing objects. For any $i \in[0, n]$, the composition $w_{\leqslant n}$ with the projection onto the $i$-th factor gives another natural map

$$
w_{i}: W_{R, E, n}(A) \longrightarrow A .
$$

Also the containment (2-3-1) induces an $R$-algebra map

$$
W_{R, E, m+n}(A) \longrightarrow W_{R, E, n}\left(W_{R, E, m}(A)\right)
$$

which sends an element $a: \Lambda_{R, E, m+n} \rightarrow A$ of $W_{R, E, m+n}(A)$ to the map $\gamma \mapsto$ $[\beta \mapsto a(\beta \circ \gamma)]$, for variables $\gamma \in \Lambda_{R, E, n}$ and $\beta \in \Lambda_{R, E, m}$. We will call (2-4-5) co-plethysm. It agrees with the map of functors induced by the map

$$
\Lambda_{R, E, m} \odot \Lambda_{R, E, n} \longrightarrow \Lambda_{R, E, m+n}, \quad \beta \odot \gamma \mapsto \beta \circ \gamma
$$

on representing objects, where $\beta \odot \gamma$ is defined as in [Borger and Wieland 2005].

Finally, observe that for any element $f \in \Lambda_{R, E, n}$ the natural $\Lambda_{R, E}$-ring operation

$$
f: W_{R, E}(A) \rightarrow W_{R, E}(A)
$$


(a map of sets) descends to a map

$$
f: W_{R, E, m+n}(A) \rightarrow W_{R, E, m}(A) .
$$

Indeed, it is the composition

$$
\begin{array}{r}
W_{R, E, m+n}(A) \stackrel{(2-4-5)}{\longrightarrow} W_{R, E, n}\left(W_{R, E, m}(A)\right)=\operatorname{Hom}\left(\Lambda_{R, E, n}, W_{R, E, m}(A)\right) \\
\stackrel{-(f)}{\longrightarrow} W_{R, E, m}(A),
\end{array}
$$

where $-(f)$ denotes the map that evaluates at $f$. Particularly important is the example $f=\psi_{n}$, where the induced map

$$
\psi_{n}: W_{R, E, m+n}(A) \rightarrow W_{R, E, m}(A)
$$

is a ring homomorphism.

2.5. Remark: traditional versus normalized indexing. Consider the $p$-typical Witt vectors, where $R$ is $\mathbb{Z}$ and $E$ consists of the single ideal $p \mathbb{Z}$. Let $W_{n}^{\prime}$ denote Witt's functor, as defined in [Witt 1937]. So, for example,

$$
W_{n}^{\prime}\left(\mathbb{F}_{p}\right)=\mathbb{Z} / p^{n} \mathbb{Z} \text {. }
$$

In 3.5, we will construct an isomorphism $W_{n+1}^{\prime} \cong W_{n}$. Thus, up to a normalization of indices, our truncated Witt functors agree with Witt's.

The reason for this normalization is to make the indexing behave well under plethysm. By (2-3-1) and (2-4-5), the index set has the structure of a commutative monoid, and so it is preferable to use an index set with a familiar monoid structure. If we were to insist on agreement with Witt's indexing, we would have to replace the sum $m+n$ in (2-3-1) and (2-4-5) with $m+n-(1,1, \ldots)$, where this would be computed in the product group $\mathbb{Z}^{E}$. The reason why this has not come up in earlier work is that the plethysm structure has traditionally been used only through the Frobenius maps $\psi_{\alpha}$. In other words, only the shift operator on the indexing set was used. Thus the distinction between $\mathbb{N}$ and $\mathbb{Z} \geqslant 1$ was not so important because the shift operator $n \mapsto n+1$ is written the same way on both. But making the identification of $\mathbb{N}$ and $\mathbb{Z}_{\geqslant 1}$ a monoid isomorphism would involve the unwelcome addition law $m+n-1$ on $\mathbb{Z} \geqslant 1$.

It is different with the big Witt vectors, where $R$ is $\mathbb{Z}$ and $E$ consists of all maximal ideals 1.15 . They are also traditionally indexed by the positive integers [Hazewinkel 1978, (17.4.4)], but here the positive integers are used multiplicatively rather than additively. In particular, the monoid structure that is required is the obvious one; so the traditional indexing is in agreement with the normalized one: the big Witt ring $W_{p^{n}}(A)$ (using traditional multiplicative indexing) is naturally isomorphic to our $p$-typical ring $W_{n}(A)$ and to Witt's $W_{n+1}^{\prime}(A)$. 
2.6. Localization of the ring $R$ of scalars. Let $R^{\prime}$ be an $E$-flat $R$-algebra such that the structure map $R \rightarrow R^{\prime}$ is an epimorphism of rings, as in 1.20.

Then for each $n \in \mathbb{N}^{(E)}$, we have

$$
\begin{aligned}
R^{\prime} \otimes_{R} \Lambda_{R, E, n} & =R^{\prime} \otimes_{R}\left(\Lambda_{R, E} \cap\left(R[1 / E] \otimes_{R} \Psi_{R, E, n}\right)\right) \\
& \stackrel{\sim}{\rightarrow}\left(R^{\prime} \otimes_{R} \Lambda_{R, E}\right) \cap\left(R^{\prime}[1 / E] \otimes_{R^{\prime}} \Psi_{R^{\prime}, E^{\prime}, n}\right) .
\end{aligned}
$$

(We only need to check that the displayed map is an isomorphism along $E$, in which case it is true because $R^{\prime}$ is $E$-flat over $R$.) By (1-20-1), this gives an isomorphism of $R^{\prime}$-algebras

$$
R^{\prime} \otimes_{R} \Lambda_{R, E, n} \stackrel{\sim}{\longrightarrow} \Lambda_{R^{\prime}, E^{\prime}, n} .
$$

The induced isomorphism of represented functors gives natural maps

$$
W_{R^{\prime}, E^{\prime}, n}\left(A^{\prime}\right) \stackrel{\sim}{\longrightarrow} W_{R, E, n}\left(A^{\prime}\right),
$$

for $R^{\prime}$-algebras $A^{\prime}$. If $A$ is an $R$-algebra, the inverse of this map induces a map

$$
R^{\prime} \otimes_{R} W_{R, E, n}(A) \longrightarrow W_{R^{\prime}, E^{\prime}, n}\left(R^{\prime} \otimes_{R} A\right)
$$

We will see in 6.1 that this is an isomorphism.

As with (1-20-3), the map (2-6-2) induces an isomorphism

$$
\Lambda_{R, E, n} \odot B^{\prime} \stackrel{\sim}{\longrightarrow} \Lambda_{R^{\prime}, E^{\prime}, n} \odot B^{\prime},
$$

for any $R^{\prime}$-algebra $B^{\prime}$,

2.7. Proposition. Let $A$ be an E-flat R-algebra. Then the ghost map

$$
w_{\leqslant n}: W_{R, E, n}(A) \longrightarrow A^{[0, n]}
$$

is injective. If $A$ is an $R[1 / E]$-algebra, it is an isomorphism.

Recall that the analogous facts for infinite-length Witt vectors are also true, either by construction 1.8 or by the universal property 1.9 .

Proof. If every ideal in $E$ is the unit ideal, then $\Lambda_{R, E}=\Psi_{R, E}$, and hence we have $\Lambda_{R, E, n}=\Psi_{R, E, n}$. The statement about $R[1 / E]$-algebras then follows from (2-6-1). The statement about $E$-flat $R$-algebras follows by considering the injection

$$
A \rightarrow R[1 / E] \otimes_{R} A
$$

and applying the previous case to $R[1 / E] \otimes_{R} A$. 


\section{Principal single-prime case}

For this section, we will restrict to the case where $E$ consists of one ideal $\mathfrak{m}$ generated by an element $\pi$. Our purpose is to extend the classical components of Witt vectors from the $p$-typical context (where $R$ is $\mathbb{Z}$ and $E$ consists of the single ideal $p \mathbb{Z}$ ) to this slightly more general one. The reason for this is that the Witt components are well-suited to calculation. In the following sections, we will see how to use them, together with $4.1,5.4$, and 6.1, to draw conclusions when $E$ is general.

In fact, the usual arguments and definitions in the classical theory of Witt vectors carry over as long as one modifies the usual Witt polynomials by replacing every $p$ in an exponent with $q_{\mathfrak{m}}$, and every $p$ in a coefficient with $\pi$. Some things, such as the Verschiebung operator, depend on the choice of $\pi$, and others do not, such as the Verschiebung filtration.

Let $n$ denote an element of $\mathbb{N}$. Let us abbreviate

$$
\Lambda_{\mathfrak{m}}=\Lambda_{R, E}, \quad \Lambda_{\mathfrak{m}, n}=\Lambda_{R, E, n}, \quad W_{\mathfrak{m}}=W_{R, E}, \quad q=q_{\mathfrak{m}}, \quad \psi=\psi_{\mathfrak{m}}
$$

and so on.

3.1. $\theta$ operators. Define elements $\theta_{\pi, 0}, \theta_{\pi, 1}, \ldots$ of

$$
R[1 / \pi] \otimes_{R} \Lambda_{\mathfrak{m}}=R[1 / \pi] \otimes_{R} \Psi_{\mathfrak{m}}
$$

recursively by the generalized Witt polynomials

$$
\psi^{\circ n}=\theta_{\pi, 0}^{q^{n}}+\pi \theta_{\pi, 1}^{q^{n-1}}+\cdots+\pi^{n} \theta_{\pi, n} .
$$

(Note that the exponent on the left side means iterated composition, while the exponents on the right mean usual exponentiation, iterated multiplication.) As in 1.5 , we can view the elements $\theta_{\pi, i}$ as natural operators on $\Psi_{R[1 / \pi], \mathfrak{m}}$-rings. We will often write $\theta_{i}=\theta_{\pi, i}$ when $\pi$ is clear.

3.2. Lemma. We have

$$
\psi \circ \theta_{\pi, n}=\theta_{\pi, n}^{q}+\pi \theta_{\pi, n+1}+\pi P\left(\theta_{\pi, 0}, \ldots, \theta_{\pi, n-1}\right),
$$

for some polynomial $P\left(\theta_{\pi, 0}, \ldots, \theta_{\pi, n-1}\right)$ with coefficients in $R$.

Proof. It is clear for $n=0$. For $n \geqslant 1$, we will use induction. Recall the general implication

$$
x \equiv y \bmod \mathfrak{m} \quad \Longrightarrow \quad x^{q^{j}} \equiv y^{q^{j}} \bmod \mathfrak{m}^{j+1}
$$


for $j \geqslant 1$, which itself is easily proved by induction. Together with the formula (3-2-1) for $\psi \circ \theta_{\pi, i}$ with $i<n$, this implies

$$
\begin{aligned}
\psi \circ \psi^{\circ n} & =\sum_{i=0}^{n} \pi^{i}\left(\psi \circ \theta_{i}\right)^{q^{n-i}} \\
& \equiv \pi^{n} \psi \circ \theta_{n}+\sum_{i=0}^{n-1} \pi^{i}\left(\theta_{i}^{q}\right)^{q^{n-i}} \bmod \mathfrak{m}^{n+1} R\left[\theta_{0}, \ldots, \theta_{n-1}\right]
\end{aligned}
$$

When this is combined with the defining formula (3-1-1) for $\psi^{\circ(n+1)}$, we have

$$
\pi^{n} \psi \circ \theta_{n} \equiv \pi^{n} \theta_{n}^{q}+\pi^{n+1} \theta_{n+1} \bmod \mathfrak{m}^{n+1} R\left[\theta_{0}, \ldots, \theta_{n-1}\right] .
$$

Dividing by $\pi^{n}$ completes the proof.

3.3. Proposition. The elements $\theta_{\pi, 0}, \theta_{\pi, 1}, \ldots$ of $R[1 / \pi] \otimes_{R} \Lambda_{\mathfrak{m}}$ lie in $\Lambda_{\mathfrak{m}}$, and they generate $\Lambda_{\mathfrak{m}}$ freely as an R-algebra. Further, the elements $\theta_{\pi, 0}, \ldots, \theta_{\pi, n}$ lie in $\Lambda_{\mathfrak{m}, n}$, and they generate $\Lambda_{\mathfrak{m}, n}$ freely as an $R$-algebra.

This is essentially [Witt 1937, Theorem 1].

Proof. By induction, the elements $\theta_{0}, \ldots, \theta_{n}$ generate the same sub- $R[1 / \pi]$-algebra of $R[1 / \pi] \otimes_{R} \Lambda_{\mathfrak{m}}$ as $\psi^{\circ 0}, \ldots, \psi^{\circ n}$, and are hence algebraically independent over $R[1 / \pi]$. Since $R \subseteq R[1 / \pi]$, they are also algebraically independent over $R$.

Let $B_{n}$ be the sub- $R$-algebra of $R[1 / \pi] \otimes_{R} \Lambda_{\mathfrak{m}}$ generated by $\theta_{0}, \ldots, \theta_{n}$, and let $B=\bigcup_{n} B_{n}$. To show $\Lambda_{\mathfrak{m}} \supseteq B$, we may assume by induction that $\Lambda_{\mathfrak{m}} \supseteq B_{n}$ and then show $\Lambda_{\mathfrak{m}} \supseteq B_{n+1}$. By 3.2 and because $\Lambda_{\mathfrak{m}}$ is a $\Lambda_{\mathfrak{m}}$-ring, we have

$$
\pi \theta_{n+1} \in\left(\psi \circ \theta_{n}-\theta_{n}^{q}\right)+\mathfrak{m} \Lambda_{\mathfrak{m}, n} \subseteq \mathfrak{m} \Lambda_{\mathfrak{m}}
$$

Dividing by $\pi$, we have $\theta_{n+1} \in \Lambda_{\mathfrak{m}}$, and hence $\Lambda_{\mathfrak{m}} \supseteq B_{n}\left[\theta_{n+1}\right]=B_{n+1}$.

On the other hand, by 3.2 again, we have

$$
\psi \circ \theta_{n} \equiv \theta_{n}^{q} \bmod \mathfrak{m} B_{n+1}
$$

for all $n$. Hence $B$, being generated by the $\theta_{n}$, is a sub- $\Lambda_{\mathfrak{m}}$-ring of $R[1 / \pi] \otimes_{R} \Lambda_{\mathfrak{m}}$. It follows that $B \supseteq \Lambda_{\mathfrak{m}} \circ e=\Lambda_{\mathfrak{m}}$, and therefore $B=\Lambda_{\mathfrak{m}}$.

Last, the equality $\Lambda_{\mathfrak{m}, n}=B_{n}$ follows immediately from the above:

$$
\begin{aligned}
\Lambda_{\mathfrak{m}, n} & =\Lambda_{\mathfrak{m}} \cap\left(R[1 / \pi] \otimes_{R} \Psi_{\mathfrak{m}, n}\right)=B \cap\left(R[1 / \pi] \otimes_{R} \Psi_{\mathfrak{m}, n}\right) \\
& =R\left[\theta_{0}, \ldots\right] \cap R[1 / \pi]\left[\theta_{0}, \ldots, \theta_{n}\right] \\
& =R\left[\theta_{0}, \ldots, \theta_{n}\right]=B_{n} .
\end{aligned}
$$


3.4. Example: Presentations of $\Lambda_{\mathfrak{m}, n} \odot A$. Using 3.3, we can turn a presentation of an $R$-algebra $A$ into a presentation of $\Lambda_{\mathfrak{m}, n} \odot A$. We have

$$
\Lambda_{\mathfrak{m}, n} \odot R[x] \cong \Lambda_{\mathfrak{m}, n}=R\left[\theta_{0}, \ldots, \theta_{n}\right],
$$

where $\theta_{k}$ is short for $\theta_{\pi, k}$, which corresponds to the element $\theta_{\pi, k}(x)=\theta_{\pi, k} \odot x$.

Because the functor $\Lambda_{\mathfrak{m}, n} \odot$ - preserves coproducts and coequalizers, we have

$$
\Lambda_{\mathfrak{m}, n} \odot\left(R\left[x_{1}, \ldots, x_{r}\right] /\left(f_{1}, \ldots, f_{s}\right)\right)=R\left[\theta_{i}\left(x_{j}\right)\right] /\left(\theta_{i}\left(f_{k}\right)\right),
$$

where $0 \leqslant i \leqslant n, 1 \leqslant j \leqslant r$, and $1 \leqslant k \leqslant s$. Here each expression $\theta_{i}\left(x_{j}\right)$ is a single free variable, and $\theta_{i}\left(f_{k}\right)$ is understood to be the polynomial in the variables $\theta_{i}\left(x_{j}\right)$ that results from expanding $\theta_{i}\left(f_{k}\right)$ using the sum and product laws for $\theta_{i}$. Because $\Lambda_{\mathfrak{m}, n} \odot$ - preserves filtered colimits, we can give a similar presentation of $\Lambda_{\mathfrak{m}, n} \odot A$ for any $R$-algebra $A$. Similarly, we can take the colimit over $n$ to get a presentation for $\Lambda_{\mathfrak{m}} \odot A$.

In the $E$-typical case, where $E$ is finite, one can write down a presentation of $\Lambda_{R, E} \odot A$ by iterating (3-4-1), according to 5.3 below. We can pass from the case where $E$ is finite to the case where it is arbitrary by taking colimits, as in 5.1.

The method above is not particular to the $\theta$ operators-it works for any subset of $\Lambda_{\mathfrak{m}, n}$ that generates it freely as an $R$-algebra. For example, we can use the $\delta$ operators of 1.19. Let $\delta^{i} \in \Lambda_{\mathfrak{m}}$ denote the $i$-th iterate of $\delta_{\pi}$. Then the elements $\delta^{0}, \ldots, \delta^{n}$ lie in $\Lambda_{\mathfrak{m}, n}$ and freely generate it as an $R$-algebra. (As in 3.3, this follows by induction, but in this case, there are no subtle congruences to check.) Therefore we have

$$
\Lambda_{\mathfrak{m}, n} \odot\left(R\left[x_{1}, \ldots, x_{r}\right] /\left(f_{1}, \ldots, f_{s}\right)\right)=R\left[\delta^{i}\left(x_{j}\right)\right] /\left(\delta^{i}\left(f_{k}\right)\right),
$$

where $0 \leqslant i \leqslant n, 1 \leqslant j \leqslant r$, and $1 \leqslant k \leqslant s$. We interpret the expressions $\delta^{i}\left(x_{j}\right)$ and $\delta^{i}\left(f_{k}\right)$ as above. The general $E$-typical case can be handled as above. (See [Buium and Simanca 2009, proof of Proposition 2.12].)

3.5. Witt components. It follows from 3.3 that, given $\pi$, we have a bijection

$$
W_{\mathfrak{m}}(A) \stackrel{\sim}{\longrightarrow} A \times A \times \cdots,
$$

which sends a map $f: \Lambda_{\mathfrak{m}} \rightarrow A$ to the sequence $\left(f\left(\theta_{\pi, 0}\right), f\left(\theta_{\pi, 1}\right), \ldots\right)$. To make the dependence on $\pi$ explicit, we will often write $\left(x_{0}, x_{1}, \ldots\right)_{\pi}$ for the image of $\left(x_{0}, x_{1}, \ldots\right)$ under the inverse of this map. If $R=\mathbb{Z}$ and $\pi=p$, then this identifies $W_{\mathfrak{m}}(A)$ with the ring of $p$-typical Witt vectors as defined traditionally. Similarly, when $R$ is a complete discrete valuation ring, we get an identification of $W_{\mathfrak{m}}(A)$ with Hazewinkel's ring of ramified Witt vectors $W_{q, \infty}^{R}(A)$. (See [Hazewinkel 1978, (18.6.13), (25.3.17), and (25.3.26)(i)].) We call the $x_{i}$ the Witt components (relative to $\pi)$ of the element $\left(x_{0}, \ldots\right)_{\pi} \in W(A)$. 
Similarly, using the free generating set $\theta_{\pi, 0}, \ldots, \theta_{\pi, n}$ of $\Lambda_{\mathfrak{m}, n}$, we have a bijection

$$
W_{\mathfrak{m}, n}(A) \stackrel{\sim}{\longrightarrow} A^{[0, n]} .
$$

As above, we will write $\left(x_{0}, \ldots, x_{n}\right)_{\pi}$ for the image of $\left(x_{0}, \ldots, x_{n}\right)$ under the inverse of this map. This identifies $W_{\mathfrak{m}, n}(A)$ with the traditionally defined ring of $p$-typical Witt vectors of length $n+1$. (For remarks on the +1 shift, see 2.5.)

Note that the Witt components do not depend on the choice of $\pi$ in a simple, multilinear way. For example, if $u$ is an invertible element of $R$ and we have

$$
\left(x_{0}, x_{1}, \ldots\right)_{\pi}=\left(y_{0}, y_{1}, \ldots\right)_{u \pi}
$$

then we have

$$
x_{0}=y_{0}, \quad x_{1}=u y_{1}, \quad x_{2}=u^{2} y_{2}+\pi^{-1}\left(u-u^{q}\right) y_{1}^{q}, \quad \ldots
$$

As in 3.4, we could use the free generating set $\delta^{0}, \delta^{1}, \ldots$ of $\Lambda_{\mathfrak{m}}$ instead of $\theta_{0}, \theta_{1}, \ldots$ This would give a different bijection between $W_{\mathfrak{m}}(A)$ and the set $A^{\mathbb{N}}$, and hence an $R$-algebra structure on the set $A^{\mathbb{N}}$ which is isomorphic to Witt's but not equal to it. The truncated versions agree up to $A \times A$, but differ after that. This is simply because $\delta^{0}=\theta_{0}$ and $\delta^{1}=\theta_{1}$, but $\delta^{2} \neq \theta_{2}$. (See [Joyal 1985b, p. 179].)

3.6. The ghost principle. It follows from the descriptions (3-5-1) and (3-5-2) that $W_{\mathfrak{m}}$ and $W_{\mathfrak{m}, n}$ preserve surjectivity. On the other hand, every $R$-algebra is a quotient of an $\mathfrak{m}$-flat $R$-algebra (even a free one). Therefore to prove any functorial identity involving rings of Witt vectors when $\mathfrak{m}$ is principal, it is enough to restrict to the $\mathfrak{m}$-flat case. Further, any $\mathfrak{m}$-flat $R$-algebra $A$ is contained in an $R[1 / \mathfrak{m}]-$ algebra, such as $R[1 / \mathfrak{m}] \otimes_{R} A$. Since $W_{\mathfrak{m}}$ and $W_{\mathfrak{m}, n}$, being representable functors, preserve injectivity, it is even enough to check functorial identities on $R[1 / \mathfrak{m}]$ algebras $A$, in which case rings of Witt vectors agree with the much more tractable rings of ghost components. An example with details is given in 3.7.

3.7. Verschiebung. For any $R$-algebra $A$ define an operator $V_{\pi}$, called the Verschiebung (relative to $\pi$ ), on $W_{\mathfrak{m}}(A)$ by

$$
V_{\pi}\left(\left(y_{0}, y_{1}, \ldots\right)_{\pi}\right)=\left(0, y_{0}, y_{1}, \ldots\right)_{\pi} .
$$

This is clearly functorial in $A$. Define another, identically denoted operator on the ghost ring $A^{\mathbb{N}}$ by the formula

$$
V_{\pi}\left(\left\langle z_{0}, z_{1}, \ldots\right\rangle\right)=\left\langle 0, \pi z_{0}, \pi z_{1}, \ldots\right\rangle .
$$

These operators are compatible in that we have $w\left(V_{\pi}(y)\right)=V_{\pi}(w(y))$ for all $y \in W_{\mathfrak{m}}(A)$, and the operator $V_{\pi}$ on the ghost ring is clearly $R$-linear. It follows by 
the ghost principle that the operator $V_{\pi}$ on $W_{\mathfrak{m}}(A)$ is $R$-linear. Here is the argument in some detail.

We need to check the identities $r V_{\pi}(y)=V_{\pi}(r y)$ and $V_{\pi}(x+y)=V_{\pi}(x)+V_{\pi}(y)$, for $r \in R, x, y \in W_{\mathfrak{m}}(A)$. Write $x=\left(x_{0}, x_{1}, \ldots\right)_{\pi}$ and $y=\left(y_{0}, y_{1}, \ldots\right)_{\pi}$. If $A$ is a $E$-flat, the ghost map $w: W_{\mathfrak{m}}(A) \rightarrow A^{\mathbb{N}}$ is injective. Therefore $V_{\pi}$ is $R$-linear on $W_{\mathfrak{m}}(A)$, by the $R$-linearity of $V_{\pi}$ on the ghost ring.

The general case then follows from $E$-flat case. Fix an $E$-flat $R$-algebra $\tilde{A}$ with a surjective $R$-algebra map $\tilde{A} \rightarrow A$. For each $i$, let $\tilde{y}_{i}$ be a pre-image of $y_{i}$, and set $\tilde{y}=\left(\tilde{y}_{0}, \ldots\right)_{\pi} \in W_{\mathfrak{m}}(A)$. The induced map $f: W_{\mathfrak{m}}(\tilde{A}) \rightarrow W_{\mathfrak{m}}(A)$ then satisfies $f(\tilde{y})=y$. Therefore we have

$$
\begin{aligned}
V_{\pi}(r y) & =V_{\pi}(r f(\tilde{y}))=V_{\pi}(f(r \tilde{y}))=f\left(V_{\pi}(r \tilde{y})\right) \\
& =f\left(r V_{\pi}(\tilde{y})\right)=r f\left(V_{\pi}(\tilde{y})\right)=r V_{\pi}(f(\tilde{y}))=r V_{\pi}(y) .
\end{aligned}
$$

The additivity axiom follows similarly.

3.8. Example. $W_{R, \mathfrak{m}, n}(R)$ has a presentation

$$
R\left[x_{1}, \ldots, x_{n}\right] /\left(x_{i} x_{j}-\pi^{i} x_{j} \mid 1 \leqslant i \leqslant j \leqslant n\right),
$$

where the element $x_{i}$ corresponds to $V_{\pi}^{i}(1)$.

3.9. Teichmüller lifts. Under the composition

$$
A \stackrel{a \mapsto[a]}{\longrightarrow} W(A) \stackrel{w}{\longrightarrow} A \times A \times \cdots
$$

(see 1.21), the image of $a$ is $\left\langle a, a^{q}, a^{q^{2}}, \ldots\right\rangle$. It follows from the ghost principle that

$$
[a]=(a, 0,0, \ldots)_{\pi} \in W(A) .
$$

Multiplication by Teichmüller lifts also has a simple description in terms of Witt components:

$$
[a]\left(\ldots, b_{i}, \ldots\right)_{\pi}=\left(\ldots, a^{q^{i}} b_{i}, \ldots\right)_{\pi} .
$$

Again, this follows from the ghost principle.

\section{General single-prime case}

Assume $E$ consists of a single ideal $\mathfrak{m}$, possibly not principal. Let $n$ be an element of $\mathbb{N}$. Let us write $W_{R, \mathfrak{m}, n}=W_{R, E, n}$ and so on.

Let $K_{\mathfrak{m}}$ denote $R_{\mathfrak{m}}[1 / \mathfrak{m}]$. If $\mathfrak{m}$ is the unit ideal, we understand $R_{\mathfrak{m}}$, and hence $K_{\mathfrak{m}}$, to be the zero ring. Otherwise, $R_{\mathfrak{m}}$ is a discrete valuation ring and $K_{\mathfrak{m}}$ is its fraction field. In particular, $\mathfrak{m}$ becomes principal in $R[1 / \mathfrak{m}], R_{\mathfrak{m}}$, and $K_{\mathfrak{m}}$. The following proposition then allows us to describe $W_{R, \mathfrak{m}, n}(A)$ in terms of the case where $\mathfrak{m}$ is principal, and hence in terms of Witt components. 
4.1. Proposition. For $R^{\prime}=R[1 / \mathfrak{m}], R_{\mathfrak{m}}, K_{\mathfrak{m}}$, write $W_{R^{\prime}, \mathfrak{m}, n}=W_{R^{\prime}, \mathfrak{m} R^{\prime}, n}$. Then for any $R$-algebra $A$, the ring $W_{R, \mathfrak{m}, n}(A)$ is the equalizer of the two maps

$$
W_{R[1 / \mathfrak{m}], \mathfrak{m}, n}\left(R[1 / \mathfrak{m}] \otimes_{R} A\right) \times W_{R_{\mathfrak{m}}, \mathfrak{m}, n}\left(R_{\mathfrak{m}} \otimes_{R} A\right) \longrightarrow W_{K_{\mathfrak{m}}, \mathfrak{m}, n}\left(K_{\mathfrak{m}} \otimes_{R} A\right)
$$

induced by projection onto the two factors and the bifunctoriality of $W_{-, \mathfrak{m}, n}(-)$.

Proof. The diagram

$$
R \longrightarrow R[1 / \mathfrak{m}] \times R_{\mathfrak{m}} \underset{\mathrm{pr}_{2}}{\stackrel{\mathrm{pr}_{1}}{\longrightarrow}} K_{\mathfrak{m}}
$$

is an equalizer diagram. Since $K_{\mathfrak{m}}$ is $\mathfrak{m}$-flat, so is any sub-R-module of $K_{\mathfrak{m}}$. It follows that for any $R$-algebra $A$, the induced diagram

$$
A \longrightarrow\left(R[1 / \mathfrak{m}] \times R_{\mathfrak{m}}\right) \otimes_{R} A \longrightarrow K_{\mathfrak{m}} \otimes_{R} A
$$

is an equalizer diagram. Since $W_{R, \mathfrak{m}, n}$ is representable, it preserves equalizers, and so the induced diagram (writing $W_{n}=W_{R, \mathfrak{m}, n}$ )

$$
W_{n}(A) \longrightarrow W_{n}\left(R[1 / \mathfrak{m}] \otimes_{R} A\right) \times W_{n}\left(R_{\mathfrak{m}} \otimes_{R} A\right) \longrightarrow W_{n}\left(K_{\mathfrak{m}} \otimes_{R} A\right)
$$

is also an equalizer diagram. Then (2-6-2) completes the proof.

4.2. Verschiebung in general. We can define Verschiebung maps

$$
V^{j}: \mathfrak{m}^{j} \otimes_{R} W_{R, \mathfrak{m}}(A) \longrightarrow W_{R, \mathfrak{m}}(A) .
$$

To do this, it is enough, by 4.1 , to restrict to the case where $\mathfrak{m}$ is principal, as long as our construction is functorial in $A$ and $R$. So, choose a generator $\pi \in \mathfrak{m}$ and define

$$
V^{j}\left(\pi^{j} \otimes y\right)=V_{\pi}^{j}(y),
$$

for all $y \in W_{R, \mathfrak{m}}(A)$. On ghost components it satisfies

$$
V^{j}\left(x \otimes\left\langle z_{0}, z_{1}, \ldots\right\rangle\right)=\left\langle 0, \ldots, 0, x z_{0}, x z_{1}, \ldots\right\rangle,
$$

where the number of leading zeros is $j$. In particular, it is independent of the choice of $\pi$, by the ghost principle.

If we write $W_{R, \mathfrak{m}}(A)_{(j)}$ for $W_{R, \mathfrak{m}}(A)$, viewed as a $W_{R, \mathfrak{m}}(A)$-algebra by way of the map $\psi_{j}: W_{R, \mathfrak{m}}(A) \rightarrow W_{R, \mathfrak{m}}(A)$, then the map

$$
V^{j}: \mathfrak{m}^{j} \otimes_{R} W_{R, \mathfrak{m}}(A)_{(j)} \longrightarrow W_{R, \mathfrak{m}}(A),
$$

is $W_{R, \mathfrak{m}}(A)$-linear, as is easily checked using the ghost principle. Expressed as a formula, it says

$$
V^{j}\left(x \otimes y \psi_{j}(z)\right)=V^{j}(x \otimes y) z .
$$


In particular, the image $V^{j} W_{R, \mathfrak{m}}(A)$ of $V^{j}$ is an ideal of $W_{R, \mathfrak{m}}(A)$.

Let us also record the identities

$$
\psi_{j}\left(V^{j}(x \otimes y)\right)=x y
$$

and

$$
V^{j}(x \otimes y) V^{j}\left(x^{\prime} \otimes y^{\prime}\right)=x V^{j}\left(x^{\prime} \otimes y y^{\prime}\right) \in \mathfrak{m}^{j} V^{j} W_{R, \mathfrak{m}}(A) .
$$

Again, one checks these using the ghost principle.

Finally, for any $n \in \mathbb{N}$, the map $V^{j}$ descends to a map

$$
V^{j}: \mathfrak{m}^{j} \otimes_{R} W_{R, \mathfrak{m}, n}(A)_{(j)} \longrightarrow W_{R, \mathfrak{m}, n+j}(A),
$$

and the obvious analogues of the identities above hold here.

4.3. Remark. We can define Verschiebung maps even if we no longer assume there is only one ideal in $E$. For any $j \in \mathbb{N}^{(E)}$, let $J$ denote the ideal $\prod_{\alpha} \mathfrak{m}_{\alpha}^{j_{\alpha}}$ of $R$. Then $V^{j}$ would be a map $J \otimes_{R} W_{R, E}(A) \rightarrow W_{R, E}(A)$. The identities above, suitably interpreted, continue to hold. We will not need this multiple-prime version.

\subsection{Proposition. The sequence}

$$
0 \longrightarrow \mathfrak{m}^{j} \otimes_{R} W_{R, \mathfrak{m}, n}(A)_{(j)} \stackrel{V^{j}}{\longrightarrow} W_{R, \mathfrak{m}, n+j}(A) \longrightarrow W_{R, \mathfrak{m}, j}(A) \longrightarrow 0
$$

is exact.

Proof. Write $W_{R^{\prime}, n}=W_{R^{\prime}, \mathfrak{m} R^{\prime}, n}$ when $R^{\prime}$ is an $R$-algebra such that the ideal $\mathfrak{m} R^{\prime}$ is supramaximal.

First consider the case where $\mathfrak{m}$ is principal. Let $\pi \in \mathfrak{m}$ be a generator. Using (3-7-1), it is clear that $V^{j}$ is injective and that its image is the set of Witt vectors whose Witt components (relative $\pi$ ) are 0 in positions 0 to $j-1$. By 3.5, the preimage of 0 under the map $W_{R, n+j}(A) \rightarrow W_{R, j}(A)$ is the same subset, and the map $W_{R, n+j}(A) \rightarrow W_{R, j}(A)$ is surjective.

Now consider the general case. Augment the diagram (4-4-1) by expressing each term of (4-4-1) as an equalizer as in 4.1. Here we use that $\mathfrak{m}$ is $R$-flat. It then follows from the principal case and the snake lemma that (4-4-1) is left exact.

It remains to prove that the map $W_{R, n+j}(A) \rightarrow W_{R, j}(A)$ is surjective. By induction, we can assume $n=1$. By 4.1 , for any $i \in \mathbb{N}$ we have

$$
W_{R, i}(A)=W_{R_{\mathfrak{m}}, i}\left(R_{\mathfrak{m}} \otimes_{R} A\right) \times_{W_{K_{\mathfrak{m}}, i}\left(K_{\mathfrak{m}} \otimes_{R} A\right)} W_{R[1 / \mathfrak{m}], i}\left(R[1 / \mathfrak{m}] \otimes_{R} A\right) .
$$

Now let $\pi$ denote a generator of the maximal ideal of $R_{\mathfrak{m}}$, and suppose two elements

$$
\begin{aligned}
& y=\left(y_{0}, \ldots, y_{j}\right)_{\pi} \in W_{R_{\mathfrak{m}}, j}\left(R_{\mathfrak{m}} \otimes_{R} A\right), \\
& z=\left\langle z_{0}, \ldots, z_{j}\right\rangle \in\left(R[1 / \mathfrak{m}] \otimes_{R} A\right)^{j+1}=W_{R[1 / \mathfrak{m}], j}\left(R[1 / \mathfrak{m}] \otimes_{R} A\right)
\end{aligned}
$$


have the same image in $W_{K_{\mathfrak{m}}, j}\left(K_{\mathfrak{m}} \otimes_{R} A\right)$. To lift the corresponding element of $W_{j}(A)$ to $W_{j+1}(A)$, we need to find elements

$$
y_{j+1} \in R_{\mathfrak{m}} \otimes_{R} A \quad \text { and } \quad z_{j+1} \in R[1 / \mathfrak{m}] \otimes_{R} A
$$

such that in $K_{\mathfrak{m}} \otimes_{R} A$, we have

$$
y_{0}^{q^{j+1}}+\cdots+\pi^{j+1} y_{j+1}=z_{j+1} .
$$

So, choose an element $z_{j+1} \in A$ whose image under the surjection

$$
A \longrightarrow A /(\mathfrak{m} A)^{j+1}=R_{\mathfrak{m}} /\left(\mathfrak{m} R_{\mathfrak{m}}\right)^{j+1} \otimes_{R} A
$$

agrees with the image of $y_{0}^{q^{j+1}}+\cdots+\pi^{j} y_{j}$. It follows that the element

$$
y_{0}^{q^{j+1}}+\cdots+\pi^{j} y_{j}^{q}-1 \otimes z_{j+1} \in R_{\mathfrak{m}} \otimes_{R} A
$$

lies in $\pi^{j+1}\left(R_{\mathfrak{m}} \otimes_{R} A\right)$. It thus equals $\pi^{j+1} y_{j+1}$ for some element $y_{j+1} \in R_{\mathfrak{m}} \otimes_{R} A$. And so $y_{j+1}$ and $z_{j+1}$ satisfy (4-4-2).

4.5. Corollary. For any R-algebra A, we have

$$
\bigoplus_{i \in[0, n]} \mathfrak{m}^{i} \otimes_{R} A_{(i)} \stackrel{\sim}{\longrightarrow} \operatorname{gr}_{V} W_{R, \mathfrak{m}, n}(A)
$$

where $A_{(i)}$ denotes $A$ viewed as a $W_{n}(A)$-module via the ring map $w_{i}: W_{n}(A) \rightarrow$ A.

4.6. Reduced ghost components. We can define infinitely many ghost components for Witt vectors of finite length $n$ if we are willing to settle for answers modulo $\mathfrak{m}^{n+1}$.

First assume $\mathfrak{m}$ is generated by some element $\pi$. By examining the Witt polynomials (3-1-1), we can see that for any $i \geqslant 0$, the composition

$$
W_{R, \mathfrak{m}}(A) \stackrel{w_{i}}{\longrightarrow} A \longrightarrow A / \mathfrak{m}^{n+1} A
$$

vanishes on $V^{n+1} W_{R, \mathfrak{m}}(A)$. It therefore factors through $W_{R, \mathfrak{m}, n}(A)$, giving a map $\bar{w}_{i}$ from $W_{R, \mathfrak{m}, n}(A)$ to $A / \mathfrak{m}^{n+1} A$.

When $\mathfrak{m}$ is not assumed to be principal, we define $\bar{w}_{i}$ by localizing at $\mathfrak{m}$ : $W_{R, \mathfrak{m}, n}(A) \rightarrow W_{R_{\mathfrak{m}}, \mathfrak{m} R_{\mathfrak{m}}, n}\left(R_{\mathfrak{m}} \otimes_{R} A\right) \stackrel{\bar{w}_{i}}{\longrightarrow}\left(R_{\mathfrak{m}} \otimes_{R} A\right) / \mathfrak{m}^{n+1}\left(R_{\mathfrak{m}} \otimes_{R} A\right)=A / \mathfrak{m}^{n+1} A$, where the middle map is $\bar{w}_{i}$ as constructed above in the principal case. We call the composition

$$
W_{R, \mathfrak{m}, n}(A) \stackrel{\bar{w}_{i}}{\longrightarrow} A / \mathfrak{m}^{n+1} A
$$

the $i$-th reduced ghost component map. 


\section{Multiple-prime case}

The purpose of this section is to give some results on reducing the family $E$ (of 1.2) to simpler families. The first reduces from the case where $E$ is arbitrary to the case where it is finite, and the second reduces from the case where it is finite to the case where it has a single element. We will often write $W_{E}=W_{R, E}, \Lambda_{E}=\Lambda_{R, E}$, and so on, for short.

\subsection{Proposition. The canonical maps}

$$
\begin{aligned}
\operatorname{colim}_{E^{\prime}} \Lambda_{R, E^{\prime}} & \longrightarrow \Lambda_{R, E}, \\
\operatorname{colim}_{E^{\prime}} \Lambda_{R, E^{\prime}, n^{\prime}} & \longrightarrow \Lambda_{R, E, n}
\end{aligned}
$$

are isomorphisms. Here $E^{\prime}$ runs over the finite subfamilies of $E$, and $n^{\prime}$ is the restriction to $E^{\prime}$ of a given element $n \in \mathbb{N}^{(E)}$.

Proof. Consider (5-1-1) first. Since each map $\Lambda_{E^{\prime}} \rightarrow \Lambda_{E}$ is an injection, (5-1-1) is an injection. Therefore, since $\Lambda_{E}$ is freely generated as a $\Lambda_{E}$-ring by the element $e=\psi_{0}$, it is enough to show the sub- $\Psi_{E}$-ring $\operatorname{colim}_{E^{\prime}} \Lambda_{E^{\prime}}$ of $\Lambda_{E}$ is a sub- $\Lambda_{E}$-ring. Since it is flat, we only need to check the Frobenius lift property. So, suppose $\mathfrak{m} \in E$. For any element $x$ of the colimit, there is a finite family $E^{\prime \prime}$ such that $x \in \Lambda_{E^{\prime \prime}}$ and $\mathfrak{m} \in E^{\prime \prime}$. But $\Lambda_{E^{\prime \prime}}$ is a $\Lambda_{E^{\prime \prime}}$-ring. So we have $\psi_{\mathfrak{m}}(x) \equiv x^{q_{\mathfrak{m}}}$ modulo $\mathfrak{m} \Lambda_{E^{\prime \prime}}$, and hence modulo $\mathfrak{m}\left(\operatorname{colim}_{E^{\prime}} \Lambda_{E^{\prime}}\right)$. Therefore the Frobenius lift property holds for the colimit ring.

Then (5-1-2) follows:

$$
\begin{aligned}
\Lambda_{E, n} & =\left(R[1 / E] \otimes_{R} \Psi_{E, n}\right) \cap \Lambda_{E}=\left(\operatorname{colim}_{E^{\prime}} R[1 / E] \otimes_{R} \Psi_{E^{\prime}, n^{\prime}}\right) \cap \operatorname{colim}_{E^{\prime}} \Lambda_{E^{\prime}} \\
& =\operatorname{colim}_{E^{\prime}}\left(\left(R[1 / E] \otimes_{R} \Psi_{E^{\prime}, n^{\prime}}\right) \cap \Lambda_{E^{\prime}}\right)=\operatorname{colim}_{E^{\prime}} \Lambda_{E^{\prime}, n^{\prime}}
\end{aligned}
$$

5.2. Corollary. For any $R$-algebra A, the canonical maps

$$
\begin{gathered}
W_{R, E}(A) \longrightarrow \lim _{E^{\prime}} W_{R, E^{\prime}}(A), \\
W_{R, E, n}(A) \longrightarrow \lim _{E^{\prime}} W_{R, E^{\prime}, n^{\prime}}(A)
\end{gathered}
$$

are isomorphisms, where $E^{\prime}, n$, and $n^{\prime}$ are as in 5.1.

5.3. Proposition. Let $E^{\prime} \sqcup E^{\prime \prime}$ be a partition of $E$. Then the canonical maps

$$
\begin{aligned}
\Lambda_{R, E^{\prime}} \odot_{R} \Lambda_{R, E^{\prime \prime}} & \longrightarrow \Lambda_{R, E}, \\
\Lambda_{R, E^{\prime}, n^{\prime}} \odot_{R} \Lambda_{R, E^{\prime \prime}, n^{\prime \prime}} & \longrightarrow \Lambda_{R, E, n}
\end{aligned}
$$

are isomorphisms, where $n^{\prime}$ and $n^{\prime \prime}$ denote the restrictions to $E^{\prime}$ and $E^{\prime \prime}$ of a given element $n \in \mathbb{N}^{(E)}$. 
Proof. It is enough to show each map becomes an isomorphism after base change to $R\left[1 / E^{\prime}\right]$ and $R\left[1 / E^{\prime \prime}\right]$. So, by (1-20-1), we can assume every element in either $E^{\prime}$ or $E^{\prime \prime}$ is the unit ideal.

In the second case, we have

$$
\Lambda_{E^{\prime}} \odot_{R} \Lambda_{E^{\prime \prime}}=\Lambda_{E^{\prime}} \odot_{R} R\left[\mathbb{N}^{\left(E^{\prime \prime}\right)}\right]=\Lambda_{E^{\prime}}\left[\mathbb{N}^{\left(E^{\prime \prime}\right)}\right]=\Lambda_{E}
$$

The argument for (5-3-2) is the same, but we replace the generating set $\mathbb{N}^{\left(E^{\prime \prime}\right)}$ with $\left[0, n^{\prime \prime}\right]$.

Now suppose every element in $E^{\prime}$ is the unit ideal. Then a $\Lambda_{E^{\prime}}$-ring is the same as a $\Psi_{E^{\prime}}$-ring. So we have

$$
\Lambda_{E^{\prime}} \odot_{R} \Lambda_{E^{\prime \prime}}=\Lambda_{E^{\prime \prime}}\left[\mathbb{N}^{\left(E^{\prime}\right)}\right]=\Lambda_{E} .
$$

For (5-3-2), replace $\mathbb{N}^{\left(E^{\prime}\right)}$ with $\left[0, n^{\prime}\right]$, as above.

5.4. Corollary. Let $E^{\prime} \sqcup E^{\prime \prime}$ be a partition of $E$. Then for any $R$-algebra $A$, the canonical maps

$$
\begin{gathered}
W_{R, E}(A) \longrightarrow W_{R, E^{\prime \prime}}\left(W_{R, E^{\prime}}(A)\right), \\
W_{R, E, n}(A) \longrightarrow W_{R, E^{\prime \prime}, n^{\prime \prime}}\left(W_{R, E^{\prime}, n^{\prime}}(A)\right)
\end{gathered}
$$

are isomorphisms, where $n, n^{\prime}, n^{\prime \prime}$ are as in 5.3.

5.5. Remark. By the results above, it is safe to say that expressions such as

$$
\Lambda_{\mathfrak{m}_{1}} \odot_{R} \cdots \odot_{R} \Lambda_{\mathfrak{m}_{r}} \quad \text { and } \quad W_{\mathfrak{m}_{r}} \circ \cdots \circ W_{\mathfrak{m}_{1}}(A)
$$

are independent of the ordering of the $\mathfrak{m}_{i}$, assuming the $\mathfrak{m}_{i}$ are pairwise coprime. (Note that it is not generally true that $P \odot P^{\prime} \cong P^{\prime} \odot P$ for plethories $P$ and $P^{\prime}$. See [Borger and Wieland 2005, 2.8].)

If we ask that the expressions in (5-5-1) be independent only up to isomorphism, then it is not even necessary that the $\mathfrak{m}_{\alpha} \in E$ be pairwise coprime 1.2. But invariance up to isomorphism is not a such a useful property, and most of the time coprimality really is necessary. For example, we could look at rings with more than one Frobenius lift at a single maximal ideal, but we would not be able to reduce to the case of a single Frobenius lift. Indeed, if $E$ consists of a single maximal ideal $\mathfrak{m}$, the two endomorphisms $\psi_{W W(A)}$ and $W\left(\psi_{W(A)}\right)$ of $W W(A)$ commute, and the first is clearly a Frobenius lift, but the second is generally not. Therefore $W W(A)$ cannot be the cofree ring with two commuting Frobenius lifts at $\mathfrak{m}$.

In fact, I believe this is the only place where we use the coprimality assumption directly. The rest of our results depend on it only through 5.3. Although I know of no applications, it would be interesting to know whether the abstract setup of this paper, and then the main results, hold when we allow more than one Frobenius lift at each maximal ideal. 


\section{Basic affine properties}

This section provides some basic results about the commutative algebra of Witt vectors. They are just the ones needed to be able to prove the main theorems in sections 8 and 9 and to set up the global theory in the companion paper [Borger 2010]. There are other basic results that could have been included here, but which I have put off to the other paper, where they will be proved for all algebraic spaces.

We continue with the notation of 1.2. Fix an element $n \in \mathbb{N}^{(E)}$. We will often write $W_{n}=W_{E, n}=W_{R, E, n}$ and so on, for short. By 5.2, we may assume that $E$ agrees with the support of $n$, and in particular that it is finite.

6.1. Proposition. Let $R^{\prime}$ be an $E$-flat $R$-algebra such that the structure map $R \rightarrow$ $R^{\prime}$ is a ring epimorphism (as in 1.20). Then the composition

$$
R^{\prime} \otimes_{R} W_{R, E, n}(A) \stackrel{(2-6-3)}{\longrightarrow} W_{R, E, n}\left(R^{\prime} \otimes_{R} A\right) \stackrel{(2-6-2)^{-1}}{\sim} W_{R^{\prime}, E^{\prime}, n}\left(R^{\prime} \otimes_{R} A\right)
$$

is an isomorphism, where $E^{\prime}$ is as in 1.20.

Proof. We may assume by 5.4 that $E$ consists of a single ideal $\mathfrak{m}$. Using 4.1 and the flatness of $R^{\prime}$ over $R$, we are reduced to showing that the functors $W_{R[1 / \mathfrak{m}], \mathfrak{m}, n}$, $W_{R_{\mathfrak{m}}, \mathfrak{m}, n}$, and $W_{K_{\mathfrak{m}}, \mathfrak{m}, n}$ commute with the functor $R^{\prime} \otimes_{R}-$. Therefore we may assume that the ideal $\mathfrak{m}$ is principal.

Write $W_{n}=W_{R, \mathfrak{m}, n}$. The result is clear for $n=0$, because $W_{0}$ is the identity functor. So assume $n \geqslant 1$. By 4.4, we have the map of exact sequences

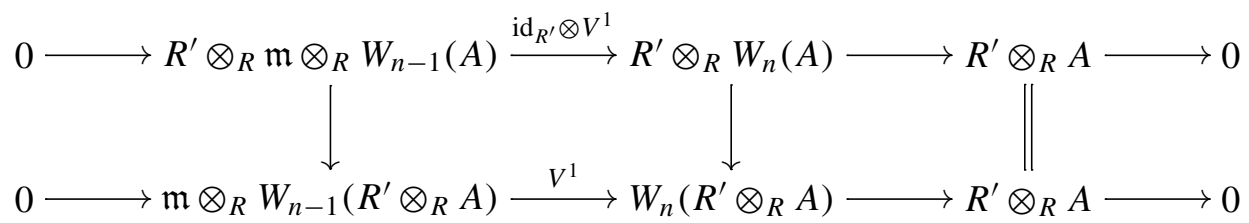

where the vertical maps are given by (2-6-3). By induction the leftmost vertical arrow is an isomorphism. Therefore the inner one is, too.

6.2. Proposition. For any ideal $I$ in an $R$-algebra $A$, let $W_{R, E, n}(I)$ denote the kernel of the canonical map $W_{R, E, n}(A) \rightarrow W_{R, E, n}(A / I)$. Then we have

$$
W_{R, E, n}(I) W_{R, E, n}(J) \subseteq W_{R, E, n}(I J)
$$

for any ideals $I, J$ in $A$.

Proof. We first show that we may assume $E$ consists of a single ideal $\mathfrak{m}$. In doing this, it will be convenient to prove an equivalent form of the statement: if $I J \subseteq K$, where $K$ is an ideal in $A$, then $W_{n}(I) W_{n}(J) \subseteq W_{n}(K)$. Suppose $E=E^{\prime} \amalg\{\mathfrak{m}\}$. Let $n^{\prime}$ be the restriction of $n$ to $E$. Let $I^{\prime}=W_{E^{\prime}, n^{\prime}}(I), J^{\prime}=$ $W_{E^{\prime}, n^{\prime}}(J)$, and $K^{\prime}=W_{E^{\prime}, n^{\prime}}(K)$. By 5.4, we have $W_{E, n}=W_{\mathfrak{m}, n_{\mathfrak{m}}} \circ W_{E^{\prime}, n^{\prime}}$, and 
hence $W_{E, n}(I)=W_{\mathfrak{m}, n_{\mathfrak{m}}}\left(I^{\prime}\right)$ and so on. By induction, we have $I^{\prime} J^{\prime} \subseteq K^{\prime}$, and then applying the result in the single-ideal case gives

$$
W_{E, n}(I) W_{E, n}(J)=W_{\mathfrak{m}, n_{\mathfrak{m}}}\left(I^{\prime}\right) W_{\mathfrak{m}, n_{\mathfrak{m}}}\left(J^{\prime}\right)=W_{\mathfrak{m}, n_{\mathfrak{m}}}\left(K^{\prime}\right)=W_{E, n}(K) .
$$

So we will assume $E=\{\mathfrak{m}\}$ and drop $E$ from the notation.

By 6.1, the statement is Zariski local on $R$, and so we may assume the ideal $\mathfrak{m}$ is generated by some element $\pi$. We will work with Witt components relative to $\pi$.

We need to show that for any elements $x=\left(x_{0}, \ldots, x_{n}\right)_{\pi} \in W_{n}(I)$ and $y=$ $\left(y_{0}, \ldots, y_{n}\right)_{\pi} \in W_{n}(J)$, the product $x y$ is in $W_{n}(I J)$. So it is sufficient to show this in the universal case, where $A$ is the free polynomial algebra $R\left[x_{0}, y_{0}, \ldots, x_{n}, y_{n}\right]$, $I$ is the ideal $\left(x_{0}, \ldots, x_{n}\right)$, and $J$ is the ideal $\left(y_{0}, \ldots, y_{n}\right)$.

Consider the commutative diagram

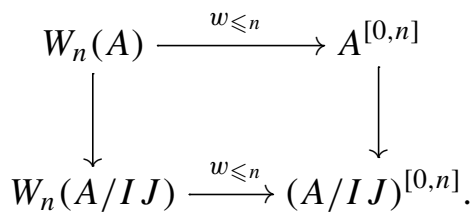

We want to show that the image of $x y$ in $W_{n}(A / I J)$ is zero. Since $A / I J$ is flat (even free) over $R$, the lower map $w_{\leqslant n}$ is injective, and so it is enough to show the image of $x y$ in $(A / I J)^{[0, n]}$ is zero. But by the naturality of the ghost map, we have $w_{\leqslant n}(x) \in I^{[0, n]}$ and $w_{\leqslant n}(y) \in J^{[0, n]}$. Therefore $w_{\leqslant n}(x y)$ lies in $(I J)^{[0, n]}$, which maps to zero in $(A / I J)^{[0, n]}$.

6.3. Remark. Although the proof of 6.2 given above uses some properties specific to Witt vector functors, the result is true for any representable ring-valued functor. See [Borger and Wieland 2005, 5.5].

6.4. Corollary. If $I$ is an ideal in an R-algebra $A$ and $I^{m}=0$, then $W_{R, E, n}(I)^{m}=0$.

6.5. Proposition. Let $\varphi: A \rightarrow B$ be a map of $R$-algebras. If it is surjective, then so is the map $W_{R, E, n}(\varphi): W_{R, E, n}(A) \rightarrow W_{R, E, n}(B)$.

Proof. By 5.4, we may assume $E$ consists of one ideal $\mathfrak{m}$. Since surjectivity can be checked Zariski locally on $R$, it is enough by 6.1 to assume $\mathfrak{m}$ is principal. Then using the Witt components, we can identify the set map underlying $W_{R, E, n}(\varphi)$ with the map $\varphi^{[0, n]}: A^{[0, n]} \rightarrow B^{[0, n]}$, which is clearly surjective.

6.6. Corollary. If $\varphi: A \rightarrow B$ is surjective, then

$$
W_{R, E, n}\left(A \times{ }_{B} A\right) \stackrel{W_{n}\left(\mathrm{pr}_{1}\right)}{\underset{W_{n}\left(\mathrm{pr}_{2}\right)}{\longrightarrow}} W_{R, E, n}(A) \stackrel{W_{n}(\varphi)}{\longrightarrow} W_{R, E, n}(B)
$$

is a coequalizer diagram. 
Proof. The functor $W_{n}$ is representable, and hence commutes with limits. (See 2.4.) Therefore $W_{n}\left(A \times{ }_{B} A\right)$ agrees with $W_{n}(A) \times_{W_{n}(B)} W_{n}(A)$, which is an equivalence relation on $W_{n}(A)$, the quotient by which is the image of $W_{n}(\varphi)$. By 6.5 , this is all of $W_{n}(B)$.

6.7. Remark. This result is particularly appealing when $A$ is $E$-flat and $B$ is not. Then we can describe $W_{n}(B)$ in terms of $W_{n}(A)$ and $W_{n}\left(A \times_{B} A\right)$, which are directly accessible because $A$ and $A \times{ }_{B} A$ are $E$-flat.

6.8. Proposition. Suppose E consists of one ideal $\mathfrak{m}$, and let $A$ be an $R$-algebra. For any $i \geqslant 0$, the map $\operatorname{Spec}\left(\mathrm{id} \otimes \bar{w}_{i}\right)$ of schemes induced by the ring map

$$
\mathrm{id} \otimes \bar{w}_{i}: R / \mathfrak{m} \otimes_{R} W_{R, E, n}(A) \longrightarrow R / \mathfrak{m} \otimes_{R} A / \mathfrak{m}^{n+1}
$$

is a universal homeomorphism. For $i=0$, it is a closed immersion defined by a square-zero ideal.

Proof. Write $W_{n}=W_{R, E, n}$ and so on. Consider the diagram

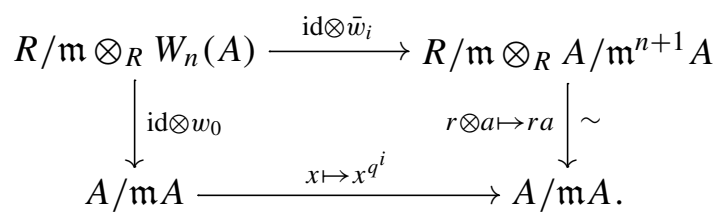

To show it commutes, it is enough to assume $\mathfrak{m}$ is principal, generated by $\pi$. Then commutativity follows from the obvious congruence

$$
w_{i}(a)=a_{0}^{q^{i}}+\pi a_{1}^{q^{i-1}}+\cdots+\pi^{i} a_{i} \equiv a_{0}^{q^{i}} \bmod \mathfrak{m} A,
$$

for any element $a=\left(a_{0}, a_{1}, \ldots\right)_{\pi} \in W(A)$.

Therefore, id $\otimes \bar{w}_{i}$ is the composition of a map whose kernel is a nil ideal and a power of the Frobenius map. The scheme maps induced by both of these are universal homeomorphisms.

Now let us show that id $\otimes w_{0}$ (which equals id $\otimes \bar{w}_{0}$ ) is a surjection with squarezero kernel. The map id $\otimes w_{0}$ is surjective by 1.21 (or 4.4 ). So let us show the square of its kernel is zero. By 4.4, the kernel of the map $W_{n}(A) \rightarrow R / \mathfrak{m} \otimes_{R} A$ is the ideal $V^{1} W_{n}(A)+\mathfrak{m} W_{n}(A)$. Hence it is enough to show $\left(V^{1} W_{n}(A)\right)^{2} \subseteq \mathfrak{m} W_{n}(A)$. This follows from (4-2-6).

6.9. Proposition. Let $\left(B_{i}\right)_{i \in I}$ be a family of A-algebras such that the induced map $\bigsqcup_{i} \operatorname{Spec} B_{i} \rightarrow \operatorname{Spec} A$ is surjective. Then the induced map

$$
\coprod_{i} \operatorname{Spec} W_{R, E, n}\left(B_{i}\right) \rightarrow \operatorname{Spec} W_{R, E, n}(A)
$$

is surjective. 
Proof. By 5.4, it is enough to assume $E$ consists of one ideal $\mathfrak{m}$. Further, it is enough to show surjectivity after base change to $R[1 / \mathfrak{m}]$ and to $R / \mathfrak{m}$. For $R[1 / \mathfrak{m}]$, it follows from 6.1 and the equality $W_{n}(C)=C^{[0, n]}$, when $\mathfrak{m}$ is the unit ideal. Now consider base change to $R / \mathfrak{m}$. By 6.8 , the ring $W_{n}(A) / \mathfrak{m} W_{n}(A)$ is a nilpotent extension of $A / \mathfrak{m} A$, and likewise for each $B_{i}$, and so we are reduced to showing that

$$
\bigsqcup_{i} \operatorname{Spec} B_{i} / \mathfrak{m} B_{i} \rightarrow \operatorname{Spec} A / \mathfrak{m} A
$$

is surjective. This is true since base change distributes over disjoint unions and preserves surjectivity.

6.10. Proposition. The R-algebra $\Lambda_{R, E, n}$ is finitely presented, and the functor $W_{R, E, n}$ preserves filtered colimits of $R$-algebras.

Proof. Since $W_{R, E, n}$ is represented by $\Lambda_{R, E, n}$, the two statements to be proved are equivalent. By 5.4, we may assume $E$ consists of a single ideal $\mathfrak{m}$. By [EGA 6, 2.7.1], the first statement can be verified fpqc locally on $R$, and in particular after base change to $R[1 / \mathfrak{m}]$ and to $R_{\mathfrak{m}}$. Therefore by (2-6-1), we can assume $\mathfrak{m}$ is generated by a single element $\pi$. But by 3.3 , the $R$-algebra $\Lambda_{R, E, n}$ is generated by the finite set $\theta_{\pi, 0}, \ldots, \theta_{\pi, n}$.

\section{Some general descent}

The purpose of this section is to record some facts about descent of étale algebras which we will use to prove our main result, Theorem 9.2. The results mention nothing about Witt vectors or anything else in this paper. So it would be reasonable to skip this section and refer back to it only as needed.

More precisely, we do the following. First, we set up some language and notation for descent, essentially repeating parts of Grothendieck's TDTE I [1966]. (It could not be otherwise.) Second, we prove an abstract result (7.10) relating gluing data and descent data for certain simple gluing constructions. Third, we recall Grothendieck's theorem (7.11) on integral descent of étale maps. Finally, we prove 7.12, which provides the plan of the proof of 9.2. Aside from the language of descent, only these three results will be used outside this section.

\section{Language}

7.1. Fibered categories. Let $C$ be a category with fibered products. Let $E$ be a category fibered over C. (See [Grothendieck 1966, A.1.1] or [SGA 1, VI.6.1].) For any object $S$ of $\mathrm{C}$, let $\mathrm{E}_{S}$ denote the fiber of $E$ over $S$. Let us say that a map $q: T \rightarrow S$ in $\mathrm{C}$ is an E-equivalence if $q^{*}: \mathrm{E}_{S} \rightarrow \mathrm{E}_{T}$ is an equivalence of categories, and let us say that $q$ is a universal E-equivalence if for any map $S^{\prime} \rightarrow S$ in C, the base change $q^{\prime}: S^{\prime} \times{ }_{S} T \rightarrow S^{\prime}$ is an E-equivalence. 
For the applications in the next section, the reader can take

$\mathrm{C}=$ the category of affine schemes,

$\mathrm{E}=$ the fibered category over $\mathrm{C}$ where $\mathrm{E}_{S}$ is the category of affine étale $S$-schemes and the functors $q^{*}$ are given by base change.

Then any closed immersion defined by a nil ideal is a universal E-equivalence [EGA 8, 18.1.2].

7.2. Composition notation. Let $S$ be an object of $\mathrm{C}$, and let $\mathrm{C}_{S \times S}$ denote the category of objects over $S \times S$. That is, an object of $C_{S \times S}$ is a pair $\left(T, \pi_{T}\right)$, where $T$ is an object of $C$ and $\pi_{T}$ is a map $T \rightarrow S \times S$, called its structure map; a morphism is a morphism in C commuting with the maps to $S \times S$. For such an object, let $\pi_{T, 1}, \pi_{T, 2}$ denote the composition of the structure map $T \rightarrow S \times S$ with the projections $\mathrm{pr}_{1}, \mathrm{pr}_{2}: S \times S \rightarrow S$. ( $\pi_{T, 1}$ is the "source" and $\pi_{T, 2}$ is the "target".) We will often abusively leave $\pi_{T}$ implicit and say that $T$ is an object of C.

Let $1_{S}$ denote the object $(S, \Delta)$ of $C_{S \times S}$, where $\Delta: S \rightarrow S \times S$ is the diagonal map.

Given two objects $T, U \in \mathrm{C}_{S \times S}$, define $T U \in \mathrm{C}_{S \times S}$ as follows. As an object of $C$, it is the fibered product

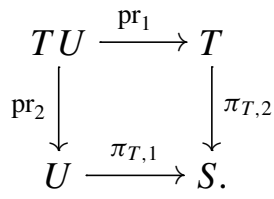

We give $T U$ the structure of an object of $C_{S \times S}$ with the map

$$
T U=T \times{ }_{S} U \stackrel{\left(\pi_{T, 1} \mathrm{opr}_{1}, \pi_{U, 2} \circ \mathrm{pr}_{2}\right)}{\longrightarrow} S \times S .
$$

7.3. Category objects and equivalence relations. A category object over $S$ is an object $R \in \mathrm{C}_{S \times S}$ together with maps

$$
\begin{aligned}
& e_{R}: 1_{S} \rightarrow R, \\
& c_{R}: R R \rightarrow R
\end{aligned}
$$

in $C_{S \times S}$ (called identity and composition) satisfying the usual identity and associativity axioms in the definition of a category. A morphism $f: R \rightarrow R^{\prime}$ of such category objects is defined to be a morphism in $C_{S \times S}$ satisfying the functor axioms, that is, such that

$$
f \circ e_{R}=e_{R} \circ f \quad \text { and } \quad c_{R^{\prime}} \circ f f=f \circ c_{R},
$$

where $f f$ denotes the map $R R \rightarrow R^{\prime} R^{\prime}$ induced by $f$. 
A category-object structure on a subobject $R \subseteq S \times S$ is a property of $R$ in that when it exists, it is unique. One might say that $R$ is a reflexive transitive relation on $S$. We say $R$ is an equivalence relation on $S$ if, in addition, the endomorphism $\left(\mathrm{pr}_{2}, \mathrm{pr}_{1}\right)$ of $S \times S$ that switches the two factors restricts to a map

$$
s: R \rightarrow R
$$

(which is of course unique when it exists).

7.4. Pre-actions (gluing data). Let $T$ be an object of $C_{S \times S}$. A pre-action of $T$ on an object $X \in \mathrm{E}_{S}$ is defined to be an isomorphism

$$
\varphi: \pi_{T, 2}^{*}(X) \stackrel{\sim}{\longrightarrow} \pi_{T, 1}^{*}(X)
$$

in $\mathrm{E}_{T}$. A pre-action is also called a gluing datum on $X$ relative to the pair of maps $\left(\pi_{T, 1}, \pi_{T, 2}\right)$. (Actually, Grothendieck [1966, A.1.4] calls $\varphi^{-1}$ the gluing datum.) Let

$$
\operatorname{PreAct}(T, X)
$$

denote the set of pre-actions of $T$ on $X$. Any map $T \rightarrow T^{\prime}$ in $C_{S \times S}$ naturally induces a map

$$
\operatorname{PreAct}\left(T^{\prime}, X\right) \rightarrow \operatorname{PreAct}(T, X) .
$$

If $f: X \rightarrow X^{\prime}$ is a morphism in $\mathrm{E}_{S}$ between objects $X, X^{\prime}$ with pre-actions $\varphi, \varphi^{\prime}$, then we say $f$ is $T$-equivariant if the diagram

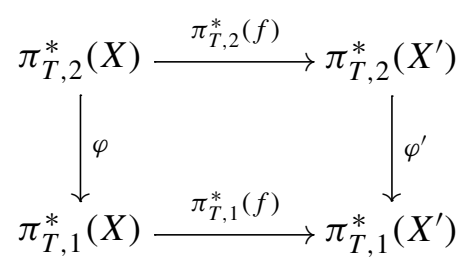

commutes.

In this way, the objects of $\mathrm{E}_{S}$ equipped with a pre-action of $T$ form a category.

7.5. Actions. Now let $R$ be a category object over $S$. An action of $R$ on $X$ is defined to be a pre-action $\varphi$ of $R$ on $X$ such that the diagram

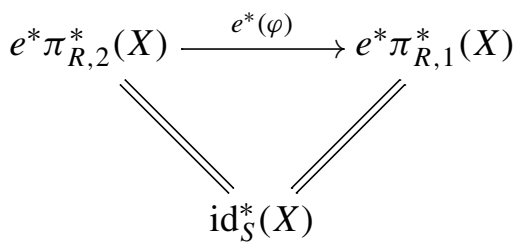


and the diagram

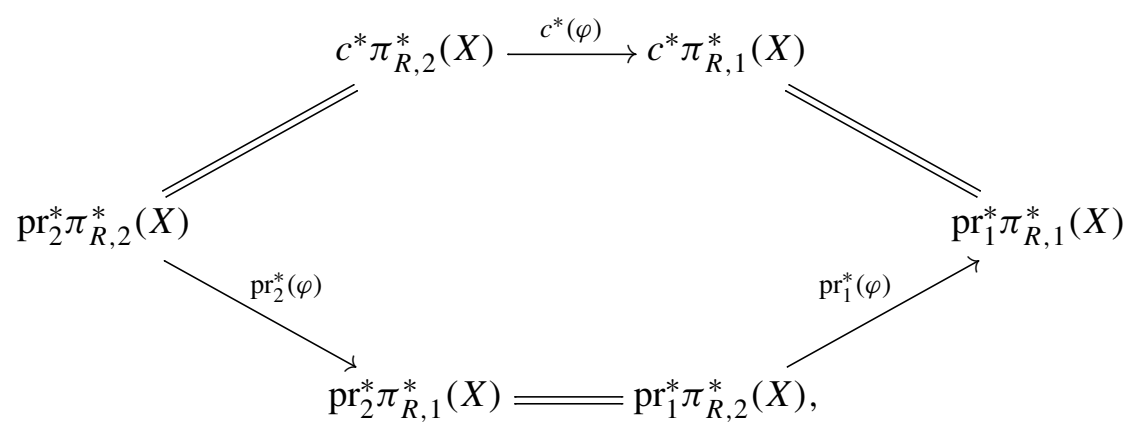

commute. Here, $\mathrm{pr}_{1}$ and $\mathrm{pr}_{2}$ denote the projections $R R \rightarrow R$ onto the first and second factors, and the morphisms represented by equality signs are the isomorphisms induced by the canonical structure maps $(g \circ f)^{*} \stackrel{\sim}{\rightarrow} f^{*} \circ g^{*}$ (denoted by $c_{f, g}$ in [Grothendieck 1966, A.1.1(ii)]) of the fibered category E corresponding to the equalities

$$
\pi_{R, 2} \circ e=\mathrm{id}_{S}=\pi_{R, 1} \circ e
$$

and

$$
\begin{aligned}
\pi_{R, 2} \circ c & =\pi_{R, 2} \circ \mathrm{pr}_{2}, \\
\pi_{R, 1} \circ \mathrm{pr}_{2} & =\pi_{R, 2} \circ \mathrm{pr}_{1}, \\
\pi_{R, 1} \circ c & =\pi_{R, 1} \circ \mathrm{pr}_{1} .
\end{aligned}
$$

We will often use the following more succinct, if slightly abusive, expressions of the commutativity of the diagrams above:

$$
e^{*}(\varphi)=\operatorname{id}_{X}, \quad c^{*}(\varphi)=\left(\operatorname{pr}_{1}^{*} \varphi\right) \circ\left(\operatorname{pr}_{2}^{*} \varphi\right) .
$$

Let $\operatorname{Act}(R, X)$ denote the set of actions of $R$ on $X$. A morphism $R \rightarrow R^{\prime}$ of category objects induces a map

$$
\operatorname{Act}\left(R^{\prime}, X\right) \longrightarrow \operatorname{Act}(R, X)
$$

in the obvious way.

Last, note that if $R$ is an equivalence relation, the diagram

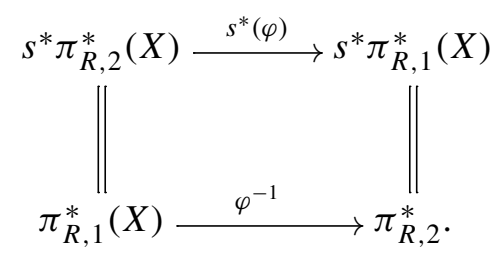

commutes. This follows immediately from (7-5-1). The abbreviated version is

$$
s^{*}(\varphi)=\varphi^{-1} \text {. }
$$


7.6. Descent data. Let $q: S^{\prime} \rightarrow S$ be a map in C, and put

$$
R\left(S^{\prime} / S\right)=S^{\prime} \times{ }_{S} S^{\prime}
$$

View $R\left(S^{\prime} / S\right)$ as an object in $C_{S^{\prime} \times S^{\prime}}$ by taking $\pi_{R\left(S^{\prime} / S\right)}$ to be the evident monomorphism

$$
R\left(S^{\prime} / S\right)=S^{\prime} \times{ }_{S} S^{\prime} \longrightarrow S^{\prime} \times S^{\prime}
$$

Then $R\left(S^{\prime} / S\right)$ is an equivalence relation on $S^{\prime}$. An action $\varphi$ of $R\left(S^{\prime} / S\right)$ on an object $X^{\prime}$ of $E_{S^{\prime}}$ is also called a descent datum on $X^{\prime}$ from $S^{\prime}$ to $S$. (Again, it is actually $\varphi^{-1}$ that is called the descent datum in [Grothendieck 1966].) We might call $R\left(S^{\prime} / S\right)$ the descent, or Galois, groupoid of the map $q: S^{\prime} \rightarrow S$.

Because the two compositions $R\left(S^{\prime} / S\right)=S^{\prime} \times{ }_{S} S^{\prime} \rightrightarrows S^{\prime} \rightarrow S$ are equal, for any object $X \in \mathrm{E}_{S}$, the object $q^{*}(X)$ of $\mathrm{E}_{S^{\prime}}$ has a canonical pre-action of $R\left(S^{\prime} / S\right)$, and it is easy to check that this is an action. We say that $q$ is a descent map for the fibered category $\mathrm{E}$ if the functor from $\mathrm{E}_{S}$ to the category of objects of $\mathrm{E}_{S^{\prime}}$ with an $R$-action is fully faithful. We say it is an effective descent map if it is an equivalence.

7.7. When gluing data is descent data. Now suppose we have a diagram

$$
S^{\prime \prime} \longrightarrow S^{\prime} \longrightarrow S
$$

in C such that the two compositions $S^{\prime \prime} \rightrightarrows S$ are equal. The universal property of products gives a map

$$
S^{\prime \prime} \longrightarrow S^{\prime} \times{ }_{S} S^{\prime}=R\left(S^{\prime} / S\right) .
$$

For any object $X^{\prime} \in \mathrm{E}_{S^{\prime}}$, this map induces a function

$$
\operatorname{Act}\left(R\left(S^{\prime} / S\right), X^{\prime}\right) \longrightarrow \operatorname{PreAct}\left(S^{\prime \prime}, X^{\prime}\right) \text {. }
$$

Let us say that gluing data on $X^{\prime}$ is descent data relative to the diagram (7-7-1) when this map is a bijection.

\section{Gluing two objects}

Here we spell out in (perhaps excessive) detail some basic facts about equivalence relations on disjoint unions that are E-trivial, but not necessarily trivial, on each factor.

From now on, let $\mathrm{C}$ denote the category of affine schemes, schemes, or algebraic spaces. (We only need some weak hypotheses on coproducts in C, but let us not bother to determine which ones we need.)

7.8. Equivalence relations on a disjoint union. Suppose $S$ is a coproduct $S_{a}+S_{b}$ of two objects $S_{a}, S_{b} \in \mathrm{C}$. (We use the symbols $a, b$ to index the summands only to emphasize their distinction from the symbols 1,2 that index the factors in the 
product $S \times S$.) Let $R$ be an equivalence relation on $S$, and let $R_{i j}$ denote $R \times S \times S$ $\left(S_{i} \times S_{j}\right)$, for any $i, j \in\{a, b\}$. Let $\pi_{R_{i j}, 1}$ denote the evident composition

$$
R_{i j}=R \times_{S \times S}\left(S_{i} \times S_{j}\right) \stackrel{\mathrm{pr}_{1}}{\longrightarrow} S_{i}
$$

and $\pi_{R_{i j}, 2}$ the analogous map $R_{i j} \rightarrow S_{j}$. We will sometimes view $R_{i j}$ as an object of $C_{S \times S}$ using the induced map $R_{i j} \rightarrow S_{i} \times S_{j} \rightarrow S \times S$.

Let $e_{i}: S_{i} \rightarrow R_{i i}$ and $c_{i j k}: R_{i j} R_{j k} \rightarrow R_{i k}$ and $s_{i j}: R_{i j} \rightarrow R_{j i}$ denote the evident restrictions of $e$ and $c$ and $s$.

7.9. Actions over a disjoint union. For any object $X$ over $S$, write $X_{a}=S_{a} \times_{S} X$ and $X_{b}=S_{b} \times{ }_{S} X$.

For any pre-action

$$
\varphi: \pi_{R, 2}^{*} X \longrightarrow \pi_{R, 1}^{*} X,
$$

of $R$ on $X$, let us write $\varphi_{i j}$ for the restriction of $\varphi$ to $R_{i j}$. In order for this pre-action to be an action, it is necessary and sufficient that for all $i, j, k \in\{a, b\}$ we have

$$
\begin{aligned}
e_{i}^{*}\left(\varphi_{i i}\right) & =\mathrm{id}_{X_{i}}, \\
c_{i j k}^{*}\left(\varphi_{i k}\right) & =\operatorname{pr}_{1}^{*}\left(\varphi_{i j}\right) \circ \operatorname{pr}_{2}^{*}\left(\varphi_{j k}\right) .
\end{aligned}
$$

This is just a restatement of (7-5-1), summand by summand. In that case, (7-5-2) becomes

$$
s_{i j}^{*}\left(\varphi_{j i}\right)=\varphi_{i j}^{-1} .
$$

7.10. Proposition. Let $R$ be an equivalence relation on $S=S_{a}+S_{b}$ such that for $i=a, b$, the map $e_{i}: S_{i} \rightarrow R_{i i}$ is a universal E-equivalence. Then for any object $X \in \mathrm{E}_{S}$, the map

$$
\operatorname{Act}(R, X) \stackrel{\varphi \mapsto \varphi_{b a}}{\longrightarrow} \operatorname{PreAct}\left(R_{b a}, X\right)
$$

is a bijection.

Proof. Let us first show injectivity. Let $\varphi$ and $\varphi^{\prime}$ be actions of $R$ on $X$ such that $\varphi_{b a}=\varphi_{b a}^{\prime}$. We need to show that this implies $\varphi_{i j}=\varphi_{i j}^{\prime}$ for all $i, j \in\{a, b\}$. Consider each case separately. For $i j=b a$, it is true by assumption. When $i j=a b,(7-9-4)$ and the given equality $\varphi_{b a}=\varphi_{b a}^{\prime}$ imply

$$
\varphi_{a b}=s_{b a}^{*}\left(\varphi_{a b}\right)^{-1}=s_{b a}^{*}\left(\varphi_{a b}^{\prime}\right)^{-1}=\varphi_{a b}^{\prime} .
$$

When $i=j$, since $e_{i}$ is an E-equivalence, it is enough to show $e_{i}^{*}\left(\varphi_{i i}\right)=e_{i}^{*}\left(\varphi_{i i}^{\prime}\right)$. But by (7-9-2), we have

$$
e_{i}^{*}\left(\varphi_{i i}\right)=\operatorname{id}_{X_{i}}=e_{i}^{*}\left(\varphi_{i i}^{\prime}\right) .
$$

Therefore $\varphi=\varphi^{\prime}$, which proves injectivity. 
Now consider surjectivity. Let $\varphi_{b a}$ be a pre-action of $R_{b a}$ on $X$. Define

$$
\varphi_{a b}=s_{a b}^{*}\left(\varphi_{b a}\right)^{-1}
$$

and for $i=a, b$ define $\varphi_{i i}$ to be the map such that

$$
e_{i}^{*}\left(\varphi_{i i}\right)=\mathrm{id}_{X_{i}},
$$

which exists and is unique because $e_{i}$ is an E-equivalence. We need to check that the pre-action $\varphi=\varphi_{a a}+\varphi_{a b}+\varphi_{b a}+\varphi_{b b}$ of $R$ on $X$ is actually an action. To do this, we will verify the relations (7-9-2) and (7-9-3).

The identity axiom (7-9-2) holds because it is the defining property (7-10-2) of $\varphi_{i i}$.

Now consider the associativity axiom (7-9-3) for the various possibilities for $i j k$. Since $i, j, k \in\{a, b\}$, two of $i, j, k$ must be equal.

If $i=j$, the composition $f$

$$
R_{j k} \stackrel{\mathrm{pr}_{2}^{-1}}{\sim} S_{j j} R_{j k} \stackrel{e_{j} \times \mathrm{id}}{\longrightarrow} R_{j j} R_{j k}
$$

is an E-equivalence, because it is a base change of the universal E-equivalence $e_{j}$. Therefore it is enough to show

$$
f^{*} c_{j j k}^{*}\left(\varphi_{j k}\right)=f^{*} \operatorname{pr}_{1}^{*}\left(\varphi_{j j}\right) \circ f^{*} \operatorname{pr}_{2}^{*}\left(\varphi_{j k}\right) .
$$

By the equality $\operatorname{pr}_{1} \circ f=e_{j} \circ \pi_{R_{j k}, 1}$ and (7-10-2), we have

$$
f^{*} \operatorname{pr}_{1}^{*}\left(\varphi_{j j}\right)=\pi_{R_{j k}, 1}^{*} e_{j}^{*}\left(\varphi_{j j}\right)=\pi_{R_{j k}, 1}^{*}\left(\mathrm{id}_{X_{j}}\right)=\mathrm{id} .
$$

On the other hand, by $c_{j j k} \circ f=\mathrm{id}_{R_{j k}}=\operatorname{pr}_{2} \circ f$, we have $f^{*} c_{j j k}^{*}\left(\varphi_{j k}\right)=f^{*} \operatorname{pr}_{2}^{*}\left(\varphi_{j k}\right)$. Equation (7-10-3) then follows.

The case $j=k$ is similar to the case $i=j$. (Or apply $s$ to the case $i=j$.)

Last, suppose $i=k$. The following diagram is easily checked to be cartesian:

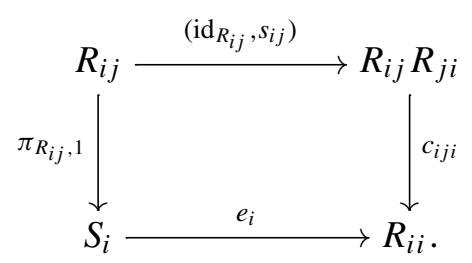

(This is just another expression of the existence and uniqueness of inverses in a groupoid.) Since $e_{i}$ is a universal E-equivalence, $\left(\mathrm{id}_{R_{i j}}, s_{i j}\right)$ is an E-equivalence. So it is enough to show axiom (7-9-3) after applying $\left(\mathrm{id}_{R_{i j}}, s_{i j}\right)^{*}$, that is, to show

$$
\left(\operatorname{id}_{R_{i j}}, s_{i j}\right)^{*} c_{i j i}^{*}\left(\varphi_{i i}\right)=\left(\operatorname{id}_{R_{i j}}, s_{i j}\right)^{*} \operatorname{pr}_{1}^{*}\left(\varphi_{i j}\right) \circ\left(\operatorname{id}_{R_{i j}}, s_{i j}\right)^{*} \operatorname{pr}_{2}^{*}\left(\varphi_{j i}\right) .
$$


By the commutativity of (7-10-4) and (7-10-2), we have

$$
\left(\operatorname{id}_{R_{i j}}, s_{i j}\right)^{*} c_{i j i}^{*}\left(\varphi_{i i}\right)=\pi_{R_{i j}, 1}^{*} e_{i}^{*}\left(\varphi_{i i}\right)=\pi_{R_{i j}, 1}^{*}\left(\operatorname{id}_{X_{i}}\right)=\mathrm{id} .
$$

Combining this with the equation $\varphi_{j i}=s_{j i}^{*}\left(\varphi_{i j}\right)^{-1},(7-10-5)$ reduces to

$$
\left(\operatorname{id}_{R_{i j}}, s_{i j}\right)^{*} \operatorname{pr}_{1}^{*}\left(\varphi_{i j}\right)=\left(\operatorname{id}_{R_{i j}}, s_{i j}\right)^{*} \operatorname{pr}_{2}^{*} s_{j i}^{*}\left(\varphi_{i j}\right) .
$$

But this holds because we have

$$
\operatorname{pr}_{1} \circ\left(\operatorname{id}_{R_{i j}}, s_{i j}\right)=\mathrm{id}_{R_{i j}}=s_{j i} \circ s_{i j}=s_{j i} \circ \mathrm{pr}_{2} \circ\left(\operatorname{id}_{R_{i j}}, s_{i j}\right) .
$$

Therefore the equations in (7-9-3) hold for all $i, j, k$, and so the pre-action is an action.

\section{Grothendieck's theorem}

Recall that a map Spec $B \rightarrow \operatorname{Spec} A$ of affine schemes is said to be integral if the corresponding ring map $A \rightarrow B$ is integral (and not necessarily injective).

7.11. Theorem. Every surjective integral map $Y \rightarrow X$ of affine schemes is an effective descent map for the fibered category $\mathrm{E}$ over $\mathrm{C}$ of (7-1-1).

This theorem is proven in [SGA 1, IX 4.7] up to two details. First, the argument given there covers only morphisms $Y \rightarrow X$ which are finite and of finite presentation; and second, the statement there has no affineness in the assumptions or in the conclusion. The first point can be handled by a standard limiting argument (or one can apply [Rydh 2010, Theorem 5.17 plus Remark 2.5(1b)]). The second point can be handled with Chevalley's theorem; the form most convenient here would the final one [Rydh 2009, Theorem 8.1], which is free of noetherianness, separatedness, finiteness, and scheme-theoretic assumptions.

\section{Gluing and descent of étale algebras}

7.12. Proposition. Consider a diagram of rings

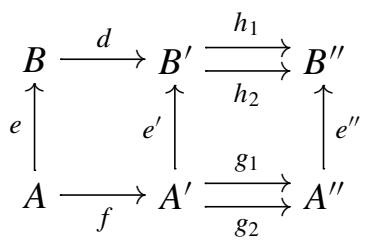

such that $h_{i} \circ e^{\prime}=e^{\prime \prime} \circ g_{i}$, for $i=1,2$. Also assume that

(a) the two parallel right-hand squares are cocartesian,

(b) both rows are equalizer diagrams,

(c) relative to the lower row, gluing data on any étale $A^{\prime}$-algebra is descent data, 
(d) $f$ satisfies effective descent for the fibered category of étale algebras, and

(e) $e^{\prime}$ is étale.

Then e is étale and the left-hand square is cocartesian.

Note that when we use the language of descent in the category of rings (as in (c) and (d)), we understand that it refers to the corresponding statements in the opposite category.

Proof. Property (a) equips the étale $A^{\prime}$-algebra $B^{\prime}$ with gluing data $\varphi$ relative to $\left(g_{1}, g_{2}\right)$. Indeed, take $\varphi$ to be the composition

$$
A^{\prime \prime} \otimes_{g_{1}, A^{\prime}} B^{\prime} \stackrel{\sim}{\longrightarrow} B^{\prime \prime} \stackrel{\sim}{\longrightarrow} A^{\prime \prime} \otimes_{g_{2}, A^{\prime}} B^{\prime} .
$$

By property (c), this gluing data comes from unique descent data relative to $f$. Therefore by (d) and (e), the $A^{\prime}$-algebra $B^{\prime}$ descends to an étale $A$-algebra $C$.

Now apply the functor $C \otimes_{A}-$ to the lower row of diagram (7-12-1). By (a) and the definition of descent, the result can be identified with the sequence

$$
C \longrightarrow B^{\prime} \underset{h_{2}}{\stackrel{h_{1}}{\longrightarrow}} B^{\prime \prime} .
$$

This sequence is also an equalizer diagram, because the lower row of (7-12-1) is an equalizer diagram, by (b), and because $C$ is étale over $A$ and hence flat. Again by (b), the upper row of (7-12-1) is an equalizer diagram, and so we have $C=B$. Therefore, $B$ is an étale $A$-algebra and the left-hand square is cocartesian.

\section{Ghost descent in the single-prime case}

We return to the notation of 1.2. Suppose $E$ consists of a single maximal ideal $\mathfrak{m}$, and fix an integer $n \geqslant 1$. Write $W_{n}=W_{R, \mathfrak{m}, n}$, and so on. Let $A$ be an $R$-algebra, and let $\alpha_{n}$ denote the map

$$
W_{n}(A) \stackrel{\alpha_{n}}{\longrightarrow} W_{n-1}(A) \times A
$$

given by the canonical projection on the factor $W_{n-1}(A)$ and the $n$-th ghost component $w_{n}$ on the factor $A$. Let $I_{n}(A)$ denote the kernel of $\alpha_{n}$. For example, if $\mathfrak{m}$ is generated by $\pi$, then in terms of the Witt components, we have

$$
I_{n}(A)=\left\{(0, \ldots, 0, a)_{\pi} \in A^{[0, n]} \mid \pi^{n} a=0\right\} .
$$

8.1. Proposition. (a) $\alpha_{n}$ is an integral ring homomorphism.

(b) The kernel $I_{n}(A)$ of $\alpha_{n}$ is a square-zero ideal.

(c) If $A$ is $\mathfrak{m}$-flat, then $\alpha_{n}$ is injective. 
(d) The diagram

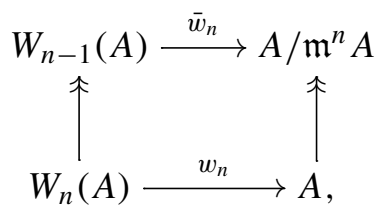

where the vertical maps are the canonical ones, is cocartesian.

(e) View A as a $W_{n}(A)$-algebra by the map $w_{n}: W_{n}(A) \rightarrow A$. Then every element in the kernel of the multiplication map $A \otimes_{W_{n}(A)} A \longrightarrow A$ is nilpotent.

(f) In the diagram

$$
W_{n}(A) \stackrel{\alpha_{n}}{\longrightarrow} W_{n-1}(A) \times A \underset{\overline{\mathrm{pr}}_{2}}{\stackrel{\bar{w}_{n} \circ \mathrm{pr}_{1}}{\longrightarrow}} A / \mathfrak{m}^{n} A
$$

where $\overline{\mathrm{pr}}_{2}$ denotes the reduction of $\mathrm{pr}_{2}$ modulo $\mathrm{m}^{n}$, the image of $\alpha_{n}$ agrees with the equalizer of $\bar{w}_{n} \circ \mathrm{pr}_{1}$ and $\overline{\mathrm{pr}}_{2}$.

Proof. (a): It is enough to show that each factor of $W_{n-1}(A) \times A$ is integral over $W_{n}(A)$. The first factor is a quotient ring, and hence integral. Now consider an element $a \in A$. Then $a^{q^{n}}$ is the image in $A$ of the Teichmüller lift $[a] \in W_{n}(A)$. (See 1.21.) Therefore the second factor is also integral over $W_{n}(A)$.

(b) It suffices to show this after base change to $R[1 / \mathfrak{m}] \times R_{\mathfrak{m}}$. Therefore, by 6.1 , we may assume $\mathfrak{m}$ is generated by a single element $\pi$. Then an element of the kernel of $\alpha_{n}$ will be of the form $V_{\pi}^{n}[a]=(0, \ldots, 0, a)_{\pi}$, where $\pi^{n} a=0$. On the other hand, by (4-2-6) we have

$$
\left(V_{\pi}^{n}[a]\right)\left(V_{\pi}^{n}[b]\right)=\pi^{n} V_{\pi}^{n}[a b]=\left(0, \ldots, 0, \pi^{n} a b\right)_{\pi}=0 .
$$

(c) We have $\left(w_{\leqslant n-1} \times \operatorname{id}_{A}\right) \circ \alpha_{n}=w_{\leqslant n}$. Since $A$ is $\mathfrak{m}$-flat, the map $w_{\leqslant n}$ is injective 2.7, and hence so is $\alpha_{n}$.

(d) As above, it is enough by 6.1 to assume $\mathfrak{m}$ is generated by a single element $\pi$. Then we have

$$
A \otimes_{W_{n}(A)} W_{n-1}(A)=A \otimes_{W_{n}(A)} W_{n}(A) / V^{n} W_{n}(A)=A / w_{n}\left(V^{n} W_{n}(A)\right) A .
$$

Examining the Witt polynomials (3-1-1) shows $w_{n}\left(V^{n} W_{n}(A)\right)=\pi^{n} A$.

(e) Again, by 6.1 we may assume $\mathfrak{m}$ is generated by a single element $\pi$. To show every element $x \in I$ is nilpotent, it is enough to restrict $x$ to a set of generators. Therefore it is enough to show $(1 \otimes a-a \otimes 1)^{q^{n}}=0$ for every element $a \in A$.

Now suppose that, for $j=0, \ldots, q^{n}$, we could show

$$
\left(\begin{array}{c}
q^{n} \\
j
\end{array}\right) a^{j} \in \operatorname{im}\left(w_{n}\right) .
$$


Then we would have

$$
\begin{aligned}
(1 \otimes a-a \otimes 1)^{q^{n}} & =\sum_{j}(-1)^{j}\left(\begin{array}{c}
q^{n} \\
j
\end{array}\right) a^{j} \otimes a^{q^{n}-j}=\sum_{j}(-1)^{j} \otimes\left(\begin{array}{c}
q^{n} \\
j
\end{array}\right) a^{j} a^{q^{n}-j} \\
& =(1 \otimes a-1 \otimes a)^{q^{n}}=0,
\end{aligned}
$$

which would complete the proof. So let us show (8-1-2).

Let $f=\operatorname{ord}_{p}(q)$ and $i=\operatorname{ord}_{p}(j)$. Then we have

$$
\operatorname{ord}_{p}\left(\begin{array}{c}
q^{n} \\
j
\end{array}\right)=\operatorname{ord}_{p}\left(q^{n} j^{-1}\right)+\operatorname{ord}_{p}\left(\begin{array}{c}
q^{n}-1 \\
j-1
\end{array}\right) \geqslant n f-i .
$$

It follows that $\left(\begin{array}{c}q^{n} \\ j\end{array}\right) a^{j}$ is an $R$-linear multiple of $\pi^{n f-i} a^{j}$. Since $w_{n}$ is an $R$ algebra map, it is therefore enough to show

$$
\pi^{n f-i} a^{j} \in \operatorname{im}\left(w_{n}\right) .
$$

Now, for $b \in A$ and $s=0, \ldots, n$, we have $\pi^{n-s} b^{q^{s}}=w_{n}\left(V_{\pi}^{n-s}[b]\right)$, and therefore $\pi^{n-s} b^{q^{s}}$ is in the image of $w_{n}$. So to show (8-1-3), it is enough to find an integer $s$ and an element $b \in A$ such that $\pi^{n-s} b^{q^{s}}$ is an $R$-linear divisor of $\pi^{n f-i} a^{j}$. In particular, it is sufficient for $b$ and $s$ to satisfy $b^{q^{s}}=a^{j}$ and $n-s \leqslant n f-i$.

Take $s$ to be the greatest integer at most $i f^{-1}$. Then we have $q^{s} \mid j$; so if we set $b=a^{j / q^{s}} \in A$, we have $b^{q^{s}}=a^{j}$. It remains to show $n-s \leqslant n f-i$. This is equivalent to $n-i f^{-1} \leqslant n f-i$, which is in turn equivalent to $(1-f)\left(n-i f^{-1}\right) \leqslant 0$. And this holds because $1-f \leqslant 0$ and $n-i f^{-1} \geqslant 0$. (Recall that $j \leqslant q^{n}$.) This completes the proof of (e).

(f) As above, we may assume that $\mathfrak{m}$ can be generated by a single element $\pi$. For any element $a=\left(a_{0}, \ldots, a_{n}\right)_{\pi} \in W_{n}(A)$, we have

$$
\alpha_{n}(a)=\left(\left(a_{0}, \ldots, a_{n-1}\right), a_{0}^{q^{n}}+\cdots+\pi^{n-1} a_{n-1}^{q}+\pi^{n} a_{n}\right) .
$$

Therefore an element $\left(\left(a_{0}, \ldots, a_{n-1}\right), b\right) \in W_{n-1}(A) \times A$ lies in the image of $\alpha_{n}$ if and only if

$$
a_{0}^{q^{n}}+\cdots+\pi^{n-1} a_{n-1}^{q} \equiv b \quad \bmod \mathfrak{m}^{n} A,
$$

which is exactly what we needed to show.

8.2. Corollary. For any R-algebra A, the ghost map

$$
w_{\leqslant n}: W_{n}(A) \longrightarrow A^{[0, n]}
$$

is integral, and its kernel $J$ satisfies $J^{2^{n}}=0$.

Proof. By 8.1 and induction on $n$.

8.3. Theorem. (a) The map $\alpha_{n}$ is an effective descent map for the fibered category of étale algebras. 
(b) Relative to the diagram

$$
W_{n}(A) \stackrel{\alpha_{n}}{\longrightarrow} W_{n-1}(A) \times A \underset{\overline{\mathrm{pr}}_{2}}{\stackrel{\bar{w}_{n} \circ \mathrm{pr}_{1}}{\longrightarrow}} A / \mathfrak{m}^{n} A,
$$

gluing data on any étale $W_{n-1}(A) \times A$-algebra is descent data (7.7).

(c) If $A$ is $\mathfrak{m}$-flat, then for any $A^{\prime}$-algebra $B^{\prime}$ equipped with gluing data $\varphi$, the descended A-algebra is the subring $B$ of $B^{\prime}$ on which the following diagram commutes:

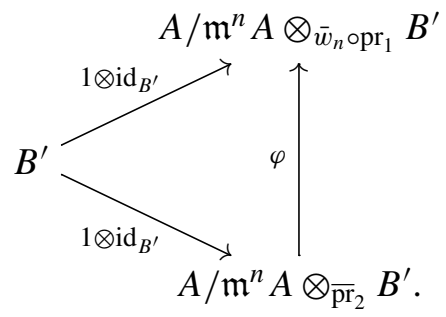

Proof. (a) This follows from Grothendieck's Theorem 7.11 and 8.1(a)-(b).

(b) We will use 7.10, where $C$ and $E$ are as in (7-1-1). In the notation of 7.8, put

$$
S_{a}=\operatorname{Spec} W_{n}(A) \quad \text { and } \quad S_{b}=\operatorname{Spec} A .
$$

Let $\Gamma$ be the equivalence relation $S \times{ }_{\operatorname{Spec}} W_{n}(A) S$ on $S$. By 8.1(d), we have $\Gamma_{b a}=$ $\operatorname{Spec} A / \mathfrak{m}^{n} A$. The map $e_{a}$ is an isomorphism because $W_{n-1}(A)$ is a quotient ring of $W_{n}(A)$. The map $e_{b}$ is a nil immersion, by 8.1(e), and hence is an E-equivalence. Thus we can apply 7.10, which says that a $\Gamma$-action is the same as a $\Gamma_{b a}$ pre-action. In other words, gluing data is descent data.

(c) This will follow from 7.12 once we verify the hypotheses. 7.12(a)-(b) are clear; 7.12(c) follows from (b) above; 7.12(d) follows from (a) above; and 7.12(e) follows from the definition of $B$, for the top row of (7-12-1), and from 8.1(c) and (f), for the bottom row.

8.4. Remark. For any ring $C$, let $\mathrm{EtAlg}_{C}$ denote the category of étale $C$-algebras. Then another way of expressing part (b) of this theorem is that the induced functor

$$
\mathrm{EtAlg}_{W_{n}(A)} \longrightarrow \mathrm{EtAlg}_{W_{n-1}(A)} \times \mathrm{EtAlg}_{A / \mathfrak{m}^{n} A} \mathrm{EtAlg}_{A}
$$

is an equivalence. (Of course, the fibered product of categories is taken in the weak sense.) In particular, we can prove things about étale $W_{n}(A)$-algebras by induction on $n$. This is the main technique in the proof of 9.2. But it also seems interesting in its own right and will probably have applications beyond the present paper. 
8.5. Remark. If we let $\bar{W}_{n}(A)$ denote the image of $\alpha_{n}$, the induced diagram

$$
\bar{W}_{n}(A) \longrightarrow W_{n-1}(A) \times A \underset{\overline{\mathrm{pr}}_{2}}{\stackrel{\bar{w}_{n} \circ \mathrm{pr}_{1}}{\longrightarrow}} A / \mathfrak{m}^{n} A .
$$

satisfies all the conclusions of the theorem above, regardless of whether $A$ is $\mathfrak{m}$-flat.

Indeed, it is an equalizer diagram by 8.1 (f) and the definition of $\bar{W}_{n}(A)$; it is an effective descent map by 8.3 and 8.1(b). Last, because $\bar{W}_{n}(A)$ is the image of $\alpha_{n}$, gluing (resp. descent) data relative to $\bar{W}_{n}(A)$ agrees with gluing (resp. descent) data relative to $W_{n}(A)$. In particular, gluing data relative to $\bar{W}_{n}(A)$ is descent data.

\section{9. $W$ and étale morphisms}

We return to the general context of 1.2. In particular, $E$ is no longer required to consist of one ideal.

9.1. Lemma. Consider a commutative square of affine schemes (or any schemes)

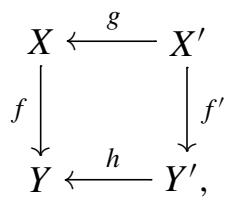

and let $U$ be an open subscheme of $Y$. Suppose that

(a) $f$ and $f^{\prime}$ are étale,

(b) the square above becomes cartesian after the base change $U \times_{Y}-$, and

(c) $g$ and $h$ become surjective and universally injective after the base change $(Y-U) \times_{Y}-$.

Then the square above is cartesian.

Proof. Let $e$ denote the induced map $\left(g, f^{\prime}\right): X^{\prime} \rightarrow X \times{ }_{Y} Y^{\prime}$. It is enough to show $e$ is étale, surjective, and universally injective [EGA 8, 17.9.1]. The composition of $e$ with $\mathrm{pr}_{2}: X \times_{Y} Y^{\prime} \rightarrow Y^{\prime}$ is $f^{\prime}$. Because $f$ is étale, so is its base change $\mathrm{pr}_{2}$. Combining this with the étaleness of $f^{\prime}$ implies that $e$ is étale [EGA 8, 17.3.4].

The surjectivity and universal injectivity of $e$ can be checked after base change over $Y$ to $U$ and to $Y-U$. By assumption $e$ becomes an isomorphism after base change to $U$. In particular, it becomes surjective and universally injective.

Let $\bar{e}, \bar{g}, \bar{h}$ denote the maps $e, g, h$ pulled back from $Y$ to $Y-U$. Let $\bar{h}^{\prime}$ denote the base change of $\bar{h}$ from $Y$ to $X$. Then, as above, we have $\bar{g}=\bar{h}^{\prime} \circ \bar{e}$. Since $\bar{h}$ is universally injective, so is $\bar{h}^{\prime}$. Combining this with the fact that $\bar{g}$ is universally injective, implies that $\bar{e}$ is as well [EGA 1, 3.5.6-7]. Finally $\bar{e}$ is surjective since $\bar{h}^{\prime}$ is injective and $\bar{g}$ is surjective. 
9.2. Theorem. For any étale map $\varphi: A \rightarrow B$ and any element $n \in \mathbb{N}^{(E)}$, the induced map $W_{R, E, n}(\varphi): W_{R, E, n}(A) \rightarrow W_{R, E, n}(B)$ is étale.

Proof. By 5.4, it is enough to assume $E$ consists of a single maximal ideal $\mathfrak{m}$. Also, it will simplify notation if we assume $\mathfrak{m}$ is principal, generated by an element $\pi$. We may do this by 6.1 and because it is enough to show étaleness after applying $R_{\mathfrak{m}} \otimes_{R}-$ and $R[1 / \mathfrak{m}] \otimes_{R}-$. Let us write $W_{n}=W_{R, E, n}$.

We will reason by induction on $n$, the case $n=0$ being clear because $W_{0}$ is the identity functor. So from now on, assume $n \geqslant 1$.

Let $\bar{W}_{n}(A)$ denote the image of $\alpha_{n}: W_{n}(A) \rightarrow W_{n-1}(A) \times A$, and let $\bar{\alpha}_{n}$ denote the induced injection $\bar{W}_{n}(A) \rightarrow W_{n-1}(A) \times A$. Define $\bar{W}_{n}(B)$ and $\bar{\alpha}_{n}$ for $B$ similarly.

Step 1: $\bar{W}_{n}(B)$ is étale over $\bar{W}_{n}(A)$. To show this, it suffices to verify conditions (a)-(e) of 7.12 for the diagram

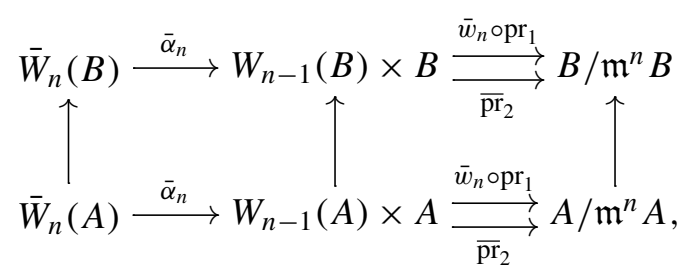

where the vertical maps are induced by $\varphi$ and functoriality. We know 7.12(a) holds by induction. Conditions 7.12(c)-(d) hold by 8.3 (or 8.5). Condition 7.12(e) was shown already in 8.1(f). Now consider 7.12(b). It is clear that the square of $\overline{\mathrm{pr}}_{2}$ maps is cocartesian. So, all that remains is to check that the square of $\bar{w}_{n} \circ \mathrm{pr}_{1}$ maps is cocartesian. By induction, $W_{n-1}(B)$ is étale over $W_{n-1}(A)$, and so this follows from 9.1, which we can apply by 6.1 and 6.8 .

Step 2: $W_{n}(B)$ is étale over $W_{n}(A)$. We know from 8.1(b) that the kernel $I_{n}(A)$ of the map $\alpha_{n}: W_{n}(A) \rightarrow \bar{W}_{n}(A)$ has square zero. Therefore by [EGA 8, 18.1.2], there is an étale $W_{n}(A)$-algebra $C$ and an isomorphism $f: C \otimes_{W_{n}(A)} \bar{W}_{n}(A) \rightarrow \bar{W}_{n}(B)$. Now consider the square

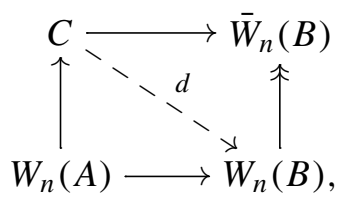

where the upper map is the one induced by $f$ and where $d$ will soon be defined. By 8.1(b), the kernel $I_{n}(B)$ of the right-hand map has square zero. Therefore since $C$ is étale over $W_{n}(A)$, there exists a unique map $d$ making the diagram commute. Let us now show that $d$ is an isomorphism. 
Because $C$ is étale and hence flat over $W_{n}(A)$, we have a commutative diagram with exact rows:

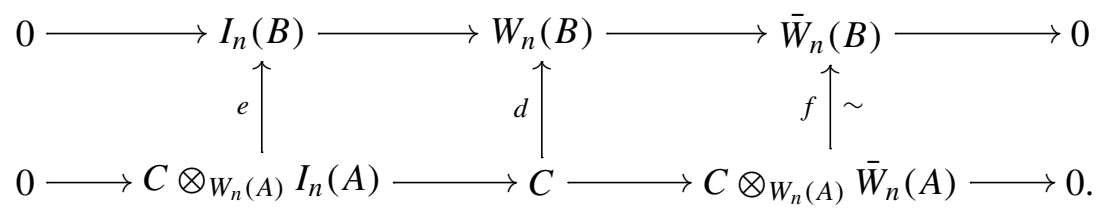

So to show $d$ is an isomorphism, it is enough to show $e$ is an isomorphism. Because $I_{n}(A)$ is a square-zero ideal, the action of $W_{n}(A)$ on it factors through $\bar{W}_{n}(A)$. Therefore, $e$ factors as follows:

$C \otimes_{W_{n}(A)} I_{n}(A)=C \otimes_{W_{n}(A)} \bar{W}_{n}(A) \otimes_{\bar{W}_{n}(A)} I_{n}(A)$

$$
\stackrel{f \otimes \mathrm{id}}{\longrightarrow} \bar{W}_{n}(B) \otimes_{\bar{W}_{n}(A)} I_{n}(A) \stackrel{g}{\longrightarrow} I_{n}(B),
$$

Since $f$ is an isomorphism, it is enough to show $g$ is an isomorphism.

Using the description (8-0-3) of $I_{n}$, the map $g$ can be extended to the following commutative diagram with exact rows:

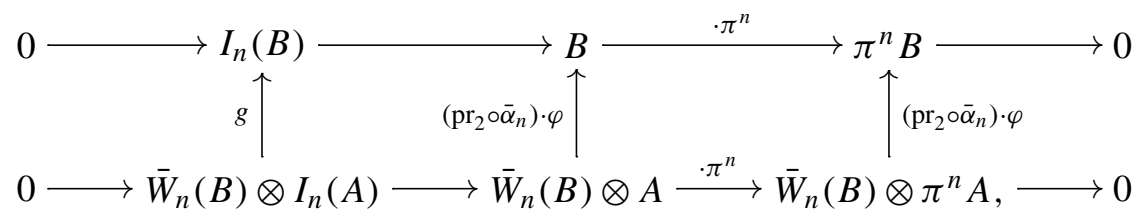

where $\otimes$ denotes $\otimes_{\bar{W}_{n}(A)}$, for short. Therefore it is enough to show the right two vertical maps are isomorphisms, and to do this, it is enough to show the right-hand square in the diagram

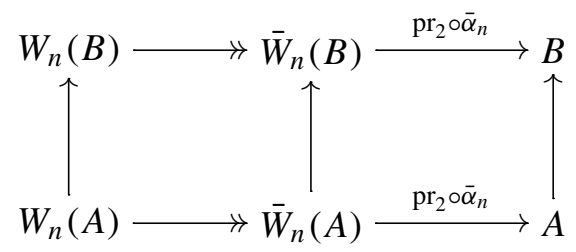

is cocartesian. We will do this by applying 9.1, with $U=\operatorname{Spec} R[1 / \mathfrak{m}] \otimes_{R} \bar{W}_{n}(A)$.

By step 1, condition 9.1(a) holds. Now consider conditions 9.1(b)-(c). By 8.3(b), the horizontal maps in the left-hand square have square-zero kernel. In particular, the scheme maps they induce are universal homeomorphisms. And by 6.1, they become isomorphisms after applying $R[1 / \mathfrak{m}] \otimes_{R}-$. Therefore it is enough to show 9.1(b)-(c) hold for the perimeter of the diagram above. In this case, 9.1(b) follows from 6.1, and 9.1(c) follows from 6.8. 
9.3. Remark. Observe that when $A$ is $E$-flat, the proof terminates after step 1, which is just an application of 7.12. Thus in the central case, the argument is not much more than 8.1 and some general descent theory.

9.4. Corollary. Let $B$ an étale A-algebra, and let $C$ be any A-algebra. Then for any $n \in \mathbb{N}^{(E)}$, the induced diagram

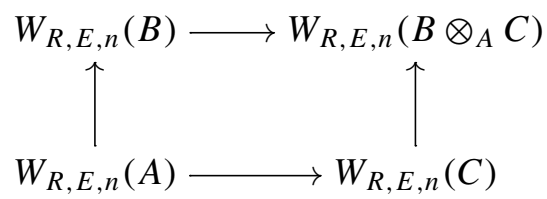

is cocartesian.

Proof. By 5.4, we can assume $E$ consists of a single ideal $\mathfrak{m}$. The proof will be completed by 9.1 , once we check its hypotheses are satisfied. Condition (a) of 9.1 holds by 9.2 , condition (b) holds by 6.1 and 2.7 , and condition (c) holds by 6.8 .

9.5. $W_{n}$ does not generally commute with coproducts. Almost anything is an example. For instance, with the $p$-typical Witt vectors, $W_{1}\left(A \otimes_{\mathbb{Z}} A\right)$ is not isomorphic to $W_{1}(A) \otimes_{W_{1}(\mathbb{Z})} W_{1}(A)$, when $A$ is $\mathbb{F}_{p}[x]$ or $\mathbb{Z}[x]$.

9.6. $W$ does not generally preserve étale maps. Let $W$ denote $p$-typical Witt functor (non-truncated), and let $\varphi$ denote the evident inclusion $\mathbb{Q}[x] \rightarrow \mathbb{Q}\left[x^{ \pm 1}\right]$, which is étale. While $W(\varphi)$ is best viewed as a map of pro-rings, it is possible to view it as a map of ordinary rings, and ask whether it is étale. It is not: $W(\varphi)$ can be identified with $\varphi^{\mathbb{N}}: \mathbb{Q}[x]^{\mathbb{N}} \rightarrow \mathbb{Q}\left[x^{ \pm 1}\right]^{\mathbb{N}}$, which is not étale because $\mathbb{Q}\left[x^{ \pm 1}\right]^{\mathbb{N}}$ is not finitely generated as an $\mathbb{Q}[x]^{\mathbb{N}}$-algebra. This is an elementary exercise.

9.7. Other truncation sets for the big Witt vectors. Some writers have considered more general systems of truncations for the big Witt functor 1.15. See [Hesselholt and Madsen 1997, §4.1], for example. Given a finite set $T$ of positive integers closed under extraction of divisors, they define an endofunctor $W_{T}$ of the category of rings. When $T$ consists of all the divisors of some integer $d \geqslant 1$, then $W_{T}$ agrees with our $W_{\mathbb{Z}, E, n}$, where $E$ consists of the maximal ideals $\mathfrak{m} \subset \mathbb{Z}$ that contain $d$ and where $n_{\mathfrak{m}}=\operatorname{ord}_{\mathfrak{m}}(d)$. Thus the two systems of truncations are cofinal with respect to each other.

The functors $W_{T}$ also preserve étale maps. Indeed, it is enough to show that the base change to $\mathbb{Z}[1 / T]$ and to $\mathbb{Z}_{(p)}$, for each prime $p \in T$, is étale. (See [EGA 8, 17.7.2(ii)].) Applying the identity $W_{T}(A)[1 / p]=W_{T}(A[1 / p])$, which can be established by looking at the graded pieces of the Verschiebung filtration, it is enough to consider $\mathbb{Z}[1 / T]$-algebras and $\mathbb{Z}_{(p)}$-algebras. In the either case, $W_{T}(A)$ is simply a product of $p$-typical Witt rings $W_{n}(A)$ for various primes $p$ and lengths 
$n$ (see [Hesselholt and Madsen 1997, (4.1.10)]), in which case the result follows from 9.2, or van der Kallen's original theorem [1986, (2.4)].

\section{Acknowledgements}

I thank Amnon Neeman for helpful discussions on some technical points and Lance Gurney for comments on earlier versions of this paper.

\section{References}

[Borceux 1994a] F. Borceux, Handbook of categorical algebra, I: Basic category theory, Encyclopedia of Mathematics and its Applications 50, Cambridge University Press, Cambridge, 1994. MR 96g:18001a Zbl 0803.18001

[Borceux 1994b] F. Borceux, Handbook of categorical algebra, II: Categories and structures, Encyclopedia of Mathematics and its Applications 51, Cambridge University Press, Cambridge, 1994. MR 96g:18001b Zbl 0843.18001

[Borger 2010] J. Borger, “The basic geometry of Witt vectors, II: spaces”, 2010. To appear in Math. Ann. arXiv 1006.0092

[Borger and Wieland 2005] J. Borger and B. Wieland, "Plethystic algebra", Adv. Math. 194:2 (2005), 246-283. MR 2006i:13044 Zbl 1098.13033

[Bourbaki 1983] N. Bourbaki, Algèbre commutative, chapitre 8: dimension; chapitre 9: anneaux locaux noethériens complets, Hermann, Paris, 1983. Reprinted Springer, Berlin, 2006. MR 86j:13001 Zbl 0579.13001

[Buium 1996] A. Buium, “Geometry of p-jets”, Duke Math. J. 82 (1996), 349-367. MR 97c:14029 Zbl 0882.14007

[Buium 2005] A. Buium, Arithmetic differential equations, Mathematical Surveys and Monographs 118, American Mathematical Society, Providence, RI, 2005. MR 2006k:14035 Zbl 1088.14001

[Buium and Simanca 2009] A. Buium and S. R. Simanca, "Arithmetic Laplacians", Adv. Math. 220:1 (2009), 246-277. MR 2009m:12008 Zbl 1172.14027

[Drinfeld 1976] V. G. Drinfeld, "Coverings of p-adic symmetric regions", Funkcional. Anal. $i$ Priložen. 10:2 (1976), 29-40. In Russian; translated in Funct. Anal. Appl. 10:2 (1976), 107-115. MR 54 \#10281 Zbl 0346.14010

[EGA 1] A. Grothendieck, "Éléments de géométrie algébrique, I: le langage des schémas", Inst. Hautes Études Sci. Publ. Math. 4 (1960), 1-228. MR 33 \#7330 Zbl 0118.36206

[EGA 6] A. Grothendieck, "Éléments de géométrie algébrique, IV: Étude locale des schémas et des morphismes de schémas (seconde partie)", Inst. Hautes Études Sci. Publ. Math. 24 (1965), 1-231. MR 33 \#7330 Zbl 0135.39701

[EGA 8] A. Grothendieck, "Éléments de géométrie algébrique, IV: étude locale des schémas et des morphismes de schémas (quatrième partie)", Inst. Hautes Études Sci. Publ. Math. 32 (1967), 1-361. MR 39 \#220 Zbl 0153.22301

[Grothendieck 1958] A. Grothendieck, "La théorie des classes de Chern", Bull. Soc. Math. France 86 (1958), 137-154. MR 22 \#6818 Zbl 0091.33201

[Grothendieck 1966] A. Grothendieck, "Technique de descente et théorèmes d'existence en géometrie algébrique, I: généralités; descente par morphismes fidèlement plats", exposé 190 in Séminaire Bourbaki, 1959/1960, Benjamin, New York, 1966. Reprinted as pp. 299-327 in Séminaire Bourbaki, vol. 5, Soc. Math. France, Paris, 1995. MR 1603475 Zbl 0229.14007 
[Hazewinkel 1978] M. Hazewinkel, Formal groups and applications, Pure and Applied Mathematics 78, Academic Press, New York, 1978. MR 82a:14020 Zbl 0454.14020

[Hesselholt and Madsen 1997] L. Hesselholt and I. Madsen, "Cyclic polytopes and the $K$-theory of truncated polynomial algebras", Invent. Math. 130 (1997), 73-97. MR 98k:19002 Zbl 0884.19004

[Joyal 1985a] A. Joyal, “ $\delta$-anneaux et $\lambda$-anneaux”, C. R. Math. Rep. Acad. Sci. Canada 7:4 (1985), 227-232. MR 86j:13024 Zbl 0583.13004

[Joyal 1985b] A. Joyal, " $\delta$-anneaux et vecteurs de Witt", C. R. Math. Rep. Acad. Sci. Canada 7:3 (1985), 177-182. MR 86j:13023 Zbl 0594.13023

[van der Kallen 1986] W. van der Kallen, "Descent for the $K$-theory of polynomial rings", Math. Z. 191:3 (1986), 405-415. MR 87h:13012 Zbl 0563.13011

[Lazard 1975] M. Lazard, Commutative formal groups, Lecture Notes in Mathematics 443, Springer, Berlin, 1975. MR 52 \#13861 Zbl 0304.14027

[Rydh 2009] D. Rydh, "Noetherian approximation of algebraic spaces and stacks", preprint, 2009. arXiv 0904.0227

[Rydh 2010] D. Rydh, "Submersions and effective descent of étale morphisms", Bull. Soc. Math. France 138:2 (2010), 181-230. MR 2679038 Zbl 05769982

[SGA 1] A. Grothendieck and M. Raynaud, Séminaire de Géométrie Algébrique du Bois Marie 1960/61: Revêtements étales et groupe fondamental (SGA 1), Lecture Notes in Mathematics 224, Springer, Berlin, 1971. Updated and annotated reprint, Soc. Math. de France, Paris, 2003. MR 50 \#7129 Zbl 0234.14002

[Tall and Wraith 1970] D. O. Tall and G. C. Wraith, "Representable functors and operations on rings”, Proc. London Math. Soc. (3) 20 (1970), 619-643. MR 42 \#258 Zbl 0226.13007

[Wilkerson 1982] C. Wilkerson, "Lambda-rings, binomial domains, and vector bundles over $\mathrm{CP}(\infty)$ ", Comm. Algebra 10:3 (1982), 311-328. MR 83f:55003 Zbl 0492.55004

[Witt 1937] E. Witt, “Zyklische Körper und Algebren der Charakteristik $p$ vom Grad $p^{n}$ : Struktur diskret bewerteter perfekter Körper mit vollkommenem Restklassenkörper der Charakteristik $p$ ”, $J$. Reine Angew. Math. 176 (1937), 126-140. Reprinted in [Witt 1998], 142-156. Zbl 0016.05101

[Witt 1998] E. Witt, Collected papers / Gesammelte Abhandlungen, edited by I. Kersten, Springer, Berlin, 1998. MR 99i:01038 Zbl 0917.01054

Communicated by Hendrik W. Lenstra

Received 2010-05-13 Revised 2010-08-08 Accepted 2010-10-13

james.borger@anu.edu.au Department of Mathematics, Australian National University, Mathematical Sciences Institute, Building 27, Canberra 2602, Australia http://wwwmaths.anu.edu.au/ borger/ 


\section{Algebra \& Number Theory}

www.jant.org

\section{EDITORS}

MANAGING EDITOR

Bjorn Poonen

Massachusetts Institute of Technology

Cambridge, USA

\author{
EDITORIAL BOARD CHAIR \\ David Eisenbud \\ University of California \\ Berkeley, USA
}

BOARD OF EDITORS

Georgia Benkart

Dave Benson

Richard E. Borcherds

John H. Coates

J-L. Colliot-Thélène

Brian D. Conrad

Hélène Esnault

Hubert Flenner

Edward Frenkel

Andrew Granville

Joseph Gubeladze

Ehud Hrushovski

Craig Huneke

Mikhail Kapranov

Yujiro Kawamata

János Kollár

Yuri Manin

Barry Mazur

Susan Montgomery
University of Wisconsin, Madison, USA

University of Aberdeen, Scotland

University of California, Berkeley, USA

University of Cambridge, UK

CNRS, Université Paris-Sud, France

University of Michigan, USA

Universität Duisburg-Essen, Germany

Ruhr-Universität, Germany

University of California, Berkeley, USA

Université de Montréal, Canada

San Francisco State University, USA

Hebrew University, Israel

University of Kansas, USA

Yale University, USA

University of Tokyo, Japan

Princeton University, USA

Northwestern University, USA

Harvard University, USA

University of Southern California, USA
Shigefumi Mori

Andrei Okounkov

Raman Parimala

Victor Reiner

Karl Rubin

Peter Sarnak

Michael Singer

Ronald Solomon

Vasudevan Srinivas

J. Toby Stafford

Bernd Sturmfels

Richard Taylor

Ravi Vakil

Michel van den Bergh

Marie-France Vignéras

Kei-Ichi Watanabe

Andrei Zelevinsky

Efim Zelmanov
RIMS, Kyoto University, Japan

Princeton University, USA

Emory University, USA

University of Minnesota, USA

University of California, Irvine, USA

Princeton University, USA

North Carolina State University, USA

Ohio State University, USA

Tata Inst. of Fund. Research, India

University of Michigan, USA

University of California, Berkeley, USA

Harvard University, USA

Stanford University, USA

Hasselt University, Belgium

Université Paris VII, France

Nihon University, Japan

Northeastern University, USA

University of California, San Diego, USA

\section{PRODUCTION}

contact@msp.org

Silvio Levy, Scientific Editor

Andrew Levy, Production Editor

See inside back cover or www.jant.org for submission instructions.

The subscription price for 2011 is US \$150/year for the electronic version, and \$210/year (+ \$35 shipping outside the US) for print and electronic. Subscriptions, requests for back issues from the last three years and changes of subscribers address should be sent to Mathematical Sciences Publishers, Department of Mathematics, University of California, Berkeley, CA 94720-3840, USA.

Algebra \& Number Theory (ISSN 1937-0652) at Mathematical Sciences Publishers, Department of Mathematics, University of California, Berkeley, CA 94720-3840 is published continuously online. Periodical rate postage paid at Berkeley, CA 94704, and additional mailing offices.

ANT peer review and production are managed by EditFLOw ${ }^{\mathrm{TM}}$ from Mathematical Sciences Publishers.

PUBLISHED BY

mathematical sciences publishers

http://msp.org/

A NON-PROFIT CORPORATION

Typeset in LATEX

Copyright $\odot 2011$ by Mathematical Sciences Publishers 


\section{Algebra \& Number Theory}

\section{Volume $5 \quad$ No. $2 \quad 2011$}

On the Hom-form of Grothendieck's birational anabelian conjecture in positive characteristic

\section{MOHAMED SAÏDI and AKIO TAMAGAWA}

Local positivity, multiplier ideals, and syzygies of abelian varieties

RoBERT LAZARSFELD, GIUSEPPE PARESCHI and MiHNEA POPA

KATHERINE STANGE

The basic geometry of Witt vectors, I The affine case

JAMES BORGER

Correction to a proof in the article Patching and admissibility over two-dimensional complete local domains

DANNY NEFTIN and ELAD PARAN 